\title{
Evaluation of Nutritional Adequacy and Symptom Improvement During Implementation of the Low-FODMAP Diet in Individuals with Irritable Bowel Syndrome
}

\section{THESIS}

Presented in Partial Fulfillment of the Requirements for the Degree Master of Science in the Graduate School of The Ohio State University

By

Julie Richards, B.S.

Graduate Program in Allied Medicine

\author{
The Ohio State University
}

2018

Master's Examination Committee:

Dr. Marcia Nahikian-Nelms, Advisor

Dr. Kristen Roberts

Dr. Jill Clutter 
Copyrighted by

Julie Richards

2018 


\begin{abstract}
Irritable bowel syndrome (IBS) is a common functional gastrointestinal disorder (FGID) that affects $10-20 \%$ of adults and adolescents worldwide. A connection has been identified between the consumption of some foods and an exacerbation of IBS symptoms. One nutrition therapy option for IBS is the low fermentable oligosaccharides, disaccharides, monosaccharides, and polyols (FODMAP) diet. A lack of data describing the mechanism of action of this dietary pattern has led to insufficient evidence to establish standard clinical guidelines regarding the use of the low-FODMAP diet in IBS. This study aims to define the duration of the low-FODMAP diet elimination phase for optimal symptom relief and the nutritional adequacy of the low-FODMAP diet.

Seventy patients with IBS attended low-FODMAP diet group education classes that were provided monthly, and those who consented to the study were instructed to follow the low-FODMAP diet for six weeks. Subjects completed an IBS-Symptom Severity Scale questionnaire (IBS-SSS) at baseline and at weeks 2, 4, and 6. Subjects completed a three-day diet record at baseline and week 2 . Dietary compliance was assessed through daily high-FODMAP food checklists.
\end{abstract}

A total of 35 participants enrolled in this study. Complete data was available for $14(40 \%)$ participants. Incomplete data was available for $2(6 \%)$ participants, and 19 (54\%) dropped out of the study. Forty-three percent of the original participants $(15 / 35)$ 
were responders to the diet (a reduction $\geq 50$ points in IBS-SSS), and $87 \%(13 / 15)$ of responders saw a clinical response after two weeks of the diet. There was a significant reduction in symptoms seen between baseline and week two $(\mathrm{p}<0.001)$. Post initiation of the low-FODMAP diet in those participants with complete data, there was a statistically significant reduction in grams of carbohydrates consumed $(p=0.031)$. Servings from grains and servings from dairy were also significantly reduced $(p=0.048, p=0.010)$. Carbohydrate intake was below the recommended range in $79 \%$ of participants $(<45 \%$ of energy intake). Dietary fat intake was above the recommended range ( $>35 \%$ of energy intake) in $64 \%$ of participants. Calcium, iron, folate, vitamin C, and vitamin D intake were below the recommended dietary allowance (RDA) for $>75 \%$ of participants, although a majority of participants were consuming less than the RDA for these micronutrients at baseline. For participants who returned data, 71\% reported noncompliance.

These findings support that a low-FODMAP diet elimination phase of two weeks was optimal for achieving symptom relief. The low-FODMAP diet may lead to a nutritionally inadequate dietary pattern and must be monitored in future studies. The high dropout rate and poor dietary compliance reported in this study highlight the need to assess dietary compliance in future interventions to determine if a reduction in IBS-SSS is a result of low dietary FODMAP ingestion or a placebo effect. This study proposes that a proper dietitian led education session can help maintain an adequate nutritional intake by educating patients on proper substitutions of FODMAP containing foods, and a group education session is suitable for this dietary intervention. More rigourous studies with a 
larger sample size are necessary to evaluate nutritional adequacy and compliance with the low-FODMAP diet. 


\section{Acknowledgments}

I would like to thank my advisor, Dr. Marcia Nahikian-Nelms, for the opportunity to work with her and for all the guidance and encouragement. I would also like to thank my committee members, Dr. Kristen Roberts and Dr. Jill Clutter, for their support, dedication, and the expertise they provided. The three of you have helped me to grow as a researcher and for that I am very thankful.

Many thanks to Kristen Heitman, MS, RDN, from the Clinical Research Center at The Ohio State University, for entering my dietary data and educating me on NDSR. Also, thanks to those at The Ohio State University Medical Center's Division of Gastroenterology, Hepatology, and Nutrition for helping to organize the low-FODMAP diet education classes.

I want to thank my family - my mom for all the inspiration, my dad for his soothing advice, my siblings for the constant support, and the rest of the Richards and Ankrom families who have been there for me no matter what.

Finally, I would like to thank my friends who have remained by my side and always give me a reason to take a break, regardless of if they live in Columbus or across the country. I would be remiss if I didn't specifically thank my roommate, Anne Lemenager, who has provided continuous reassurance throughout the past two years of graduate school. 


\section{Vita}

2012

High School - Circleville,

Ohio

2016.

B.S. Nutrition with a

Dietetics

Concentration, Kinesiology -

Miami University

2016 to present.

Graduate Combined Program

in

Medical Dietetics - The Ohio

State University

\section{Fields of Study}

Major Field: Allied Medicine 


\section{Table of Contents}

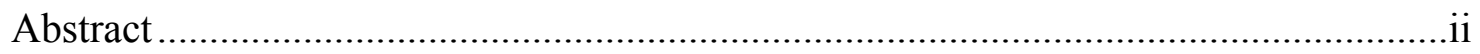

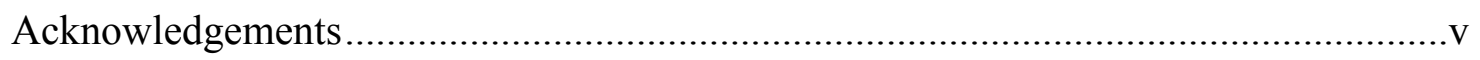

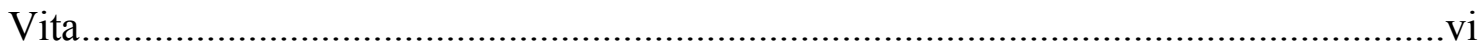

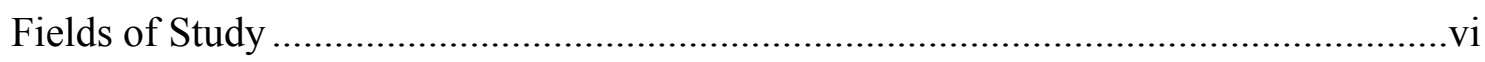

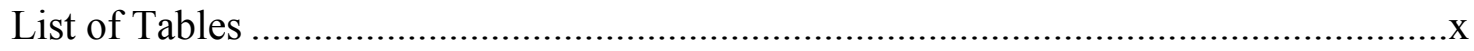

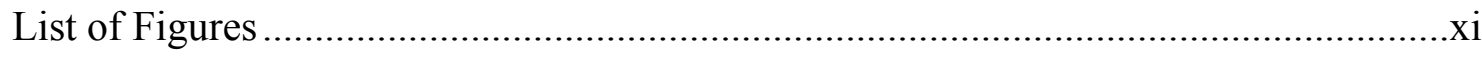

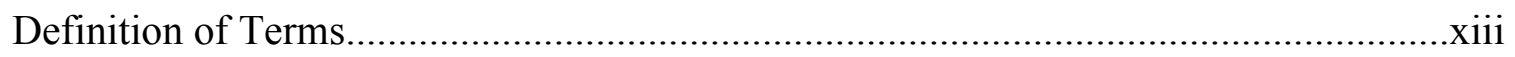

Chapter 1: Introduction ......................................................................................

Statement of the Problem.....................................................................................

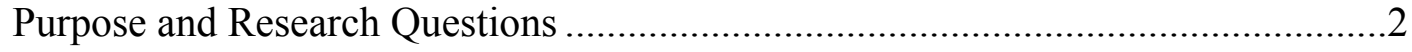

Theoretical Framework .............................................................................

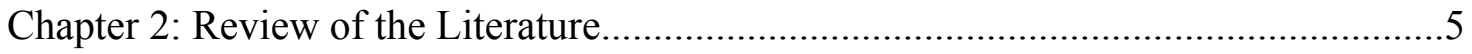

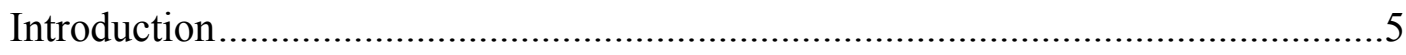

The Definition of Irritable Bowel Syndrome....................................................6

The Epidemiology and Economic Impact of IBS .........................................6

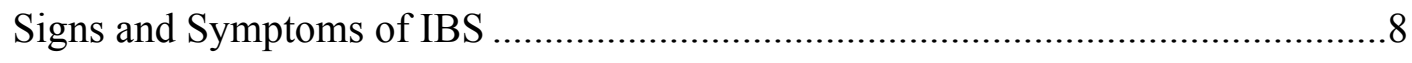

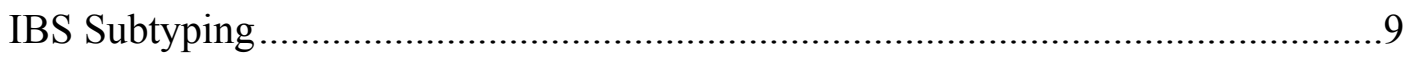

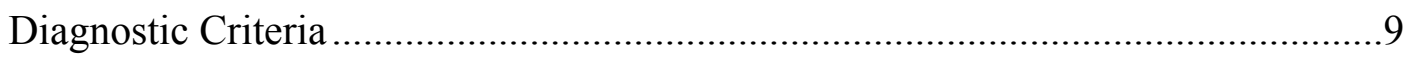

vii 


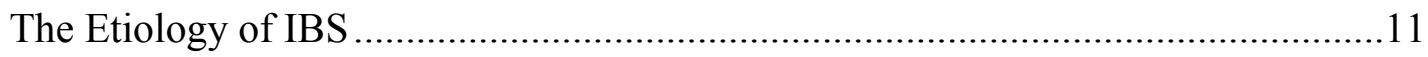

Pathogenesis and Pathophysiology of IBS ………………....................................11

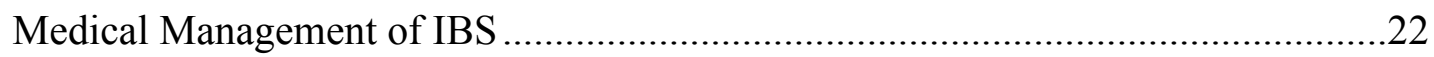

Complementary Health Approaches …………………………….........................22

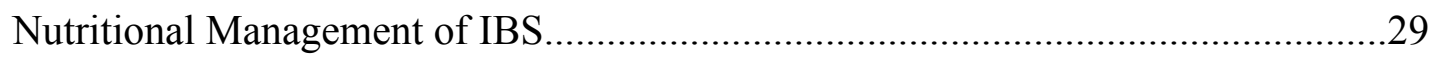

Effect of FODMAPs in the GI Tract and the Low-FODMAP Diet............................36

Limitations and Need for Further Research.............................................................49

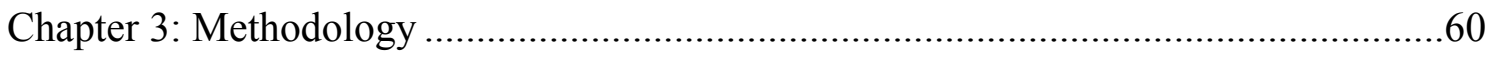

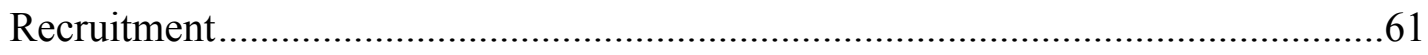

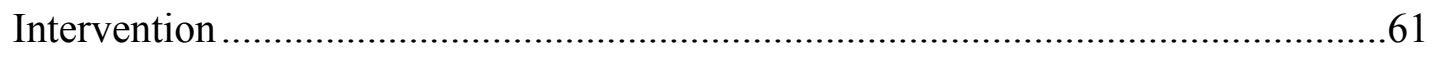

Additional Data Collection Sources........................................................................65

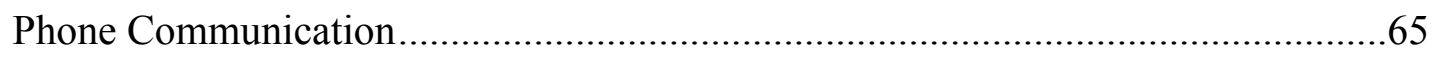

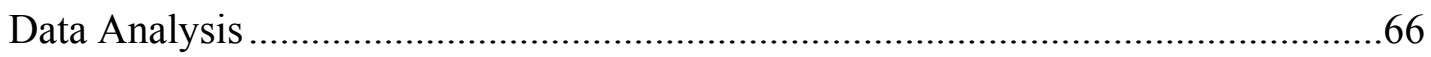

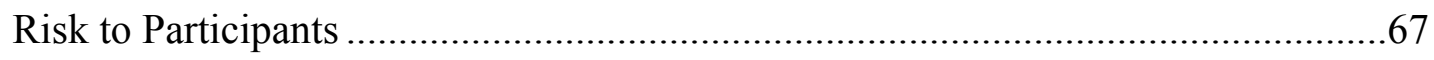

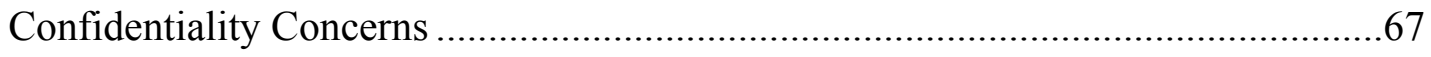

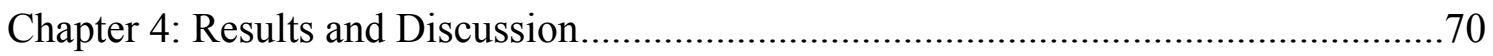

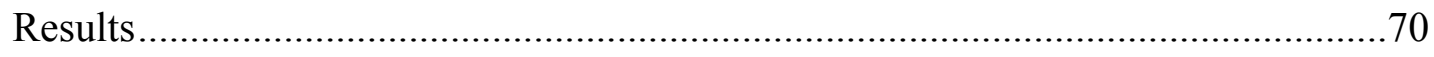

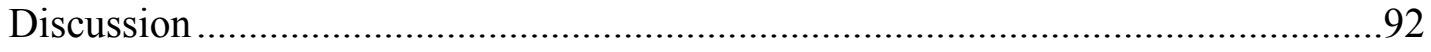

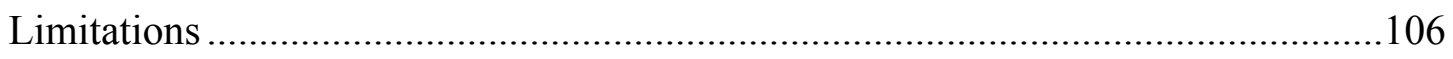

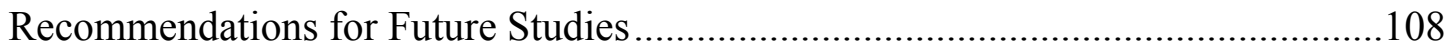

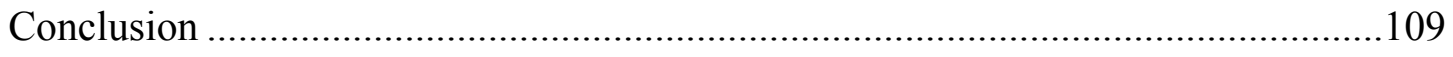

Chapter 5: Journal Article .................................................................................111

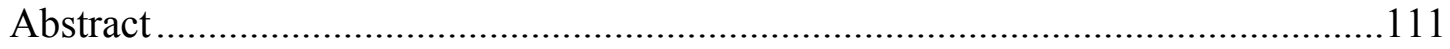

viii 


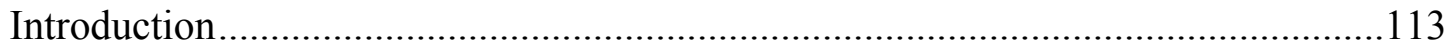

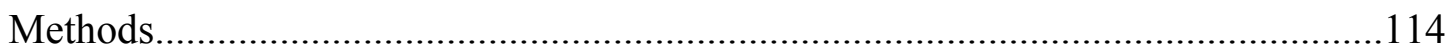

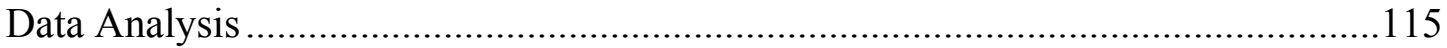

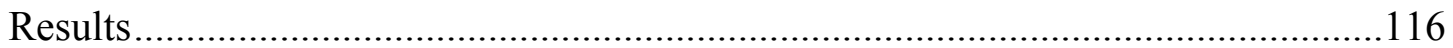

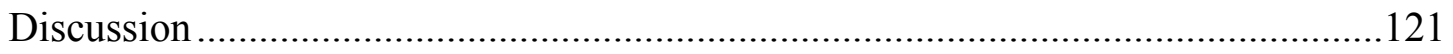

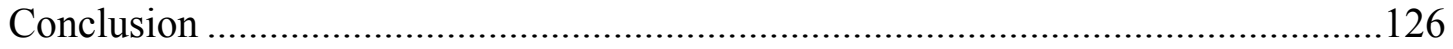

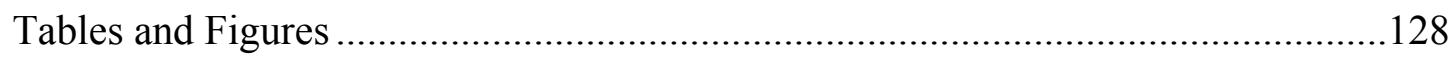

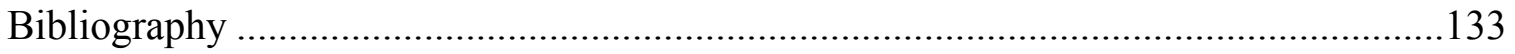

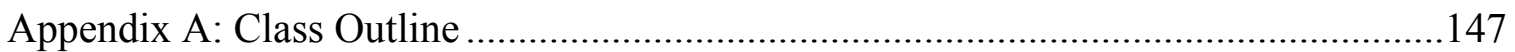

Appendix B: Demographics and Health Questionnaire ............................................159

Appendix C: IBS-Symptom Severity Score (IBS-SSS) and Bristol Stool Form Scale ...163

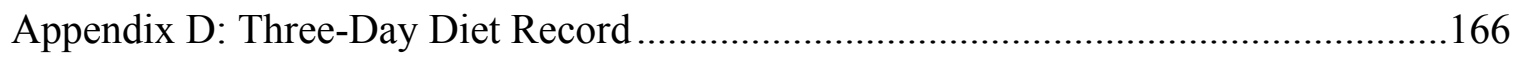

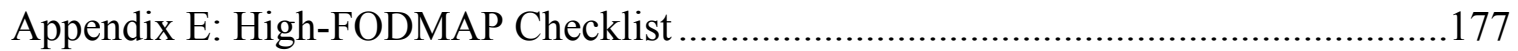

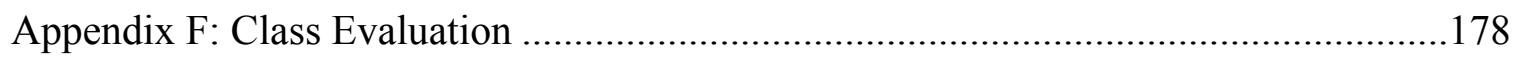




\section{List of Tables}

Table 1. Summary of Primary Research Studies on the Low-FODMAP Diet in Patients

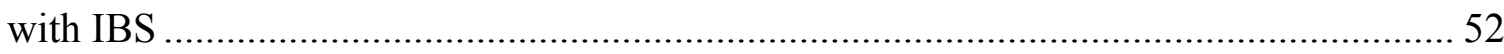

Table 2. Methods of Measuring Compliance in Low-FODMAP Diet Studies................ 59

Table 3. Summary of Study Instrumentation and Additional Data Collection Sources ... 68

Table 4. Demographic Information of Subjects with Irritable Bowel Syndrome............. 71

Table 5. Participant's Response and Compliance to the Low-FODMAP Diet................. 76

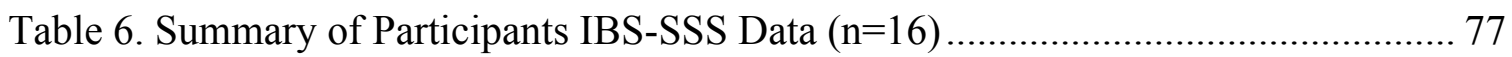

Table 7. Distribution of Participants Based on Percent Nutrient Intake Recommendations

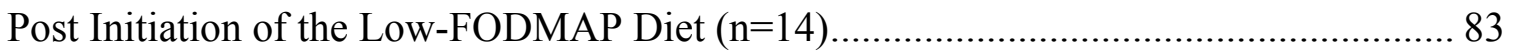

Table 8. Summary of Changes in Nutrient Intake from Baseline to Week Two of the

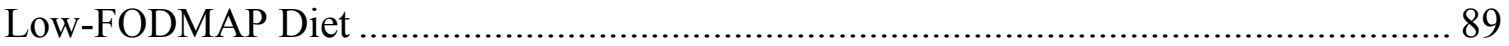

Table 9. Demographic and Health History Results for Patients with Irritable Bowel

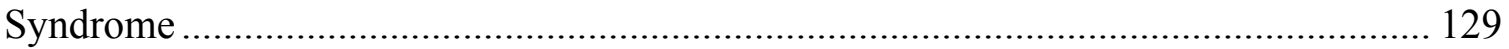

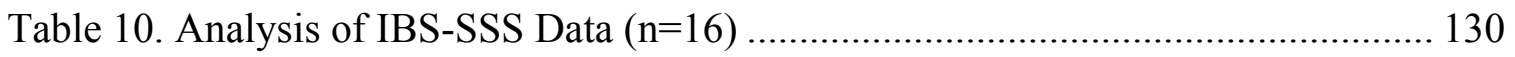

Table 11. Analysis of Nutrient Intake Post Low-FODMAP Diet Initiation $(n=14) \ldots \ldots . .130$

Table 12. Distribution of Participants Meeting Nutrient Recommendations Post Low-

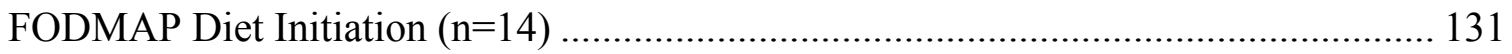




\section{List of Figures}

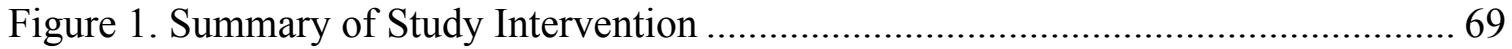

Figure 2. Low-FODMAP Diet Education Class Attendance and Study Enrollment........ 70

Figure 3. FODMAP Food Groups Restricted Prior to Low-FODMAP Education............ 73

Figure 4. Prescription Treatments Utilized by All Patients with IBS (\%) $(\mathrm{n}=35) \ldots \ldots \ldots \ldots . .73$

Figure 5. Non-Prescription Treatments Utilized by All Patients with IBS (\%) $(\mathrm{n}=35) \ldots .74$

Figure 6. IBS Diagnosis Based on Subtype According to Bristol Stool Form Scale

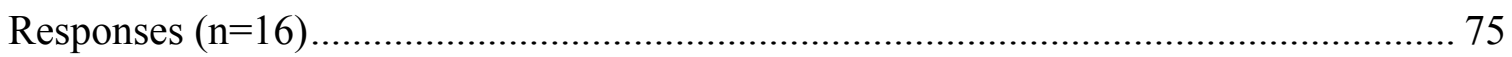

Figure 7. Frequency of High-FODMAP Foods Consumed by Non-Compliant Participants

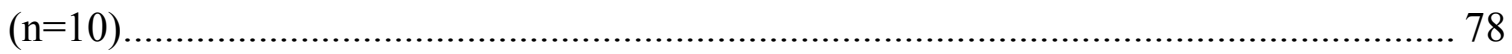

Figure 8. Pre- and Post-Diet Mean Caloric Intake for All Participants(n=14) ................. 79

Figure 9. Pre- and Post-Diet Macronutrient Intake for All Participants $(\mathrm{n}=14) \ldots \ldots \ldots \ldots . . . .80$

Figure 10. Pre- and Post-Diet Mean Distribution of Energy Intake for All Participants

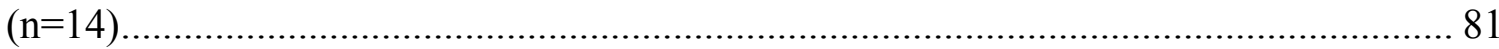

Figure 11. Mean Change in Fiber Intake After Initiation of the Low-FODMAP Diet for

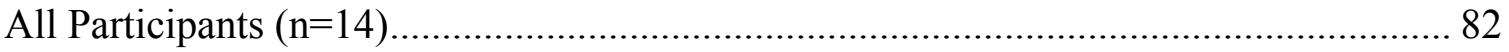

Figure 12. Mean Change in Vitamin C Intake After Initiation of the Low-FODMAP Diet

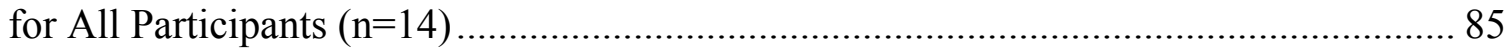


Figure 13. Mean Change in Vitamin D Intake After Initiation of the Low-FODMAP Diet

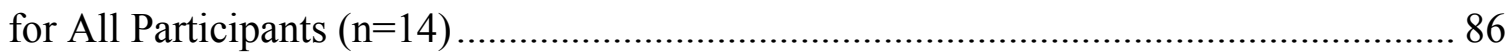

Figure 14. Mean Change in Folate Intake After Initiation of the Low-FODMAP Diet for

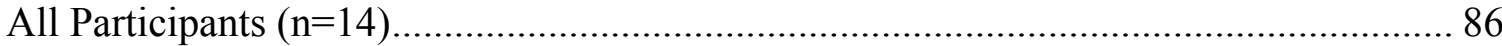

Figure 15. Mean Change in Calcium Intake After Initiation of the Low-FODMAP Diet for All Participants $(\mathrm{n}=14)$ 87

Figure 16. Mean Change in Iron Intake After Initiation of the Low-FODMAP Diet for All

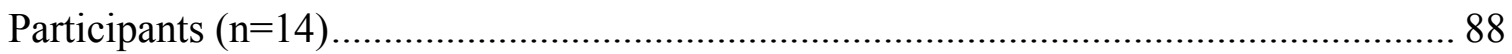

Figure 17. Mean Change in Servings of Food Groups Post-Diet Initiation ( $n=14) \ldots \ldots \ldots . .89$

Figure 18. Patients' Preferred Type of Dietary Education Session $(n=45)$................... 91

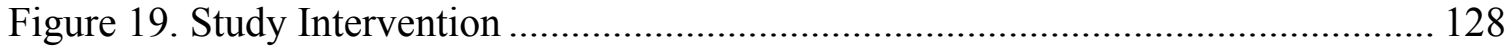

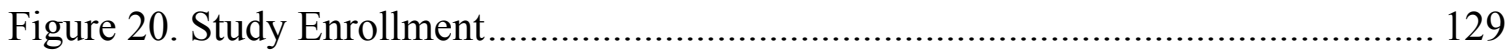

Figure 21. Mean Change in Food Group Servings $(n=14)$.......................................... 132

Figure 22. Preferred Dietary Education Session Feedback from Class Evaluation....... 132 


\section{Definition of Terms}

\section{Conceptual definitions for this Study}

1. FGID - Functional gastrointestional disorder; a disorder that affects different parts of the intestinal tract and involves visceral hypersensitivity and impaired motility. ${ }^{11}$

2. IBS - Irritable bowel syndrome; an FGID characterized by abdominal pain and abnormal changes in bowel habits. ${ }^{11}$

3. FODMAP - Acronym for fermentable oligosaccharides, disaccharides, monosaccharides, and polyols; a group of poorly digested carbohydrates. ${ }^{11}$

4. Oligosaccharide - A carbohydrate compound that is made of a small number of monosaccharides. ${ }^{11}$

5. Disaccharide - A carbohydrate compound that is a condensation product of two monosaccharides by the elimination of water. ${ }^{11}$ Examples are sucrose, lactose, and maltose.

6. Monosaccharide - The simplest form of a carbohydrate, which cannot be broken down further by hydrolysis. ${ }^{11}$ Examples are glucose, fructose, and galactose.

7. Polyol - Short for polyhydroxy alcohol; a sugar containing multiple $-\mathrm{OH}$ groups. Examples are sugar alcohols and inositols. ${ }^{11}$ 
8. Prebiotic - Food components that are non-digestible; therapeutic nutrition preparation that is used for favoring the growth of beneficial bacteria in the GI tract and not favoring the growth of pathogenic organisms. ${ }^{11}$

9. Probiotic - A live bacteria or yeast that supplements beneficial bacterial flora. ${ }^{11}$

10. RDA - Recommended dietary allowance; the number of total calories and specific nutrients necessary per day to maintain good health. ${ }^{11}$

11. AMDR - Acceptable Macronutrient Distribution Range; the range of energy intake for a particular energy source set by the Institute of Medicine. ${ }^{11}$

12. HBT - Hydrogen breath test; can be used as a diagnostic test for small intestinal bowel overgrowth or carbohydrate malabsorption (fructose, lactose, sorbitol). ${ }^{11}$

13. NDSR - The Nutrition Data System for Research Software; developed by the Nutrition Coordinating Center (NCC) at the University of Minnesota, in Minneapolis, MN to analyze nutritional data.

\section{Operational Definitions for this Study}

1. Elimination phase - First phase of the low-FODMAP diet; period where patients following the low-FODMAP diet completely restrict high-FODMAP foods from their daily intake.

2. Reintroduction phase - Second phase of the low-FODMAP diet; period where patients following the low-FODMAP diet add one food item per week from a single FODMAP group to determine tolerance.

3. Personalization phase - Final phase of the low-FODMAP diet; period where patients individualize the low-FODMAP diet to maintain symptom control while promoting variety and nutritional adequacy. 
4. High-FODMAP food - a food containing one or more FODMAPs.

5. Low-FODMAP food - a food containing little to no FODMAPs. 


\section{Chapter 1: Introduction}

Irritable bowel syndrome (IBS) is classified as a Functional Gastrointestinal Disorder (FGID) and affects $10-20 \%$ of adults and adolescents worldwide. ${ }^{1}$ IBS is a complex disorder that is multifactorial, but a connection between exacerbation of symptoms and the ingestion of food has been determined through the literature. ${ }^{2-3}$ There is unknown etiology and questionable pathophysiology making it difficult to treat IBS symptoms. Furthermore, a concrete dietary approach to alleviate IBS symptoms has not

yet been established. ${ }^{4}$ Medical management of symptoms relies heavily on medications, although many of these are unsupported by sufficient evidence. ${ }^{5}$ The low-FODMAP diet has gained attention as an effective approach for managing IBS symptoms. As a novel therapeutic approach, further research is necessary to sculpt the low-FODMAP diet into a strong, evidence-based dietary option for symptom relief in IBS. ${ }^{6}$ The diet's highly restrictive nature heightens concern regarding the nutritional adequacy and emphasizes a need for better understanding the minimum required length of the diet's elimination phase.

\section{Statement of the Problem}

Irritable bowel syndrome is characterized by lower abdominal pain or discomfort associated with a change in stool frequency or consistency. ${ }^{7}$ The ingestion of food has 
been widely documented in the literature and by patients to be a major contribution to IBS symptoms. A dietary restriction of fermentable carbohydrates has shown to be effective in managing symptoms of IBS. ${ }^{8}$ This diet is known as the low fermentable oligosaccharides, disaccharides, monosaccharides, and polyols (FODMAP) diet. This dietary approach is divided into three parts - an elimination phase, a reintroduction phase, and FODMAP personalization phase. ${ }^{9}$ The elimination phase typically lasts for two to eight weeks, ${ }^{10}$ and all high-FODMAP dietary choices are restricted during this time. In the reintroduction phase, there are staged, dosed FODMAP challenges designed to assess tolerance. ${ }^{9}$ The FODMAP personalization phase aims to improve diet variety and nutritional adequacy over time while maintaining symptom control. ${ }^{9}$ The lowFODMAP diet is highly restrictive of fruit, vegetables, dairy, and grains, particularly during the elimination phase. Such a restrictive diet for an extended period raises concern for nutritional deficiencies. This pilot study evaluated the nutritional adequacy of the low-FODMAP diet elimination phase and the necessary duration of the elimination phase for optimal symptom improvement.

\section{Purpose and Research Questions}

The purpose of this study was to evaluate nutritional adequacy of the lowFODMAP diet and speed of symptom improvement to develop the optimal dietary intervention with the least restrictive protocol. This can help determine appropriate guidelines for the low-FODMAP diet to improve recommendations for the IBS-patient population. This study utilized existing materials and research on the low-FODMAP diet to instruct participants and observe outcomes related to the nutritional intake and speed of 
symptom improvement. There is currently insufficient evidence to establish firm clinical guidelines regarding the use of the low-FODMAP diet in the IBS patient population. Therefore, this study aims to further determine if a low-FODMAP diet can be proven as a successful therapeutic treatment for IBS. This study strives to offer insight into these current gaps in the literature with the following questions:

1. Are there differences in nutritional intake before and after initiation of the lowFODMAP diet?

2. When compared to reference standards, is nutrient intake adequate after two weeks of the low-FODMAP diet elimination phase?

3. What is the optimal duration for the elimination phase of the low-FODMAP diet to promote symptom relief?

\section{Theoretical Framework}

The theory of adult learning guided the framework for designing this study's intervention. The intervention of this study involved a nutrition education class where study participants learned the rationale and steps toward initiation of the low-FODMAP diet. Andragogy, the teaching of adult learners, explains that adult learning is problem centered, self-directed, and internally motivated. ${ }^{12}$ The problem addressed through the participation in this study is the symptoms of IBS, which can greatly affect an individual's health related quality of life (HRQOL). Many patients with IBS do not seek care from a professional to help alleviate symptoms and typically remain clinically undiagnosed. ${ }^{13,14,15}$ While this study's participants were all clinically diagnosed with IBS, participation in this study after attending the educational class was not mandatory. 
Therefore, the individuals who participated in this study displayed a desire to improve their IBS symptoms and increase their overall well-being. This demonstrated participants were internally motivated.

The andragogy approach to teaching focuses on being self-directed, meaning that adults take control to advance their knowledge in areas where learning is needed. ${ }^{12}$ This study's low-FODMAP education class outline was compatible with andragogy, as it involved a lecture followed by problem-solving activities. Participants were provided with resources written by Kate Scarlata, RDN, LD that they could use on their own to further enhance and develop their knowledge of the diet. The group class approach for learning the diet was meant to enhance positive attitudes toward resolution of the condition, and the class provided positive message stimuli through the emphasis of appropriate dietary choices for the low-FODMAP diet. 


\section{Chapter 2: Review of the Literature}

\section{Introduction}

A functional gastrointestinal disorder (FGID) explains a combination of chronic or recurrent gastrointestinal (GI) symptoms that cannot be explained by either structural or biochemical abnormalities. ${ }^{16}$ These disorders ultimately stem from disturbances in motor, secretory, or sensory GI function. Due to the non-structural nature, FGIDs are defined by symptoms. ${ }^{16}$ IBS is a common FGID characterized by abdominal pain and altered bowel habits. ${ }^{1,5,17}$ IBS is highly prevalent both nationally and globally, ${ }^{13,18,19}$ and results in a significant economic burden ${ }^{15}$ and undesirable impact on health related quality of life (HRQOL). ${ }^{20}$ The etiology of IBS is not well-defined due to an absence of

demonstrable pathology in IBS. ${ }^{21}$ There are numerous treatment options utilized for the management of IBS symptoms, including medical, dietary, and complementary and integrative health measures. However, many of these approaches are not supported by high-quality evidence. An increasing amount of research has supported the low fermentable oligosaccharide, disaccharide, monosaccharide, and polyol (FODMAP) diet as a treatment to help alleviate symptoms in IBS patients. ${ }^{6}$ This literature review explores the complex disorder of IBS, its prevalence, pathology and pathophysiology, diagnostic criteria, treatment options, nutrition therapies, and possible dietary triggers. Current 
research on the low-FODMAP diet is reviewed to increase understanding and identify areas for future research.

\section{The Definition of Irritable Bowel Syndrome}

IBS is an FGID that combines a proposed alteration in gut physiology with an interaction of psychosocial factors. ${ }^{22}$ Patients with IBS do not have structural or biochemical abnormalities. ${ }^{17}$ Therefore, IBS is defined as an illness rather than a disease. An illness relates to an individual's personal experiences related to medical condition, while a disease displays abnormalities in the body's structure or function. ${ }^{23}$ In IBS, there is a presence of abdominal pain or discomfort associated with altered bowel habits. ${ }^{1,5,17}$ IBS is classified by abdominal pain and changes in bowel habits lasting for a period of at least three months. ${ }^{17}$

\section{The Epidemiology and Economic Impact of IBS}

IBS affects approximately $11 \%$ of individuals globally. ${ }^{18}$ Differences in study methodology, diagnostic criteria, and cultural differences in symptom perception and reporting pattern can affect the actual population prevalence rate. ${ }^{24}$ Furthermore, it is difficult to fully determine the prevalence of IBS worldwide. The reviews by Lovell and Ford ${ }^{18}$ and Sperber et al. ${ }^{24}$ found heterogeneity between studies in different geographic regions, making it clear that a pooled prevalence rate is not a reliable statistic.

More specifically, IBS has been reported to affect around $11.8 \%$ of individuals in North America ${ }^{18}$ and is the most common diagnosis made by gastroenterologists in the United States. ${ }^{19}$ IBS requires management from gastroenterologists and/or Primary Care Physicians (PCPs) ${ }^{17}$ Hungin et al. ${ }^{13}$ estimated prevalence of IBS and symptom pattern 
through random-digit telephone dialing screenings. They discovered that $14.1 \%$ of people interviewed met IBS criteria. ${ }^{13}$ Prevalence of IBS has been found to decrease with increasing age and is more common in women than men. ${ }^{14,18}$ Specifically, women are 1.5 times more likely to develop IBS than men. ${ }^{17}$ The overall prevalence of IBS is 67 percent higher in women versus men internationally. ${ }^{14}$

Since no curative therapy currently exists for IBS, there is considerable economic impact. It has been found that IBS is associated with high direct costs as well as loss of productivity. In the United States, the mean direct cost of IBS per patient is $\$ 619$ annually, and total annual direct costs related to IBS are $\$ 1.35$ billion. ${ }^{15}$ Patients with IBS consume over 50\% more health care resources when compared to matched controls without IBS. ${ }^{17}$ Work productivity is significantly affected in patients with IBS. ${ }^{13}$ The average number of missed work days for patients with IBS was found to be 6.1 days compared to 3 days for individuals who do not suffer from IBS. ${ }^{13}$ Total estimated productivity costs related to an IBS diagnosis in the United States is an annual cost of $\$ 205$ million. $^{15}$

IBS affects patients' HRQOL. It has been found that HRQOL is decreased in patients with moderate to severe IBS, and the HRQOL of patients with IBS is comparable to other chronic diseases such as depression and GERD ${ }^{20}$ A patient's HRQOL is not significantly affected by which subtype of IBS a patient is experiencing, ${ }^{20}$ but attitudes toward HRQOL appear to be more affected for individuals who receive a definitive IBS diagnosis. ${ }^{13}$ The recent study by Ballou and $\mathrm{Keefer}^{25}$ found there is a high level of daily impairment and avoidance of daily activities for patients with IBS. An increased level of 
impairment was more common in patients who met criteria for depression, anxiety, and/or panic disorder. ${ }^{25}$

\section{Signs and Symptoms of IBS}

Most individuals diagnosed with IBS report lower abdominal pain or discomfort associated with a change in stool frequency or consistency. ${ }^{7}$ Other symptoms reported by patients are the passage of mucus through the rectum, abdominal pain relieved by defecation, and visible abdominal distension or the symptoms of bloating, flatulence, straining, and a sense of defecation urgency. ${ }^{7,21}$ Gas production after the ingestion of food and impaired gas transport may explain the mechanics behind bloating and distension in IBS patients, but the mechanism still remains somewhat unclear. $^{26}$ Females report a higher variety of intestinal and non-intestinal symptoms despite similar levels in men of IBS severity, psychological symptoms, and impact from illness. ${ }^{27}$

Symptoms can occur for many years with an average duration of 10 or more years. ${ }^{13}$ However, symptoms of IBS may not be consistent. Symptoms can come and go over time, ${ }^{1}$ and subtypes of IBS may change. ${ }^{5}$ Psychological stress can exacerbate GI symptoms in patients and affect GI function. For IBS patients who also are experiencing psychological stress, more severe symptoms may result. The psychological factors that a patient is experiencing could also alter symptom perception and a patient's reactions to

symptoms, ${ }^{1}$ and it may lower the pain threshold for patients. ${ }^{23}$ Alternatively, the presence of an FGID such as IBS may result in psychosocial consequences and affect an individual's well-being. ${ }^{22}$ 


\section{IBS Subtyping}

There are four subtypes of IBS: IBS with constipation (IBS-C), IBS with diarrhea (IBS-D), mixed type (IBS-M), and unclassified (IBS-U). ${ }^{5}$ The different subtypes of IBS have distinguishing signs and symptoms. Subtyping in IBS should be based on stool form using the Bristol Stool Form Scale. ${ }^{1}$ IBS-C is identified by hard or lumpy stools $\geq 25 \%$ of the time, and loose or watery stools $<25 \%$ of the time. ${ }^{1}$ IBS-D is identified by loose or watery stools $\geq 25 \%$ of the time and hard or lumpy stools $<25 \%$ of the time. ${ }^{1}$ IBS-M occurs when there are hard or lumpy stools $\geq 25 \%$ of the time and also loose or watery stools $\geq 25 \%$ of the time. ${ }^{1}$ The classification of IBS-U happens when there is an abnormality in stool consistency that does not meet criteria for any of the three other IBS subtypes. ${ }^{1}$ Post-infectious IBS (PI-IBS) can also occur for some individuals following cases of infective gastroenteritis. ${ }^{28}$ PI-IBS differs from other IBS subtypes by having more diarrheal features. ${ }^{28}$

\section{Diagnostic Criteria}

IBS is diagnosed clinically since there are no available IBS biomarkers. ${ }^{14}$ Biomarkers that have been evaluated for diagnosing IBS include visceral hypersensitivity, altered pain perception, serum biomarkers, fecal biomarkers, colonic mucosal biomarkers, and tight junction disruption. ${ }^{29}$ These diagnostic biomarkers do not perform any better than symptom based approaches. ${ }^{29}$ The first symptom based diagnostic criteria was the Manning criteria. ${ }^{29}$ The Manning criteria was based on responses to a questionnaire that identified 15 symptoms common in IBS. ${ }^{30}$ The current gold standard for diagnosis of IBS is the Rome criteria, ${ }^{7}$ which is more restrictive than 
the Manning criteria. ${ }^{14}$ The Rome III classification system separates FGIDs into distinct conditions for enhanced diagnosis and treatment. There are 23 adult and 17 pediatric FGIDs, and these FGIDs are separated into six major categories. IBS is classified as a functional bowel disorder, which is category C in the Rome III Classification System. ${ }^{22}$ Under category C, IBS is defined as pain associated with changes in bowel habits, whereas functional diarrhea is classified as no pain with loose stools and functional constipation is classified as no changes in bowel habit. ${ }^{22}$ Rome III Criteria states that to be classified as IBS, recurrent abdominal pain or discomfort should last three days per month for at least three months. This pain should be accompanied by two of the following: improvement with defecation, onset related to a change in stool frequency, or onset related to a change in the appearance of stool. ${ }^{5}$ Rome III Criteria also separates IBS into the four different subtypes: IBS-C, IBS-D, IBS-M, and IBS-U. ${ }^{5}$ The Bristol Stool Form Scale can be used to define bowel type. ${ }^{5}$ Rome IV Criteria was published in May of 2016 with two major changes to the IBS diagnostic criteria. ${ }^{31}$ One change was that "discomfort" was removed from the definition because this term was imprecise and has different meanings in every language. The other change was that abdominal pain should be present at least one day per week for three months, versus Rome III criteria where pain was only necessary for three days per month for three months. ${ }^{31}$ The Bristol Stool Form Scale developed by Lewis and Heaton ${ }^{32}$ classifies stool into seven different categories based on the form of the stool and can be used in clinical practice or research to better understand GI function. ${ }^{32}$

The IBS-Symptom Severity Score (IBS-SSS) developed by Francis and Whorwel ${ }^{33}$ evaluates individuals using a maximum achievable score of 500, and 
classifies participants as mild $(75-<175)$, moderate $(175-<300)$, or severe $(>300)$

based on the score. ${ }^{33}$ This assessment tool incorporates pain, distension, bowel

dysfunction, and quality of life. ${ }^{33}$ The IBS-SSS can be used as an instrument to assess changes in symptoms and classify the severity of IBS for a patient.

\section{The Etiology of IBS}

There is an absence of demonstrable pathology in IBS, but a combination of factors has been identified as potential contributors to the disorder. ${ }^{21}$ Therefore, the etiology of IBS is not well defined due to the complexity of the disease and its' functional nature.

\section{Pathogenesis and Pathophysiology of IBS}

Many hypotheses are proposed in the literature regarding the possible underlying mechanism of IBS. Dysmotility, visceral hypersensitivity, mucosal immune dysregulation, hormonal events, alterations of bacterial flora, post-infectious sequelae, and a disturbance in CNS-ENS interaction are questioned to contribute to the pathogenesis of IBS. ${ }^{1,22}$ Although the exact cause of IBS remains unclear, continued research has provided an increased understanding. There are three main hypotheses behind the integrated explanatory model of IBS for clinical practice: altered peripheral regulation of gut function, altered brain gut signaling, and psychological distress. ${ }^{34}$

\section{Altered Peripheral Regulation of Gut Function}

An altered peripheral regulation of gut function is triggered by changes in neurotransmitter signaling, GI inflammation, and GI microbial changes. ${ }^{34}$ Specific peripheral mechanisms can result in symptoms of IBS. These mechanisms include: 
abnormal colonic transit and rectal evacuation, intraluminal irritants, inflammation, altered bile acid synthesis, and alterations in the GI microbiome.

\section{Serotonin $(5-H T)$}

Serotonin (5-HT) is a main signaling molecule in the GI tract, and it is estimated that about $95 \%$ of the body's serotonin is in the GI tract. ${ }^{35-36}$ Serotonin plays a role in bowel physiology as an enteric neurotransmitter, and is a signaling molecule for mucosal sensory stimuli. ${ }^{36}$ Serotonin also functions in the brain-gut axis and a dysregulation of this axis may result in GI symptoms. ${ }^{36}$ There is evidence that a gene polymorphism in IBS patients is within the serotonin transporter gene. More specifically, research demonstrates that a serotonin transporter polymorphism may be important in determining what IBS subtype. ${ }^{37}$ Bearcroft et al. ${ }^{38}$ found 5-HT concentrations were higher in IBS-D patients during a four-hour postprandial period, and the duration of the 5-HT peak was significantly longer in IBS-D patients. These results suggest that there may be a difference in the way 5-HT is released in IBS-D patients, and there could be a role for serotonin in the cause of IBS symptoms. ${ }^{38}$

Mast cells could have an important role in the serotonin signaling for IBS patients. ${ }^{35}$ When enteroendocrine cells or variable antigens secrete serotonin, mast cells in the lamina propria can be activated. When the mast cells are activated, they induce processes involving serotonin-mast-cell-neuroimmune mechanisms, and this process could contribute to the symptoms of pain and stool changes. ${ }^{35}$ Cremon et al. ${ }^{39}$ evaluated 5-HT and mast cell mediators and their relation to the IBS symptom of abdominal pain. It was found that the spontaneous release of 5-HT was increased in IBS patients compared with controls and this correlated with mast cell concentration and severity of abdominal 
pain, suggesting that the release of 5-HT contributes to the symptom of abdominal pain. The strong correlation between 5-HT released and the area of lamina propria occupied by mast cells suggests that mast cell infiltration could drive 5-HT release. ${ }^{39}$

\section{$>$ Endocrine Cells}

Abnormalities in endocrine cells could also be a factor in the etiology of IBS. The GI tract contains at least 15 different endocrine cells that are spread throughout the epithelial cells of the mucosa. These cells regulate sensitivity, motility, secretion, absorption, local immune defense, and food intake. ${ }^{40}$ Genetic factors, diet, the GI microbiota, and mucosal low-grade inflammation are all factors suggested to play a role in the development of IBS, and these factors can affect the GI endocrine cell concentration. $^{40}$

Abnormal endocrine cell densities are seen in both IBS and PI-IBS patients. Typically, endocrine cell densities are decreased in IBS patients, but in PI-IBS patients the density of endocrine cells increases. ${ }^{40}$ El-Salhy et al. ${ }^{41}$ discovered abnormal small intestine endocrine concentrations in IBS-patients, specifically in IBS-D patients. A later study by El-Salhy et al. ${ }^{42}$ also found abnormalities in the rectal endocrine cell densities for both IBS-D and IBS-C patients. The abnormal density of endocrine cells in IBS patients could be responsible for the visceral hypersensitivity, dysmotility, and abnormal gut secretion seen in IBS patients. ${ }^{40}$ Moreover, endocrine cell abnormalities may potentially cause some of the symptoms seen in IBS patients. ${ }^{40}$ Chromogranin A (CgA) is a common marker for duodenal endocrine cells and is typically reduced in IBS patients. CgA could be used as a sufficient biomarker for the diagnosis of IBS in all subgroups. ${ }^{43}$ 


\section{Altered Gastrointestinal Motility}

Minor alterations in GI motility are common in the IBS population. ${ }^{37}$ It is unclear if dysmotility is related to IBS symptoms, as it is probably more important in understanding bowel habits for certain IBS subgroups. ${ }^{37}$ The increased psychological stress seen in IBS patients can be related to dysmotility. ${ }^{23}$ Delayed gastric emptying has also been proposed as a factor in IBS, but research is conflicting. A relationship between delayed gastric emptying and overlapping dyspepsia has been found, but this relationship does not exist for IBS patients who do not have dyspeptic complaints. ${ }^{44}$ There is a unique relationship between the GI microbiome and gut motor function because gut microbiota modulates gut motor function, which can in turn alter the intestinal microbiota composition. ${ }^{21}$ Altered motility is most likely related to alterations in the gut microbiome, ${ }^{21}$ abnormal GI endocrine cells, ${ }^{40}$ and/or underlying GI inflammation. ${ }^{34}$

\section{$>$ Intraluminal Irritants}

Food intolerances in IBS involve pharmacologic reactions to bioactive chemicals in foods and metabolic reactions to poorly absorbed carbohydrates. ${ }^{4}$ Pharmacologic reactions to chemicals in foods include histamine, monosodium glutamate, serotonin, norepinephrine, and tyramine. These only appear to cause symptoms in a few individuals with no documented evidence that these agents play a role in IBS symptoms. ${ }^{4}$ Metabolic reactions to unabsorbed carbohydrates can occur in the colon or distal small intestine and may exacerbate IBS symptoms including diarrhea, increased flatulence, and abdominal pain. ${ }^{4}$ These poorly absorbed carbohydrates include lactose, fructose, fructans, and sugar alcohols. $^{4}$ An altered GI microbiota could contribute to symptoms of intolerance due to its role in the metabolism of nutrients, and this is specifically true for undigested 
carbohydrates. ${ }^{45}$ Dietary components other than poorly absorbed carbohydrates that are irritants for IBS patients include gluten, fat, alcohol, and coffee. ${ }^{4}$ Although it has been stated that an increased consumption of fat exacerbates IBS symptoms, there is no clear evidence. ${ }^{4}$ While some studies have evaluated the effects of coffee and alcohol in IBS, there are not reported RCTs on the effects of reduced coffee or alcohol intake for IBS symptoms. ${ }^{4}$

\section{Altered Bile Acid Synthesis}

Excess intracolonic bile acid, resulting from alterations in the enterohepatic circulation of bile acids, has been found to be an additional intraluminal irritant for IBS patients. ${ }^{46}$ Alterations occur due to low bile acid retention values, which is caused by bile acid malabsorption and/or overproduction. ${ }^{34}$ The GI microbiota is involved in the metabolic pathway of bile acids, which further supports the relationship between the GI microbiome and IBS. ${ }^{34}$

There are three types of bile acid malabsorption, with type 2 being idiopathic bile acid malabsorption (I-BAM). This type is thought to result from a genetic defect in the apical sodium bile acid transporter protein that is responsible for most of the bile acid reabsorption. ${ }^{47}$ The systematic review by Wedlake et al. ${ }^{47}$ concluded that adult onset of IBAM is actually not a rare condition and may be an important contribution to symptoms in IBS-D, with around 30\% of IBS-D patients having bile malabsorption.

\section{Inflammation}

The activation of the intestinal immune system in IBS patients has been explored, ${ }^{26,48}$ and in IBS patients, there appears to be increased innate immune activity. ${ }^{48}$ Specifically, there are increased concentrations of intraepithelial lymphocytes, mast cells, 
and 5-hydroxytryptophan-secreting enterochromaffin cells in the mucosa of IBS patients. $^{21,26}$

In humans, mast cells are important in some forms of allergy. ${ }^{49}$ As previously stated, studies have found that there is a higher concentration of mucosal mast cells in IBS patients versus healthy controls. ${ }^{21,26,35}$ Because mast cells function as both an effector and sensor, ${ }^{35}$ they appear to be essential in the activation of the innate immune system in IBS and pathology of IBS. ${ }^{48}$ Mast cells in the GI mucosa are important for wound healing and fighting pathogens. ${ }^{35,48}$ They also play a role in hypersensitivity reactions, permeability, secretion, and peristalsis. ${ }^{35,48}$ Mast cells are located closely to enteric nerves; therefore, the mast cell nerve axis can be easily activated. This is supported from the findings by Barbara et al., ${ }^{50}$ where mast cells were frequently found near intestinal nerves in both healthy subjects and IBS patients. Mast cells are closely related anatomically and functionally to GI components, and are activated by psychological stress and the ingestion of food. ${ }^{35}$ It has been found that the intestinal mucosa of both IBS and PI-IBS patients has an increased number of mast cells, and an increased number of mast cells found near intestinal nerves could contribute to increased visceral hypersensitivity and pain perception in these patients. ${ }^{51,52}$ There is no direct link between mast cells and IBS pathology, but there could be a relationship due to the increased concentration of mast cells, location of mast cells, and response of mast cells to stress and dietary intake.

It could be possible that upregulation of the GI immune system is somewhat driven by the GI microbiota. ${ }^{48}$ Gut microbiota influences mucosal inflammation in inflammatory bowel disease (IBD), Crohn's disease, and ulcerative colitis (UC). ${ }^{21,26}$ It 
has been questioned that IBS may be considered as part of the spectrum of colonic inflammation with these other disorders. ${ }^{26}$ The possibility of a genetic component as a role of IBS development has been proposed after several studies found cytokine gene polymorphisms to be common in IBS patients. ${ }^{48}$ Gonsalkorale et al. ${ }^{53}$ found that IBS patients had significantly reduced frequencies of the high producer genotype interleukin 10 (IL-10) when compared to healthy controls, which supports the hypothesis of an inflammatory component behind IBS and puts into question if there is also a genetic predisposition.

\section{$>$ Gut Microbiome}

There is evidence showing that the gut microbiome plays a role in IBS and may differ from those individuals without IBS. It has been demonstrated that the diversity of microbial population is reduced and the proportions of bacterial groups are altered in IBS patients. ${ }^{21}$ Certainly, the composition of the intestinal microbiota can vary depending on the individual, environmental factors, lifestyle, diet, drugs, stress, and invasive medical procedures. ${ }^{21}$ Furthermore, the physiological and immunological roles of the gut cannot function without the GI microbiota. ${ }^{26}$ The GI microbiota is divided into the luminal bacteria, which is dispersed in liquid feces or found in food particles, and the mucosaassociated bacteria, which is bound to a mucosa layer in the GI tract. ${ }^{26}$ The luminal microbiota potentially play a key role in the symptoms of bloating and flatulence through carbohydrate fermentation and gas production. ${ }^{26}$ The mucosa-associated microbiota can influence the host through immune-microbial interactions. ${ }^{26}$

Fecal microbiota has been found to be significantly altered in individuals with IBS when compared to healthy controls. ${ }^{54}$ Moreover, it appears that mucosal bacteria is 
more abundant in IBS patients. ${ }^{21}$ There is a theme of a relative reduction in lactobacilli and bifiobacteria in the fecal samples of IBS patients and a higher amount of enterobacteriaceae, coliforms, and bacteroides. ${ }^{26}$ These alterations in the mucosaassociated microbiota can cause an upregulation of the immune system and may contribute to the pathophysiology of IBS. ${ }^{26}$ Additionally, this reduction in lactobaccili and Bifidobacterium is detrimental because these microbiota have anti-inflammatory effects.

\section{Small Intestinal Bowel Overgrowth}

Researchers have also proposed that small intestinal bowel overgrowth (SIBO) is related to the development of IBS. ${ }^{55-56}$ There are several different causes of SIBO in the general population, including: failure of the gastric acid barrier, small intestinal dysmotility, anatomic alterations, or impaired of systematic and local immunity. ${ }^{57}$ SIBO is currently defined by $>10^{5}$ colony forming units of bacteria per milliliter of upper gut aspirate. ${ }^{57-58}$ SIBO can be classified based on differences in bacterial flora: gram positive flora or coliform flora. ${ }^{58}$ SIBO has variable clinical manifestations. Some patients may experience diarrhea, weight loss, dehydration, and nutrient deficiencies. SIBO also has similar symptoms to IBS, specifically abdominal distension, abdominal pain, and increased flatus. ${ }^{57}$ IBS-D, female gender, older age, bloating, increased flatulence, proton pump inhibitor and narcotic intake, and low hemoglobin are associated with SIBO among IBS patients. ${ }^{58}$ SIBO is commonly tested using methane or hydrogen breath testing. ${ }^{57}$

The study by Pimentel et al. ${ }^{55}$ found the high occurrence of SIBO in IBS patients. Moreover, $78 \%$ of the IBS subjects had a positive Lactulose Hydrogen Breath Test (LHBT) suggesting SIBO. The successful treatment of SIBO resulted in significant 
improvement of diarrhea and abdominal pain and elimination of IBS in $48 \%$ of the subjects. ${ }^{55}$ These results suggest that SIBO is associated with IBS and could be a potential underlying cause for this disorder. A later study by Pimentel et al. ${ }^{59}$ found that $84 \%$ of IBS patients had abnormal LHBT results compared to only $20 \%$ of healthy controls.

Other researchers has evaluated the SIBO-IBS relationship, with mixed results. ${ }^{60}$ Ford et al. ${ }^{56}$ found in their systematic review that the prevalence of SIBO in IBS patients was highest when using LHBT to measure, but new data indicates that LHBT may not test accurately for SIBO.$^{5,26,48,60}$ Lactulose passes unabsorbed into the colon and can lead to a high false positive rate because of colonic fermentation. ${ }^{26}$ Jejunal aspirate and culture is considered the gold standard for diagnosing SIBO, and only one large study using these diagnostic criteria has been published finding low rates of SIBO in the IBS subjects. ${ }^{26}$ Furthermore, the evidence that SIBO causes IBS is not causal and cannot be the only explanation for the development of IBS.

\section{Altered Brain Gut Signaling}

The hypothesis of altered brain-gut signaling expresses that IBS patients have a perceptual hypersensitivity to gut signals. ${ }^{34}$ This hypersensitivity could arise from visceral hypersensitivity, central dysregulation, or a contribution of peripheral versus central factors. ${ }^{34}$

\section{$>$ Visceral Hypersensitivity}

Hypersensitivity is an increased sensation of stimuli. IBS has been associated with rectosigmoid hypersensitivity. ${ }^{37,61}$ Hypersensitivity can lead to luminal distension, another common occurrence in IBS. ${ }^{61-62}$ The response of the enteric nervous system to 
this luminal distension is the reason symptoms can be triggered, such as abdominal pain, bloating, flatus, and changes in bowel habits. ${ }^{62}$ Researchers have evaluated many different factors that may cause visceral hypersensitivity in IBS. Adverse reactions to one or more foods is common in IBS, and the composition of a meal could increase hypersensitivity. It has been found that high fat meals have a more intense effect on rectal sensitivity versus meals high in carbohydrate content. ${ }^{37}$ However, fermentable carbohydrates are known to cause symptoms in IBS patients due to a combination of visceral hypersensitivity and dysmotility. ${ }^{63}$

Hormonal changes may also affect visceral hypersensitivity, since women with IBS have more fluctuations in symptoms during their menstrual cycle. ${ }^{37}$ In the study conducted by Houghton et al., ${ }^{64}$ women with IBS had increased rectal sensitivity and worsening of IBS symptoms during menses, while these changes in rectal sensitivity were not seen in the healthy controls during their menses. An altered visceral perceptual response has also been seen in IBS patients during and after stress. ${ }^{37}$ Murray et al. ${ }^{65}$ discovered enhanced visceral sensitivity in IBS patients and not controls during both physical and psychological stress. There is also a possibility that psychological factors affect visceral sensitivity, however, the research behind this is unclear and contradicting. ${ }^{37}$

An alternative regulatory mechanism controlling visceral sensitivity in the IBS population has been questioned. This altered mechanism could be either central or peripheral. ${ }^{37,65}$ Researchers have proposed that the gut microbiota could play a significant role in modulating visceral pain and acting as a cause of GI sensitivity in IBS. ${ }^{21}$ Mucosal mast cells have also been associated with visceral hypersensitivity specifically. When 
mast cells are activated by food or stress, they secrete mediators such as histamine and protease that cause hypersensitivity in the nerve terminals of pain-transmitting afferent neurons. ${ }^{35}$ Barbara et al. ${ }^{50}$ found activated mast cells were correlated with severity and frequency of abdominal pain and increased release of mast cell mediators histamine and tryptase was revealed. Since these mediators are known to induce visceral hypersensitivity, their release near colonic innervation suggests that they contribute to the disturbed sensory motor function. ${ }^{50}$

\section{Disturbance in the CNS-ENS Interaction}

The model known as the brain-gut axis explains how the enteric, central, and autonomic nervous systems work together with the hypothalamic-pituitary axis in health and disease. ${ }^{26}$ Alterations along the brain-gut axis in IBS patients have been questioned, but further research is necessary. ${ }^{37}$ The common IBS symptoms of abdominal pain and discomfort likely involve changes in the communication between the gut and the brain. ${ }^{21}$ The alteration of the gut-brain axis can cause a modified perception of visceral events, including hyperalgesia or allodynia. ${ }^{21}$ GI microbiota interacts with the host in a mutualistic relationship, but the presence or absence of the gut microbiota can influence the metabolic profile in other regions of the body and could affect systems involved in the gut-brain axis. ${ }^{21}$ Many studies have evaluated the brain responses to peripheral stimuli in IBS patients, specifically evaluating different pain processing regions. Research has demonstrated increased activity in sensory areas during visceral distension

and abnormal processing responses in areas involved with endogenous pain modulation. ${ }^{37}$

A dysregulation of the parasympathetic nervous system has been questioned as a contributing factor in IBS pathogenesis as well. Gupta et al. ${ }^{66}$ found heightened 
responses to cutaneous and visceral stimuli for IBS patients versus healthy controls, suggesting central autonomic dysregulation. Additionally, van Orshoven et al ${ }^{67}$ assessed autonomic function during mealtime and found results suggesting reduced baseline activity and responsiveness of the parasympathetic nervous system. Moreover, alterations in bowel habit are likely related to the dysregulation of the autonomic system in the gut. ${ }^{21}$ The impairment of parasympathetic tone could lead to greater sympathetic responses without any clear increase in sympathetic outflow, but further studies are necessary to identify the relationship of this to IBS symptoms. ${ }^{67}$

\section{Psychological Distress}

A dysregulation between the mind and body can result in illness. Furthermore, the biopsychosocial model of IBS displays how the relationship between psychosocial and physiological factors can result in the clinical outcome of functional GI symptoms. ${ }^{23}$ The most prevalent psychiatric disorders seen in IBS patients are depression, anxiety, somatoform disorders, and phobic disorders. ${ }^{23,68}$ Psychosocial factors and stressful events are related to symptom exacerbation and poor prognosis. ${ }^{1,23,68-69}$ A higher level of psychosocial stress appears to be significant in individuals with more severe IBS. ${ }^{68}$ Psychosocial factors are more strongly related to fluctuations in symptoms patterns and healthcare seeking behaviors of IBS patients rather than development of the illness. ${ }^{37}$ The exact relationship between IBS and psychosocial factors is unclear. ${ }^{1}$

\section{Medical Management of IBS}

The management and treatment of IBS is challenging due to the unknown etiology and poorly defined pathophysiology. ${ }^{21}$ Many individuals with IBS do not seek 
medical treatment. In the study conducted by Hungin et al. ${ }^{13}$ regarding IBS prevalence in the United States, it was found that while $14.1 \%$ of subjects met the diagnostic criteria for IBS, only $3.3 \%$ of subjects had a documented diagnosis of IBS. Despite the high occurrence of IBS in the United States and worldwide, it is estimated that only $10-30 \%$ of individuals with symptoms consistent with IBS seek medical care. ${ }^{14-15}$ The high prevalence of psychosocial factors in IBS makes establishing a therapeutic doctor-patient relationship crucial for optimizing care and reducing future healthcare costs. ${ }^{68}$ It has been found that patients who undergo therapy for IBS and have a therapeutic response show increases in HRQOL. ${ }^{20}$ Treatment is determined by the type of symptoms, the severity of symptoms, and any associated psychosocial issues. ${ }^{1}$ Treatment options for IBS are focused on reducing symptoms and this commonly involves pharmacologic agents, ${ }^{19}$ but these agents offer only mild palliation in the majority of patients. ${ }^{62}$

\section{Medications to Alter the GI Microbiome}

Altering the GI microbiota is an appealing therapy option since it is a likely contributing factor in the etiology of IBS. ${ }^{26}$ This includes probiotics, prebiotics, and antibiotics. ${ }^{5}$ It is difficult to interpret the benefit of probiotics in IBS due to studies employing different strains, species, doses, and preparations in various patient populations. ${ }^{5}$ Probiotics are living organisms that can provide a variety of health benefits. ${ }^{70}$ Probiotics taken as a whole can improve global symptoms, flatulence, and bloating in IBS patients, but there is conflicting data regarding individual species, preparations, and strains. ${ }^{5}$ The mechanism behind probiotics in managing IBS symptoms is through increasing mucosal TGF- $\beta$ and IL-10, and reducing proinflammatory cytokines. ${ }^{26}$ Specific probiotics can also reduce intestinal pain or alter the integrity of the 
GI mucosa. ${ }^{26}$

Prebiotics are dietary substances that are not absorbed in the small bowel and enter to colon to provide nutrients for certain bacteria. ${ }^{70}$ There are currently no RCTs of prebiotics in IBS. ${ }^{70}$ The American College of Gastroenterology states that there is insufficient evidence to recommend prebiotics in IBS. ${ }^{5}$ Prebiotics for IBS symptoms have not been adequately tested and RCTs are necessary to test the effects of various prebiotic fibers in IBS. ${ }^{4-5}$ Synbiotics, preparations containing both a probiotic and prebiotic, ${ }^{26}$ are also not recommended for use. ${ }^{5}$ The relationship between IBS and SIBO provides a rationale to evaluate the use of antibiotics in IBS. ${ }^{5}$ However, antibiotics can also be a trigger for IBS symptoms. ${ }^{26}$ It has been found that the non-absorbable antibiotic rifaximin improves IBS symptoms and bloating in IBS-D patients. ${ }^{5}$ In these trials, tests for SIBO were not performed, therefore, it is hard to determine the exact mechanism of action behind rifaximin in IBS patients. ${ }^{5}$

\section{Medications to Relax GI Smooth Muscle}

Treatments intended to relax the GI smooth muscle in IBS patients are used, such as antispasmodics, based on the assumption that colonic smooth muscle spasm contributes to IBS symptoms. Ford et al. ${ }^{5}$ reviewed 23 RCTs and found that as a class, antispasmodic therapy has a statistically significant effect in improving IBS symptoms, but the effect of individual antispasmodics is difficult to interpret. The antispasmodics otilonium, hyoscine, cimetropium, pinverium, and dicyclomine provide symptomatic short term relief in IBS patients, although it is more common for patients to experience adverse events with these medications. ${ }^{5}$ 


\section{Serotonin Antagonists and Agonists}

Select 5-HT antagonists and agonists have been reported to relieve symptoms in IBS patients since serotonin plays a role in GI secretion, sensation, and motility. ${ }^{5,35-36}$ The serotonin subtype $3\left(5-\mathrm{HT}_{3}\right)$ receptors play a role in visceral pain. Certain $5-\mathrm{HT}_{3}$ antagonists can decrease GI pain and slow intestinal transit. ${ }^{5}$ Alosetron, a selective $5-\mathrm{HT}_{3}$ antagonist, is beneficial for women with IBS-D. ${ }^{5}$ However, there is a concern for serious adverse events, including ischemic colitis and severe constipation. Alosetron is limited to women with IBS-D under the context of a carefully monitored program. ${ }^{5}$ Ramosetron, another 5- $\mathrm{HT}_{3}$ antagonist, has also shown efficacy in treating IBS symptoms. It is only available for use by IBS-D patients in Japan, Korea, and Thailand. ${ }^{5}$ When the serotonin subtype $4\left(5-\mathrm{HT}_{4}\right)$ are stimulated, they can increase intestinal secretion, supplement the peristaltic reflex, and increase GI transit. Tegaserod is the only 5-HT 4 agonist that has been tested in large RCTs for use in IBS patients, but it was removed from the market by the Food and Drug Administration (FDA) because of potential cardiovascular adverse effects. ${ }^{5}$ Other $5-\mathrm{HT}_{4}$ agonists have not been tested for use in IBS, but have shown benefits in constipation. Mixed 5- $\mathrm{HT}_{3}$ antagonists and 5- $\mathrm{HT}_{4}$ agonists that have been tested for therapeutic use in the IBS population as well. Ford et al. ${ }^{5}$ analyzed nine doubleblind, placebo-controlled trials and found no statistically significant differences between placebo and 5- $\mathrm{HT}_{3}$ antagonists $/ 5-\mathrm{HT}_{4}$ agonists in the treatment of IBS, with significant heterogeneity between studies. ${ }^{5}$

\section{Antidepressants}

Antidepressants were first introduced as a treatment option for IBS since depression and anxiety are frequent comorbidities in IBS patients. Later studies suggested 
that antidepressants are effective in relieving visceral pain. Furthermore, evidence displays that antidepressants, including both tricyclic and selective serotonin reuptake inhibitors, are effective in providing global symptom relief and reducing pain in IBS. Side effects with these medications are common, and patient acceptability has limited their use. ${ }^{5}$

\section{Medications for IBS-D Symptoms}

The anti-diarrheal medication Loperamide has been evaluated as a treatment option for IBS-D or IBS-M patients through clinical trials. The American College of Gastroenterology states there is insufficient evidence to recommend Loperamide as a treatment option for IBS patients. While Loperamide is an effective antidiarrheal, there is no evidence supporting that Loperamide relieves IBS symptoms. ${ }^{5}$

\section{Medications for IBS-C Symptoms}

Two prosecretory agents, Linaclotide and Lubiprostone, are recommended for use by the American College of Gastroenterology as effective treatment options for patients with IBS-C because they help with intestinal transit. Linaclotide, a 14-amino acid peptide, is structurally similar to hormones in the guanylin peptide family. The guanylin peptides help in the regulation of intestinal fluid and electrolyte homeostasis. ${ }^{5}$ Lubiprostone stimulates the chloride channel type 2 (CIC-2) on the apical surface of the epithelium, causing chloride and water flux into the intestinal lumen. ${ }^{5}$ Both agents were evaluated as superior to placebo in clinical trials. ${ }^{5}$ Polyethylene glycol (PEG) is an osmotic laxative that is beneficial for occasional constipation, but is not recommended for use by IBS-C patients due to a lack of evidence. ${ }^{5}$ 


\section{Complementary Health Approaches}

Approximately one in four people in the United States utilize complementary and alternative medicine (CAM), ${ }^{71}$ which were redefined as complementary health approaches by the National Center for Complimentary and Integrative Health (NCCIH). ${ }^{72}$ Patients who use complementary health approaches often have conditions not easily treated by modern medicine, and will utilize complementary health approaches in addition to conventional medicine. ${ }^{71}$ When complementary and conventional approaches are utilized together, this is known as integrative medicine. ${ }^{72}$ Because FGIDs are difficult to manage, around a third of the United States FGID patient population utilize complementary health approaches, at a median yearly cost of $\$ 200$ per patient. ${ }^{73}$ There are numerous complementary health approaches utilized by FGID patients, including aromatherapy, massage therapy, yoga, ginger root, tea, fennel seed, and evening primrose oil. ${ }^{73}$ Some common complementary health approaches for IBS patients are the use of peppermint oil and psychosocial therapies.

\section{Peppermint Oil}

Peppermint oil (PO) has been used as a treatment in IBS because it can relax smooth muscle, reduce visceral hypersensitivity, and modulate pain sensation. ${ }^{5} \mathrm{PO}$, and its major constituent, menthol, block $\mathrm{Ca}^{2+}$ channels in the GI tract and cause a relaxing effect on the GI smooth muscle. ${ }^{74}$ Enteric-coated PO may be more efficacious than smooth muscle relaxants. ${ }^{75}$ Adverse events that can be caused by PO include heartburn, and anal or perianal burning or discomfort. ${ }^{75}$ Colpermin is a safe therapeutic treatment for IBS symptoms, specifically abdominal pain. ${ }^{74,76}$ Merat et al. ${ }^{74}$ used Colpermin, an enteric-coated peppermint oil capsule, in a randomized double-blind placebo-controlled 
trial and found that subjects in the experimental group experienced a significant improvement in quality of life and no significant adverse reactions. Many subjects were free from abdominal pain, and the severity of abdominal pain was reduced significantly for those taking Colpermin. ${ }^{74}$ Since the formulation of Colpermin ensures that the active ingredient reaches the colon in an unmetabolized state, adverse effects are less likely than with traditional PO formulations. ${ }^{76}$ An earlier prospective randomized-controlled trial by Liu et al. ${ }^{76}$ also found that Colpermin controlled IBS symptoms, specifically abdominal pain, distension, stool frequency and consistency, and flatulence in about $78 \%$ of the study subjects. Neither of these studies distinguished IBS subtypes. PO, specifically in the form of Colpermin, is beneficial in controlling IBS symptoms, especially if a primary symptom is abdominal pain.

\section{Psychosocial Therapies}

Some psychological therapies have proven to improve symptoms for IBS patients. The three main approaches used for IBS patients in tertiary care centers are cognitive, behavioral, and interpersonal therapy. ${ }^{68}$ Hypnotherapy has also recently emerged as a technique ${ }^{68}$ Specific therapies shown to result in symptom improvement include cognitive behavioral therapy (CBT), hypnotherapy, multi-component psychological therapy in person and via telephone, and dynamic psychotherapy. ${ }^{5} \mathrm{CBT}$ is typically beneficial in treating anxiety and depression; therefore, if these are comorbidities that an IBS patient experiences, it may be a useful approach. By altering dysfunctional perceptions, CBT can help relieve IBS symptoms. ${ }^{68}$ There is a lack of available therapists experienced in the management of IBS; therefore, this limits the use of psychological therapies. ${ }^{5}$ 


\section{Nutritional Management of IBS}

Many individuals with IBS consider their symptoms to be related to dietary intake. In the studies by Hayes et al., ${ }^{2}$ Monsbakken, Vandvick, and Farup, ${ }^{77}$ and Simren et al., ${ }^{3}$ results showed that $90 \%, 70 \%$, and $63 \%$ of participants believed that their symptom manifestation was related to the ingestion of food. Since many patients believe their symptoms are related to the diet, this raises concern for inadequate nutritional intake or nutrient deficiencies in this population. Often, IBS patients will come up with their own theories and remedies to explain their symptom responses related to the ingestion of foods based on their own experiences. ${ }^{5}$ For example, IBS patients have been found to limit fruits and vegetables in their diet when compared to healthy controls in order to reduce symptoms. ${ }^{2}$ Hayes et al. ${ }^{2}$ conducted a dietary survey in the IBS population and found that while many patients considered their symptoms to be dietary-related, only few sought professional healthcare advice and $92 \%$ made dietary changes on their own. This involved limiting or eliminating food groups, and only $12 \%$ of patients consulted a dietitian to supervise these dietary changes. Monsbakken et al. ${ }^{77}$ found through their cross-sectional study that $62 \%$ of the IBS participants limited or excluded food from their diet, with $12 \%$ of these participants presenting an inadequate dietary intake.

Unsupervised dietary changes can lead to nutritional deficiencies and inadequate symptom relief.

\section{Dietary Patterns}

A significant association between binge eating, obesity, and symptoms of IBS has been found, ${ }^{78}$ but there is no population-based data currently available. ${ }^{79}$ A recent casecontrol study by Tigchelaar et al. ${ }^{80}$ discovered that IBS patients had a lower diet quality 
compared to healthy controls. Specifically, patients with IBS had a significantly lower energy intake than healthy controls. Intakes of fiber, fructose, and alcohol were also lower for patients with IBS. However, the intake of fat and added sugars were higher in patients with IBS compared to healthy controls. ${ }^{80}$ An excess intake of fat for IBS patients was seen in the study by Prescha et al. ${ }^{81}$ as well. Psychological distress, including anxiety, depression, and stress, are associated with IBS symptoms and could also play a factor in the eating habits of an individual. ${ }^{34}$

\section{Food Allergy}

No evidence clearly supports IBS patients suffer from food intolerance or a food allergy. ${ }^{4,40}$ Rapid onset reactions to food facilitated by immunoglobulin E (IgE) are not believed to be the cause of IBS symptoms. ${ }^{4}$ In addition, the role that cellular mechanisms and other non-IgE-mediated allergic reactions play in causing IBS symptoms is controversial. ${ }^{4}$ The therapeutic potential of food elimination based on immunoglobulin $\mathrm{G}$ (IgG) antibodies has been assessed in IBS. Atkinson et al. ${ }^{82}$ conducted an RCT where patients received either a diet eliminating all foods in which they had raised IgG antibodies or a sham diet. There was a $10 \%$ greater reduction in symptom score for patients randomized to the diet removing foods that showed raised IgG antibodies. However, this approach needs further biomedical and chemical research. ${ }^{82}$

\section{Food Intolerance}

In Western societies, the prevalence of true food allergies is between $1-3 \%{ }^{5}$ However, surveys of GI clinic patients find that $30-50 \%$ of the patients who were surveyed believe their symptoms are related to a food intolerance or allergy. ${ }^{5}$ Food- 
related IBS symptoms appear to be a food intolerance, but only $11-27 \%$ of patients can identify the problem food when asked to identify it in a double-blinded re-challenge. ${ }^{83}$

Regardless, the thought of food as a trigger for IBS symptoms is very common for both patients and healthcare providers. Traditional dietary recommendations have involved eliminating the trouble food, and specific elimination diets to alleviate IBS symptoms have included lactose-free diets and gluten-free diets. ${ }^{5}$ Carbohydrate malabsorption has been a candidate for a factor in functional GI symptoms for years, with lactose and fructose being specifically evaluated due to their osmotic activity and rapid fermentation. ${ }^{84}$ However, the malabsorption of osmotically active and rapidly fermented carbohydrates is not limited to these two groups ${ }^{84}$ There is a family of poorly absorbed short-chain carbohydrates that is now commonly termed FODMAPs, which stands for fermentable oligosaccharides, disaccharides, monosaccharides, and polyols. Further evaluation of these dietary components is still necessary, but the low-FODMAP diet has recently been a focus of dietary research for IBS symptoms and is discussed in detail later in this chapter. ${ }^{5}$

$>$ Gluten

The method to identifying IBS patients with non-Celiac gluten intolerance is not well defined. Currently, once Celiac Disease is ruled out, patients trial a gluten-free diet and then go through a rechallenge. ${ }^{85}$ Becoming gluten-free shows promise for relief of IBS symptoms, but the exact role behind this dietary change in the management of IBS still needs to be defined. ${ }^{5}$ Biesiekierski et al ${ }^{85}$ found that gluten is a trigger of gut symptoms and fatigue through a double-blind, placebo-controlled re-challenge study with IBS patients who do not have Celiac Disease. Moreover, it is vital to better understand 
the mechanism behind gluten intolerance in IBS and what factor of gluten is affecting patients. $^{85}$

Genetic predisposition is common in Celiac Disease patients, with most having positive HLA-DQ2 or HLA-DQ8 expression. ${ }^{86}$ Celiac disease associated serum IgG antibodies and HLA-DQ2 expression have been reported as predictors of clinical response to a gluten-free diet in IBS-D patients. In the study by Wahnschaffe et al., ${ }^{86}$ it was found that $37 \%$ of non-Celiac IBS-D patients have Celiac Disease associated serum IgG antibodies.

Gluten can affect mucosal barrier function in IBS-D patients, specifically those who are HLA-DQ2 or HLA-DQ8 positive. Vazquez-Roque et al. ${ }^{87}$ found that the study group receiving a gluten controlled diet had increased short bowel permeability, and this has been linked to GI inflammation and increased sensitivity. The presence of the HLADQ2 or HLA-DQ8 genotype in IBS-D patients may be a susceptibility factor for the effects of gluten on barrier function, and this also indicates that an adaptive immune response may explain this association. ${ }^{87}$ However, IBS patients who have never tried a gluten-free diet may benefit regardless of their HLA-DQ status. Aziz et al. ${ }^{88}$ found that $71 \%$ of IBS-D patients showed a clinical response to a 6 -week gluten free diet, and this reduction in symptoms was similar in both the HLA-DQ2 or HLA-DQ8 positive and HLA-DQ2 or HLA-DQ8 negative groups. While HLA-DQ status may affect the magnitude of symptom improvement, the exact mechanism behind its contribution to symptom improvement on the gluten free diet remains unclear and may actually be another component in these foods, ${ }^{88}$ such as the elimination of fructans. 


\section{Fiber}

The status of fiber in IBS nutritional therapy is not straight forward. Fiber therapy in IBS is recommended to relieve symptoms of constipation, but fiber therapy does not relieve abdominal pain. ${ }^{89}$ Insoluble fiber is known to exacerbate symptoms. ${ }^{4-5,89}$ Furthermore, the mucosal stimulating effect of insoluble particles may make diarrhea worse. ${ }^{90}$ However, soluble fibers, such as psyllium, could provide relief for IBS symptoms. ${ }^{4-5,89}$ The RCT conducted by Bijkerk et al. ${ }^{91}$ supports these recommendations, where patients receiving soluble fiber in the form of psyllium displayed symptom relief and a reduction in symptom severity in comparison to placebo, but patients receiving insoluble fiber in the form of bran experienced no symptom relief or worsening of symptoms. Furthermore, the American College of Gastroenterology Task Force on IBS concluded that psyllium was effective for IBS symptom relief. ${ }^{90}$ Psyllium provides a dichotomous effect as a stool normalizer. It can soften hard stool during constipation and improve liquid stool during diarrhea. ${ }^{90}$

\section{$>$ Carbohydrates}

The carbohydrates lactose, fructose, fructans, fructo-oligosaccharides, galactans, galacto-oligosaccharides, and polyols are short chain carbohydrates that are poorly absorbed by the small intestine for both IBS patients and healthy individuals. These carbohydrates will enter the distal small bowel and colon, increase the osmotic load, and provide a substrate for fermentation by naturally occurring bacteria. These effects can result in the production of gas, a change in bacterial populations, and a change in motility. $^{4}$ 


\section{Lactose}

Lactose is a disaccharide found in dairy products. ${ }^{4}$ The malabsorption of lactose has been evaluated in the IBS population and is not well defined. Individuals with and without IBS may report symptoms following the ingestion of lactose-containing foods. These symptoms include bloating, flatulence, abdominal distension, nausea, and loose stools. ${ }^{4}$ Lactose malabsorption or intolerance occurs when there is an inability to digest the lactose due to a deficiency in the enzyme lactase. ${ }^{92}$ The risk of lactose intolerance appears to be related to the dose of lactose consumed and intestinal gas production, and was found to be increased in IBS-D patients. ${ }^{93}$ However, providing a low-lactose diet to IBS patients has been found to have little advantage for IBS patients with lactose malabsorption. ${ }^{94}$ Study results are contradicting, and many studies have used lactose breath tests to evaluate lactose intolerance. Lactose breath testing may not indicate malabsorption, but rather an abnormal lactulose breath test, suggesting SIBO. ${ }^{95}$ Furthermore, while lactose intolerance can cause symptoms that are similar to IBS, there is no strong evidence to support a causal relationship between the two illnesses. ${ }^{4,96}$

\section{Fructose, Fructans, Fructo-oligosaccharides, Galactans and Galacto-}

\section{oligosaccharides}

Fructose is naturally present in fruits, fruit juices, honey, and table sugar. ${ }^{4}$ It is a monosaccharide is found in three main forms: as free fructose, as a constituent of the disaccharide sucrose, or as fructans. ${ }^{97}$ Data from the Third National Health and Nutrition Examination Survey (NHANES) discovered that over 10\% of Americans' daily calories are from fructose. ${ }^{98}$ Fructose can cause GI distress in all individuals regardless of an IBS diagnosis, and the amount of fructose commonly consumed in the United States could 
result in GI distress in healthy individuals. ${ }^{99}$ Fructose is incompletely absorbed in some individuals. ${ }^{9}$ When fructose is not completely absorbed in the small intestine, it will move to the colonic lumen resulting in a hyperosmolar effect. The fructose will also be fermented in the colonic lumen by luminal bacteria and cause gas production. When the osmotic effect and fermentation occur, the resulting luminal distension may cause the common IBS symptoms of bloating, abdominal pain, and changes in GI motility. ${ }^{97}$ Fructose malabsorption can be evaluated with a hydrogen breath test (HBT), where the $\mathrm{H}_{2}$ concentration is measured after ingesting fructose. ${ }^{100}$ Removal of fructose from the diet can alleviate IBS symptoms in patients who experience fructose malabsorption. ${ }^{97}$ Fructans are a polymer of fructose typically in oligosaccharide form. ${ }^{62}$ In the Western diet, wheat based food items and onions account for a majority of the fructan intake. Fructans, such as inulin, are poorly digested, making them a dietary fiber. ${ }^{4}$ Inulin has been found to distend the abdomen and increase colonic gas production. ${ }^{101}$ Fructooligosaccharides are shorter polymers of fructose with a chain less than ten fructose units. ${ }^{4}$ Fructans and fructo-oligosaccharides will resist digestion in the stomach and small intestine, causing them to travel to the large intestine where they will be available for fermentation. ${ }^{102}$ Removing fructose and its family can be successful in controlling IBS symptoms. Shepherd and Gibson ${ }^{97}$ trialed a low-fructose, low-fructan diet in 62 IBS patients and found that $85 \%$ of the patients who adhered to the diet had a significant positive symptom response. The short-chain carbohydrates galactans and galactooligosaccharides are also unable to be digested or absorbed in the small intestine because there is no enzyme in the human body that can break them down. ${ }^{9}$ Therefore, galactans 
and galacto-oligosaccharides are rapidly fermented as well, similar to the fructans and fructo-oligosaccharides. ${ }^{103}$

\section{Sugar Alcohols}

Polyols, a form of sugar alcohols, are formed through the catalytic hydrogenation of carbohydrates. ${ }^{104}$ They can occur naturally in fruits and vegetables, and are also used in sugar-free sweetened products because they yield only 0.2 to $3 \mathrm{kcal} / \mathrm{g}$, making them ideal for products aiming to decrease energy content. ${ }^{4}$ Polyols include sorbitol, mannitol, maltitol, xylitol, erythritol, polydextrose, and isomalt. ${ }^{4,103}$ Polyols are submissively absorbed in the small intestine depending on molecular size, transit time, intestinal pore size, and presence of GI disease. ${ }^{9}$ While polyol ingestion may lead to dysmotility in IBS patients, they can also cause dose-dependent symptoms of flatulence, abdominal pain, and laxative effects even in healthy individuals, as well as IBS patients. ${ }^{104}$ Only about one-third of polyols consumed of is actually absorbed. ${ }^{103}$ Polyols have been found to induce IBS symptoms independently of their absorptive pattern, therefore the restriction of polyols for IBS patients could be advantageous for reducing symptoms. ${ }^{105}$

\section{Effect of FODMAPs in the GI Tract and the Low-FODMAP Diet}

A dietary restriction of fermentable carbohydrates has shown to be effective in managing symptoms of IBS through a number of both controlled and uncontrolled clinical trials using either nutritional counseling or feeding protocols. ${ }^{6}$ This diet is referred to as the low fermentable oligosaccharides, disaccharides, monosaccharides, and polyols (FODMAP) diet. FODMAPs are a family of short chain carbohydrates, that are

poorly absorbed in the small intestine and arrive in the colon undigested. ${ }^{63}$ These families 
include foods that have excess lactose, fructose, fructans and/or galactans, and polyols. ${ }^{103,106}$ The maldigestion and malabsorption of FODMAPs occurs as a result of either reduced concentration of hydrolase enzymes for adequate digestion or incomplete absorption of some monosaccharides and disaccharides. ${ }^{6}$ While FODMAPs go through the same digestive process in both healthy individuals and the IBS population, only the IBS population appears to experience symptoms in part to the proposed visceral hypersensitivity and altered motility. ${ }^{63}$ Furthermore, FODMAPs induce symptoms more readily in IBS patients versus those without a FGID. ${ }^{107}$

One established mechanism by which FODMAPs are proposed to induce symptoms is the augmentation of small intestinal water content. ${ }^{108}$ This has been demonstrated through studies with MRI content and ileostomy recovery. ${ }^{109-110}$ FODMAPs have been found to increase the delivery of water and fermentable contents to the proximal colon. ${ }^{109}$ Specifically, fructose appears to cause an increase in small bowel water content (SBWC). ${ }^{10-111}$

Another established mechanism by which FODMAPs are proposed to induce symptoms is through fermentation of the non-digested and non-absorbed short chain carbohydrates, causing colonic gas production. ${ }^{108}$ This likely leads to luminal distension, which provokes symptoms, especially in IBS patients with visceral hypersensitivity. ${ }^{108}$ Ong et al. ${ }^{107}$ found that FODMAPs increase $\mathrm{H}_{2}$ production in the intestine for both patients with IBS and healthy individuals. However, FODMAPs caused symptoms for patients with IBS, while healthy controls only experience increased flatus with minimal physical complaints. ${ }^{107}$ Specifically, fructose and inulin appear to cause increased $\mathrm{H}_{2}$ production and colonic gas. ${ }^{110-111}$ Colonic hypersensitivity, rather than increased gas 
production and luminal distension, may drive the FODMAP-related symptoms in some IBS patients. ${ }^{112}$ The study by Major et al. ${ }^{111}$ found that patients with IBS had symptomatic responses to inulin related to levels of intraluminal gas, but peak gas levels did not affect symptom response. This finding indicates that colonic hypersensitivity produces symptoms in patients with IBS rather than excessive gas production. ${ }^{11}$

Removing poorly absorbed dietary components that cause an osmotic effect on the bowel and cause excess gas production due to fermentation by intestinal bacteria could be a beneficial dietary solution. ${ }^{63,109}$ Recent studies have purported success of the low-FODMAP diet in relieving IBS symptoms, and demonstrated that a low-FODMAP diet can effectively reduce symptoms in about $75 \%$ of patients. ${ }^{113-114}$ Since not all IBS patients respond to FODMAP challenge, it is hypothesized that these symptoms occur only in individuals with visceral hypersensitivity. ${ }^{9}$ Although the diet does show clinical effectiveness in alleviating IBS symptoms, further research is necessary to evaluate potential consequences that this dietary approach could have on long-term health. There is also an inconsistency in study protocols, including design, methodology, instrumentation, and length of the elimination phase.

\section{Symptom Relief on the Low-FODMAP Diet}

Restricting FODMAP intake globally, rather than individually, has shown to optimize symptom control in IBS patients. ${ }^{62,115}$ When implementing the low-FODMAP diet, there should be an initial elimination phase where all high-FODMAP dietary choices are completely removed from the diet. ${ }^{6,116}$ The implementation of such a restrictive diet should be monitored by a registered dietitian nutritionist (RDN). ${ }^{117}$ There has been no specific recommendation developed for how long patients should follow the elimination 
phase, ${ }^{116}$ however, most clinicians recommend a two to eight-week period. ${ }^{10,63,113}$ The elimination phase is to be followed by a reintroduction phase where patients will reintroduce high-FODMAP dietary choices from a single group at a time and assess symptoms to rule out poorly tolerated foods. ${ }^{6,116}$ This phase is very individualized and will require guidance from a RDN as well. ${ }^{116}$ There is a lack of RCTs that evaluate the reintroduction phase of the low-FODMAP diet. ${ }^{63}$ The final phase is the FODMAP personalization phase, which aims to improve diet variety and nutritional adequacy over time while maintaining symptom control. ${ }^{9}$

Over the past several years, the elimination phase of the low-FODMAP diet has shown to be effective in treating IBS symptoms through multiple clinical trials. A recently published meta-analysis by Schumann et al. ${ }^{118}$ evaluated nine RCTs and discovered significant effects of the low-FODMAP diet in improving symptom severity, abdominal pain, and HRQOL. Marsh, Eslick, and Eslick ${ }^{119}$ conducted a systematic review and meta-analysis evaluating six RCTs and sixteen non-randomized trials that evaluated for IBS symptom improvement with the low-FODMAP diet. There were significant decreases in IBS-Symptom Severity Score (IBS-SSS) for patients following the low-FODMAP diet found in both the RCTs and non-randomized trials. ${ }^{119}$ Furthermore, following a low-FODMAP diet also resulted in a reduction of abdominal pain, bloating, and overall symptoms for the RCTs and non-randomized interventions. ${ }^{119}$ Regarding specific IBS symptom improvements, Altobelli et al. ${ }^{120}$ found through their meta-analysis that the low-FODMAP diet appears to be most effective in reducing abdominal pain and bloating, while stool consistency and frequency is not affected as much. The meta-analysis by Varjú et al. ${ }^{121}$ displayed that while both standard dietary 
advice and the low-FODMAP diet prove to be effective in lowering IBS-SSS for patients, post-diet IBS-SSS scores were significantly lower for those patients following a lowFODMAP diet. Krogsgaard et al. ${ }^{63}$ conducted a systematic review evaluating the quality of current RCTs published regarding the low-FODMAP diet's effect on IBS symptom relief. The trials included in this systematic review had a low-FODMAP diet elimination period lasting from two days to six weeks. ${ }^{63}$ While five of the nine trials reported a positive effect on symptom relief when comparing the low FODMAP diet group to the control group, ${ }^{107,122-123}$ it was found that the trials included in the review may have a high risk of bias and symptomatic effects reported could be driven by the placebo response. ${ }^{63}$ The four trials that did not report a positive effect on symptom relief reported an equivalent symptomatic effect when compared to controls. The control interventions in these specific studies include hypnotherapy, ${ }^{124}$ modified National Institute for Health and Care Excellence (mNICE) guidelines, ${ }^{125}$ traditional dietary advice, ${ }^{126}$ and the probiotic Lactobacillus rhamnosus GG. ${ }^{127}$

The gold standard methodology for analysis for the efficacy of a dietary intervention is to feed subjects all or almost all their food through an RCT with a crossover design. ${ }^{8}$ Three studies have been published following this standard..$^{107,122,128}$ The pivotal cross-over, single-blinded RCT conducted by Halmos et al. ${ }^{122}$ compared a diet low in FODMAPs with a typical Australian diet in 30 IBS patients and 8 healthy controls. Subjects received the diets in a random order for 21-days with a 21-day washout period in between the two diets. Approximately $70 \%$ of the IBS patients saw symptom relief on the low-FODMAP diet, and individual IBS symptoms all scored significantly lower on the low-FODMAP diet. ${ }^{122}$ The healthy controls saw no overall change in symptoms on 
either of the dietary interventions, and $71 \%$ of the controls were unable to correctly identify which dietary intervention was the low-FODMAP diet, ${ }^{122}$ demonstrating the success of blinding in this study. ${ }^{8}$ Two additional cross-over studies have been reported, both consisting of a two day intervention period for each dietary intervention being used. ${ }^{107,128}$ One cross-over RCT compared a low-FODMAP diet to a high-FODMAP diet in order to better understand the mechanism behind the low-FODMAP diet for IBS symptom relief. ${ }^{107}$ Dietary FODMAPs were noted to produce prolonged hydrogen production in the intestine that was greater in the IBS patients. ${ }^{107}$ Hydrogen is a product of intestinal fermentation and displays that enhanced luminal distension occurred for these patients. ${ }^{107}$ The high-FODMAP diet induced GI symptoms and systemic symptoms in the IBS group, but this reaction was not seen in healthy controls. ${ }^{107}$ Another cross-over RCT evaluated the effect of the low-FODMAP diet in children with IBS, and compared this diet to the Typical American Childhood Diet (TACD). The low-FODMAP diet decreased abdominal pain episodes in children when compared to the TACD and reduced symptoms from baseline. ${ }^{128}$

Feeding studies do not mock real-world conditions where individuals are able to make their own dietary choices. ${ }^{8}$ Six RCTs have used instruction by a dietitian when implementing the elimination phase of the low-FODMAP diet. ${ }^{123,124-127,129}$ Of these studies, two reported a positive symptom relief when compared to healthy controls ${ }^{123,129}$ and the other studies reported equivalent symptom relief when compared to an alternative intervention being used as each study's control. ${ }^{124-127}$ Moreover, there have been multiple retrospective studies that report the efficacy of the low-FODMAP diet for symptom relief 
in IBS patients. ${ }^{8}$ These studies have come from diverse areas of the world, including North America, Mexico, Australia, New Zealand, and the UK. ${ }^{8}$

One study has reported the long-term implications of following a low-FODMAP diet after reintroduction. In this study by O'Keeffe et al., ${ }^{130}$ of the 103 participants enrolled, 12\% reported satisfactory symptom relief at baseline, $61 \%$ at short-term followup (post FODMAP elimination phase), and 57\% at long-term follow up. Eighty-two percent of the participants continued the adapted low-FODMAP diet at long-term follow up. Abdominal pain, bloating, and flatulence were reported by over $60 \%$ of participants at baseline and decrease by at least a third in the long-term follow up. ${ }^{130}$

A smaller number of studies have compared the outcome of the low-FODMAP diet on different IBS subtypes and symptoms. One such study found symptom improvement in all four IBS subtypes and a greater satisfaction with stool consistency among IBS patients while on the low-FODMAP diet. ${ }^{122}$ Another study evaluated symptom response for IBS patients from the low-FODMAP diet compared to the National Institute for Health and Care Excellence (NICE) guidelines between subjects. ${ }^{10}$ There was no difference in the prevalence of constipation, diarrhea, bloating, and abdominal pain among intervention groups. ${ }^{10}$ A positive trend was found for more IBS-D patients in the low-FODMAP group to report symptom improvement in diarrhea. Moderate and substantial improvement was statistically significant when results were analyzed for the magnitude of improvement in the symptom of diarrhea. ${ }^{10}$ There was no significant difference in the proportion of patients reporting improvement in constipation in either intervention group, but when results were analyzed for the magnitude of improvement in the symptom of constipation a statistically significant higher proportion 
of patients reported moderate improvement in the low-FODMAP diet group. ${ }^{10}$ In a study comparing the low-FODMAP diet to the probiotic Lactobacillus rhamnosus GG (LGG) or a normal diet, it was found that IBS-D and IBS-M patients experienced a significant reduction in symptom severity from baseline to week six with the low-FODMAP diet and LGG. ${ }^{127}$ There was no significant reduction for IBS-C patients for any of the three treatments. ${ }^{127}$ Further research is necessary to evaluate the benefits of the low-FODMAP diet for symptom improvement among specific IBS subtypes.

There are currently no clear clinical predictors for who will respond to the lowFODMAP diet. ${ }^{8}$ IBS subtype cannot be used since symptom relief has been seen amongst all subtypes and has not been specified to be better in a certain IBS subtype; ${ }^{10,122}$ however, it appears that the low-FODMAP diet may be most effective in the IBS-D subtype. ${ }^{127-131}$ De Roest et al. ${ }^{132}$ suggested hydrogen/methane breath testing for fructose and lactose malabsorption as a predictor for a positive response to the low-FODMAP diet. ${ }^{8}$ Chumpitazi et al. ${ }^{128}$ and Valeur et al. ${ }^{133}$ suggested that the gut microbiota could be used to predict responders to the diet, however, this is a long way from practical application. ${ }^{8}$ In the future, it would be desirable to discover patients likely to respond to the low-FODMAP diet therapy since this approach is cumbersome and requires changes in dietary habits. ${ }^{8}$

\section{Effect of the Low-FODMAP Diet on HRQOL}

While the low-FODMAP diet is meant to improve patients' symptoms and should therefore be expected to improve HRQOL, the opposite may occur due to the complexity of the diet. ${ }^{6}$ One study evaluated the potential for increased burden of the low-FODMAP diet, discovering that $64 \%$ of subjects in a cohort following the diet for a median of 17 
months reported an increased cost of food ${ }^{84}$ However, both a meta-analysis and a systematic review specifically analyzing HRQOL in IBS patients displayed significant improvements in scores from RCTs and non-randomized trials. ${ }^{118-119}$

\section{Effect of the Low-FODMAP Diet on the Gut Microbiome}

Another safety concern associated with the low-FODMAP diet is its effects on the gut microbiome. While it is proposed that the gut microbiome may be associated to the pathogenesis of IBS, treatments to relieve symptoms in IBS could potentially affect the gut microbiome in an unhealthy way. ${ }^{129}$ Several researchers have examined various effects of the low-FODMAP diet on GI microbiota. The low-FODMAP diet has decreased the concentration of the luminal Bifidobacterium in the GI tract. ${ }^{123,129,134}$ This is of no surprise since it is known that fructans ${ }^{6,135}$ and galacto-oligosaccharides ${ }^{6}$ have a prebiotic effect and increase the luminal Bifidobacterium. There is a restriction of fermentable carbohydrates like these on the low-FODMAP diet. However, luminal bacteria, such as Lactobacillus and Bifidobacterium, are beneficial because they stimulate dendritic cell IL-10 and TGF- $\beta$ production and inhibit the release of proinflammatory cytokines. ${ }^{26}$ There is further research necessary to evaluate if concurrent strategies, such as prebiotics or probiotics, can ameliorate the effects of the low-FODMAP diet on GI microbiota such as Bifidobacterium..${ }^{6}$ Staudacher et al.${ }^{134}$ discovered that the effect of the low-FODMAP diet on Bifidobacterium can be modified by adjunctive probiotic therapy. In this $2 \times 2$ factorial, there was a significant reduction in abundance of Bifidobacterium from baseline to follow-up that did not occur in the sham group; however, there was a significant increase in Bifidobacterium for those following a low-FODMAP diet and 
utilizing multi-strain probiotic versus those following a low-FODMAP diet without a probiotic. $^{134}$

The study by Halmos et al. ${ }^{114}$ found that the low-FODMAP diet can reduce total GI bacterial abundance, but this reduction was not seen specifically with Bifidobacterium. Studies have also evaluated the change in diversity of GI microbiota after following a low FODMAP diet. Several studies have found that there is no change in alpha or beta diversity of the microbiome after following a low-FODMAP diet. ${ }^{128-}$ ${ }^{129,131,134}$ While McIntosh et al. ${ }^{129}$ found no change in alpha or beta diversity after three weeks following the low-FODMAP diet, when comparing fecal samples from just the final week on the assigned diets, there was an increase in Actinobacteria richness and diversity in the low-FODMAP group compared with the high-FODMAP group.

The low-FODMAP diet can provoke major changes in metabolites, specifically histamine, p-hydroxybenzoic acid, azelaic acid. Histamine is a measure of immune activation, and is known to be increased in the mucosa of many IBS patients. The study by McIntosh et al. ${ }^{129}$ found an eight-fold reduction in histamine in the low-FODMAP diet group, while there was an increase in histamine seen in the high-FODMAP diet group. Studies have also evaluated changes in fermentation byproducts, specifically short chain fatty acids (SCFA). No change in fecal SCFA have been observed with compliance to the low-FODMAP diet. ${ }^{14,123}$ Further research is necessary to continue determining the potential negative effects of the low-FODMAP diet on the GI microbiota composition and to determine the effect of the low-FODMAP diet on fermentation byproducts such as SCFAs. Determining the shortest length of time required for the elimination phase before 
symptom improvement could assist in preventing undesirable outcomes on the GI microbiome.

\section{Patient Compliance to the Low-FODMAP Diet}

The low-FODMAP diet has been described as difficult to follow, and adhering to the diet is crucial in finding symptom relief. ${ }^{84,132}$ A subgroup of subjects $(n=36)$ in the study by Staudacher et al. ${ }^{10}$ were asked about their level of compliance to the diet, with $64 \%$ reporting following the diet strictly and $30 \%$ reporting at least half of the time. The prospective observational study by de Roest et al. ${ }^{132}$ found that most patients reported ongoing compliance with the diet, and there was an significant positive correlation between compliance to the diet and improvement in bloating, abdominal pain or discomfort, flatulence, diarrhea, constipation, and energy levels. Moreover, $60 \%$ of these patients reported that the low-FODMAP diet was easy to follow. ${ }^{132}$ Table 2 provides a summary of low-FODMAP diet studies evaluating compliance.

Registered Dietitians trained to provide the low-FODMAP diet education appear to have no difficulty teaching the diet to patients based on study results, ${ }^{8}$ and it has been reported that consultation(s) with a Registered Dietitian and written information are the most important factors in maintaining compliance to the diet. ${ }^{132}$

\section{Nutritional Adequacy of the Low-FODMAP Diet}

While recent studies have indicated that the restriction of FODMAPs reduces IBS symptoms, it is important that patients engage in the reintroduction phase rather than adopting total FODMAP exclusion. Since the low-FODMAP diet is highly restrictive of fruit, vegetables, dairy, and grains, this increases concern for nutrient deficiencies. There is a need to assess the nutritional adequacy of the low-FODMAP diet to evaluate the 
safety and ethicality of the elimination phase, but few have been found in this area specifically. Several RCTs that have evaluated the nutritional adequacy of the lowFODMAP diet have found that it reduced the intake of carbohydrates. ${ }^{123,125-126,136}$ This result is expected due to the restrictive nature of the diet. The fiber intake of subjects following a low-FODMAP diet is not different when compared to controls in some RCTs, ${ }^{123,125}$ however, one RCT did find a reduction compared to controls. ${ }^{126}$ Harvie et al. ${ }^{131}$ also saw a reduction in fiber intake, but this was resolved after reintroduction of FODMAPs that contain fiber. The total protein and fat intake after following a lowFODMAP diet was also unchanged. ${ }^{123,125-126}$ This is expected since the low-FODMAP diet focuses on the reduction of carbohydrates.

Variable results have been found regarding overall energy intake. In studies comparing the low-FODMAP diet to traditional IBS dietary advice, reduced energy intake was seen in both study groups. ${ }^{125-126}$ Vincenzi et al. ${ }^{136}$ evaluated the lowFODMAP diet compared to a specific carbohydrate diet, and while reduced energy intake was seen in both groups, only the low-FODMAP diet group showed a significant reduction in energy. Harvie et al. ${ }^{131}$ found a significant reduction in energy intake for both groups during the three-month low-FODMAP elimination phase. After reintroduction of FODMAPs in group 1, there was an increase in energy intake during the six-month follow-up. ${ }^{131}$ The low-FODMAP diet has also been found to be no different in energy value compared to controls following a habitual diet. ${ }^{123}$ However, it is also unknown whether energy intake is maintained in patients who self-restrict their diet and do not seek a dietitian for guidance. ${ }^{6}$ Further research is necessary to better understand the low-FODMAP diet's effect on macronutrient and overall energy intake. 
Micronutrient deficiencies are also a concern for a restrictive diet. There is a lack of data regarding the effects of the low-FODMAP diet on specific micronutrients. Trials thus far have shown calcium intake appears to be the main micronutrient affected when following the low-FODMAP diet. $^{6} \mathrm{~A}$ lower calcium intake was distinct in subjects following the low-FODMAP diet versus a habitual diet in the RCT conducted by Staudacher et al., ${ }^{123}$ however, this effect was not seen in the study by Harvie et al. ${ }^{131}$ Dietary intake of calcium has been found to be lower than recommended intakes for many patients following a low-FODMAP diet. ${ }^{137}$ The low-FODMAP diet is restrictive in dairy products due to their lactose content, therefore it is necessary for proper inclusion of low-lactose high-calcium alternatives when following the low-FODMAP diet. ${ }^{6}$ Many dietary choices that are enriched/fortified with iron are restricted on the low-FODMAP diet. Staudacher et al. ${ }^{137}$ found a significant reduction in mean iron intake from baseline to follow up for participants following a low-FODMAP diet. However, the proportion of participants following a low-FODMAP diet that were not meeting dietary reference values for iron did not differ from baseline to follow-up. ${ }^{137}$ Vincenzi et al. ${ }^{136}$ evaluated the serum levels of vitamin D and folic acid after subjects followed a low-FODMAP diet for three months and did not see any deficiencies with respect to the specific carbohydrate diet.

Current research shows that carbohydrates, calcium, and overall energy intake may be reduced in the short-term when following the low-FODMAP diet, specifically during the elimination phase. There is need for further research on the nutritional adequacy of the elimination phase regarding both macro- and micro-nutrients. While the study by O'Keeffe et al. displayed that nutritional adequacy is not compromised when 
following an adapted long-term low-FODMAP diet, ${ }^{130}$ there still a need for further research regarding long-term dietary intake data and nutritional adequacy of the lowFODMAP diet after the reintroduction phase occurs. ${ }^{6}$

\section{Necessary Duration of the Low-FODMAP Diet}

There is unclear evidence outlining how long the low-FODMAP diet elimination phase must be followed to see symptom relief. Because of the potential negative effects of the low-FODMAP diet on the GI microbiota and overall nutritional adequacy, finding the shortest elimination phase possible before reintroduction can occur is desirable. In the clinical setting, patients typically see symptom relief during the elimination phase from two to eight weeks, ${ }^{10}$ with the elimination phase usually lasting for at least four to eightweeks. ${ }^{63,113}$ An RCT comparing the low-FODMAP diet with gut-directed hypnotherapy found that $71 \%$ of the patients in the low-FODMAP intervention group had symptom improvement at 6 weeks, and $82 \%$ of these patients maintained improvement six months later despite the fact that $>90 \%$ successfully reintroduced FODMAPs. ${ }^{124}$ One study asked subjects the time taken for symptom resolution, with a mean report of 3.5 weeks

(range $=2-8$ weeks).$^{10}$ Finding a more definite duration for the elimination phase can help improve the clinical guidelines, strengthen the research, and increase the overall understanding of the low-FODMAP diet.

\section{Limitations and Need for Further Research}

Since IBS is complex and can be treated in multiple ways, this dietary treatment approach needs methodical consideration. A definitive answer regarding the question if the low-FODMAP diet should be used as first-line therapy to alleviate IBS symptoms 
cannot yet be provided. ${ }^{8}$ Thus far, the low-FODMAP diet has excellent rates of efficacy in treating IBS symptoms. ${ }^{113}$ Nevertheless, following the low-FODMAP diet for an extended period of time is not recommended due to its restrictiveness. ${ }^{103}$ There is limited data regarding long-term results and the diet's effect on the GI microbiota. ${ }^{8}$ More distinct guidelines for the duration of the elimination phase is also necessary to help decrease the chance for potential negative outcomes due to the restrictive nature. Furthermore, it is necessary to better understand the nutritional adequacy of the low-FODMAP diet during the elimination phase since there is a lack of strong research in this area. More long-term data regarding the nutritional adequacy of the low-FODMAP diet once the reintroduction phase begins is also needed.

While it appears that this nutrition intervention is ready to be used in the clinical setting as a nutrition therapy for IBS, continued research and surveillance of outcomes is warranted. ${ }^{8}$ This study will focus on the nutritional adequacy and duration of the lowFODMAP diet's elimination phase. By developing a better understanding of the nutritional adequacy and necessary length of time for the elimination phase, healthcare providers can have an increased understanding of the low-FODMAP diet and more confidence in recommending it to patients.

\section{Conclusion}

IBS is a prevalent illness that significantly decreases affected individuals' HRQOL and offers a considerable economic impact. The medical, nutritional, and alternative management approaches of IBS are not adequately supported by evidence. Since the intake of food is related to exacerbation of symptoms, an effective dietary approach for symptom improvement is needed and the low-FODMAP diet has recently 
gained attention. As a novel dietary approach for symptom relief in IBS, the lowFODMAP diet requires further research to improve guidelines for clinical recommendations. Due to this diet's restrictive nature, prolonged elimination raises concern regarding nutritional adequacy. Determining the shortest elimination phase period necessary for optional symptom relief is vital. A better understanding of the elimination phase can help ensure the provision of the least restrictive diet possible. 

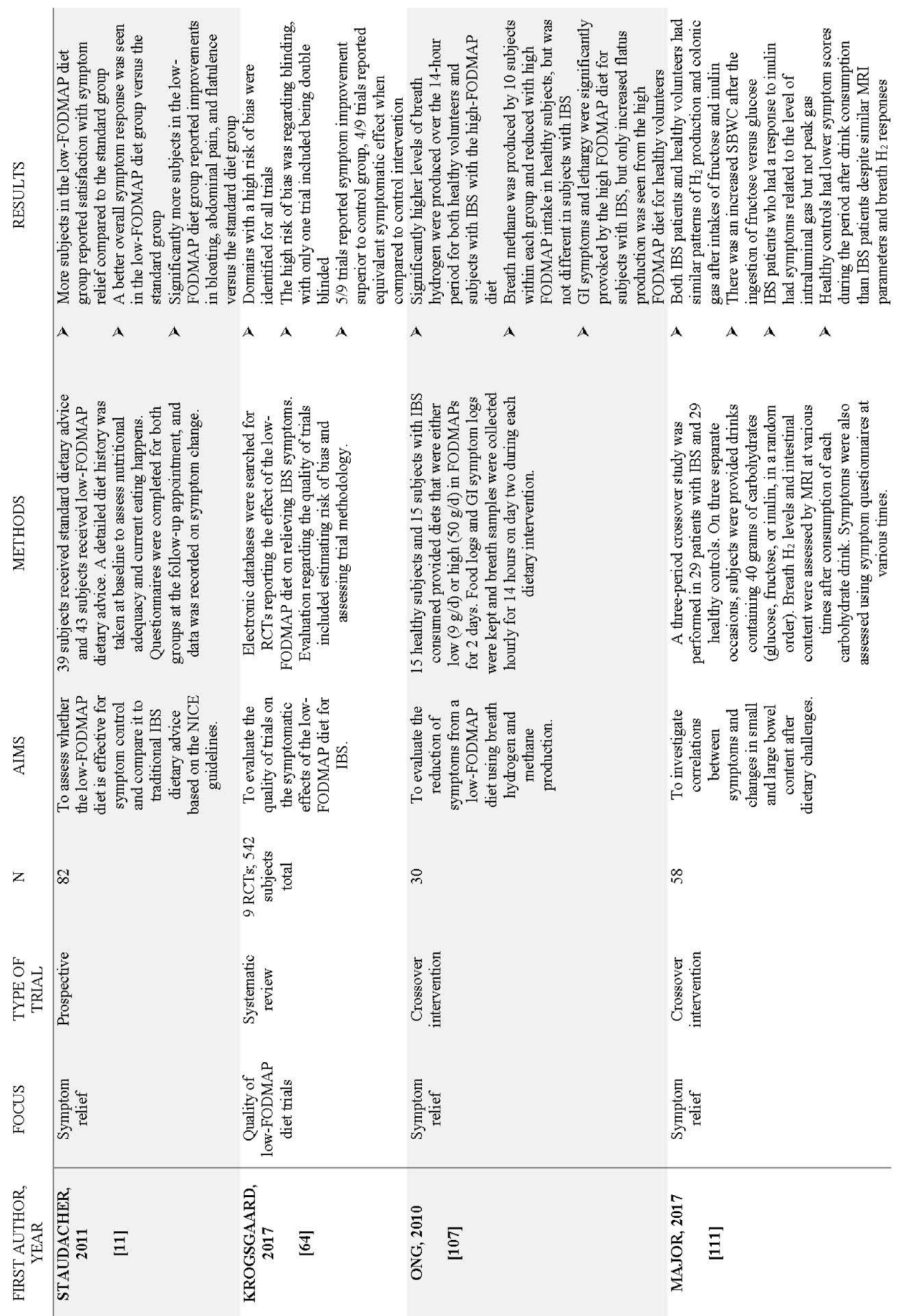

Table 1. Summary of Primary Research Studies on the Low-FODMAP Diet in Patients with IBS 

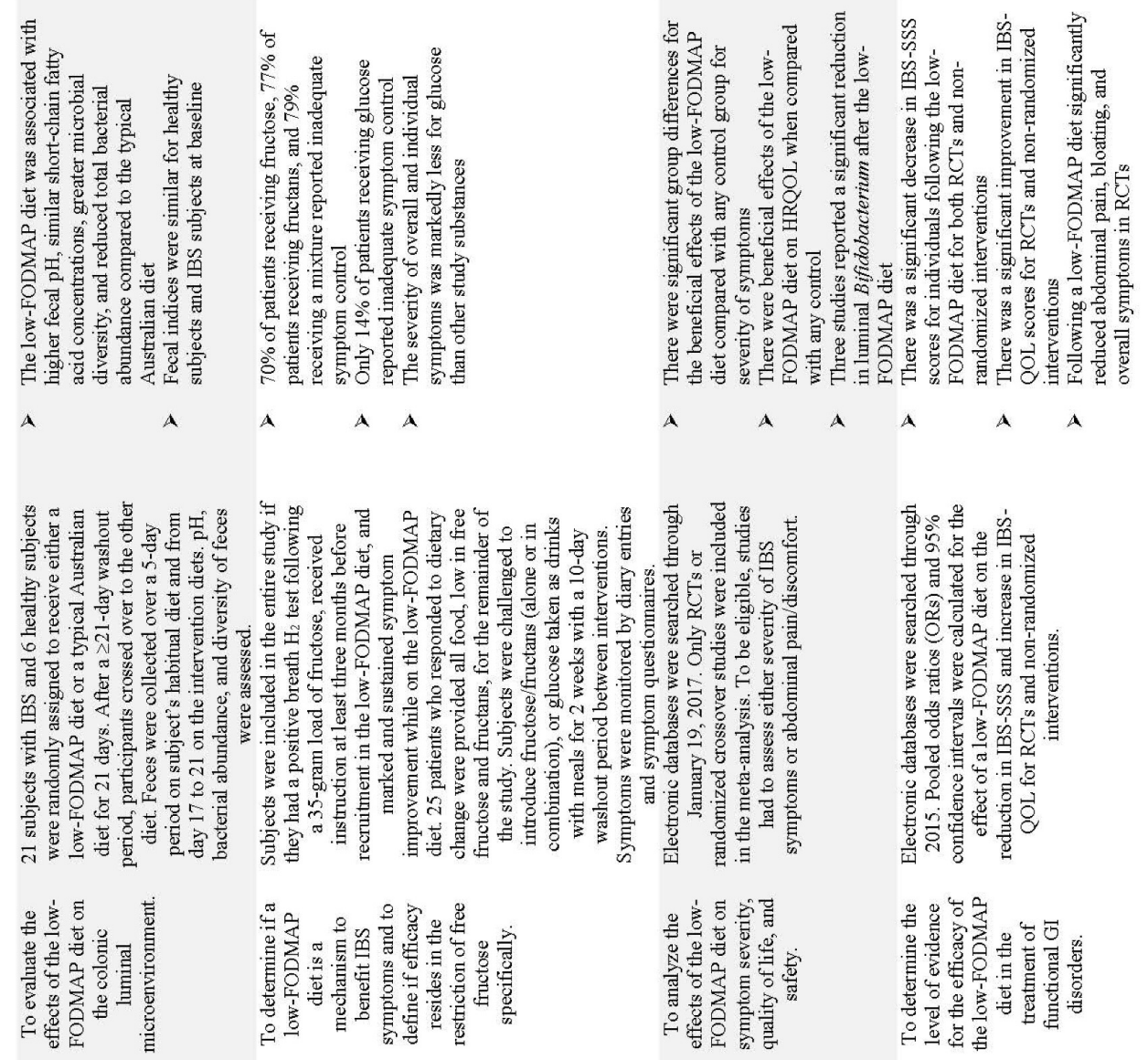

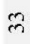

누
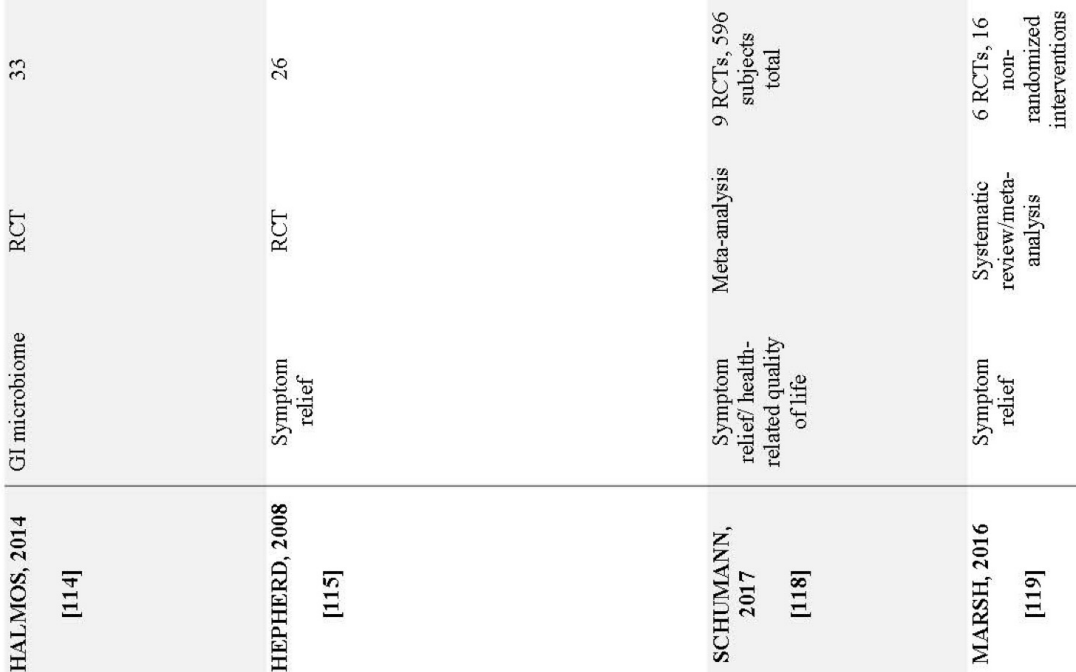

홍

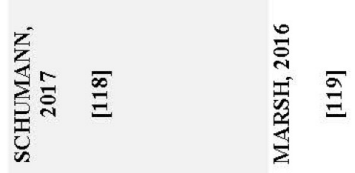



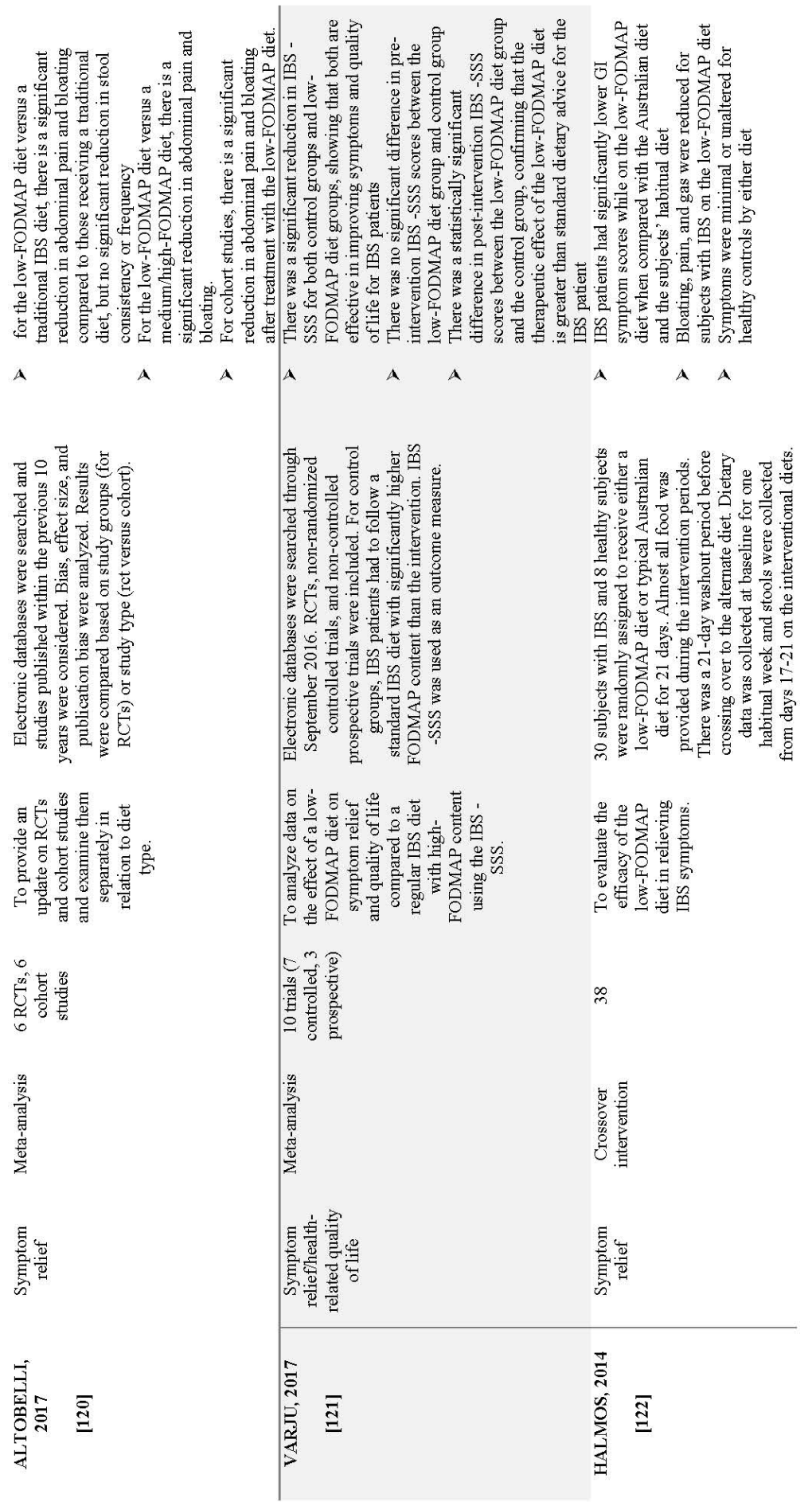

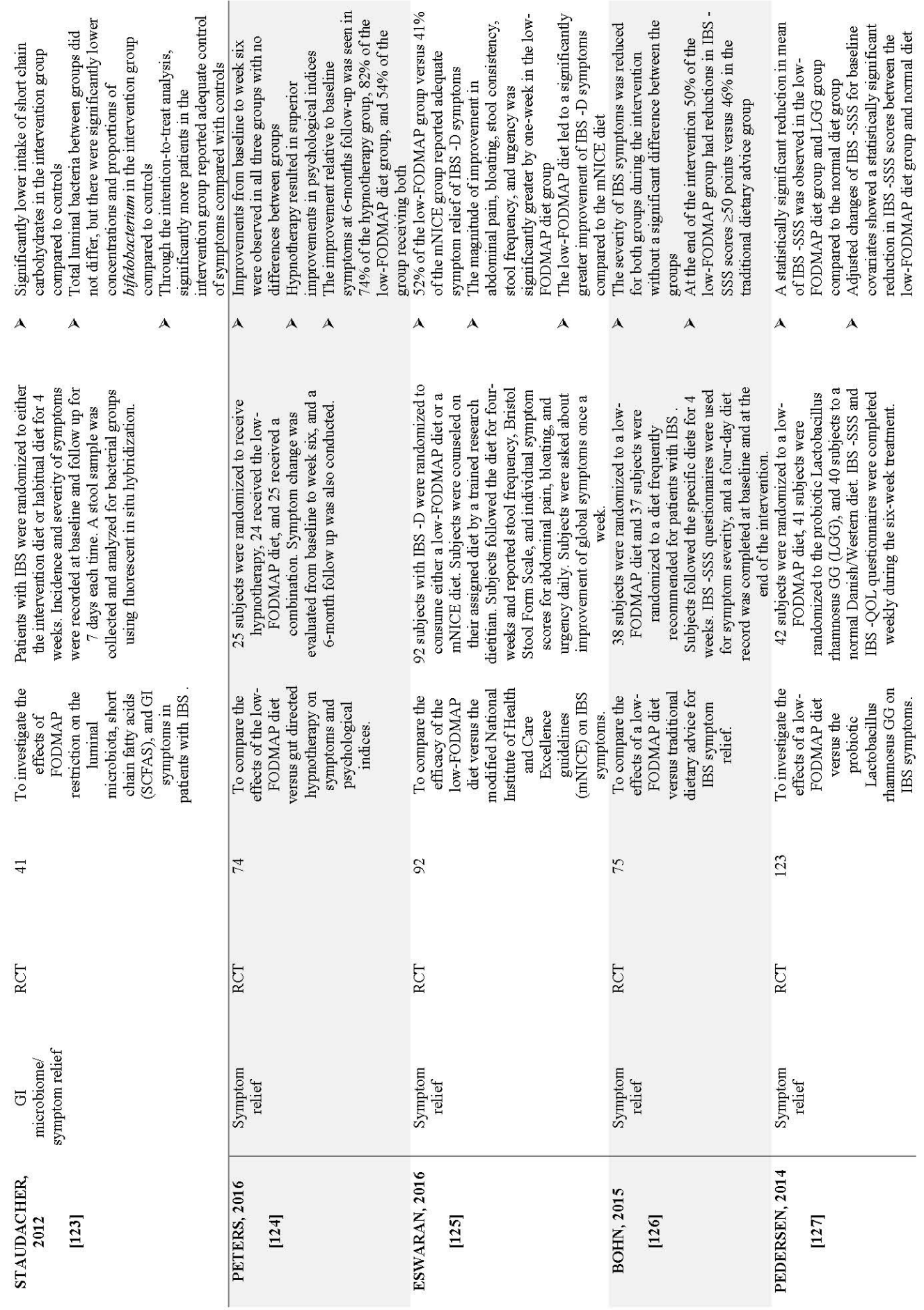

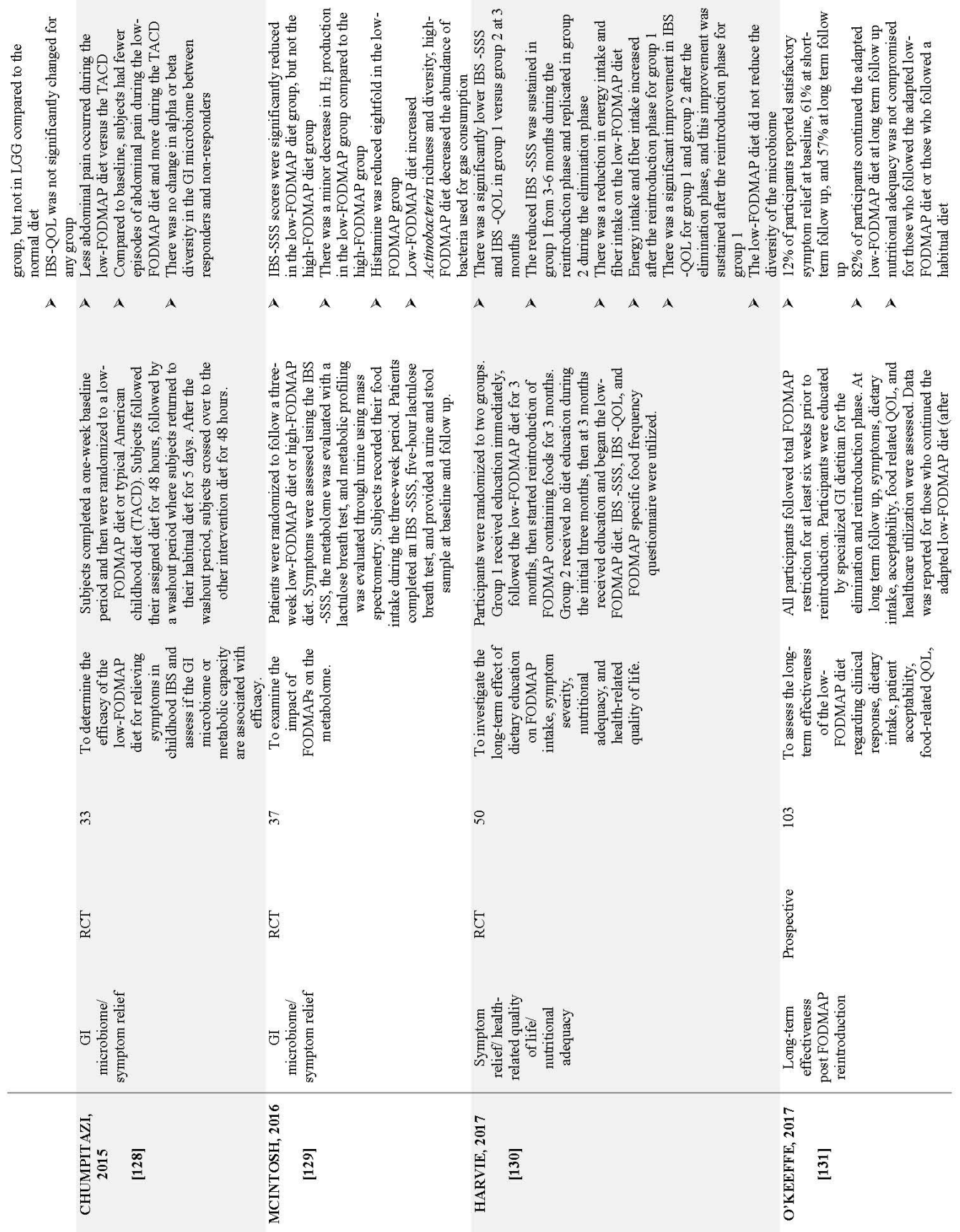

...Continued 

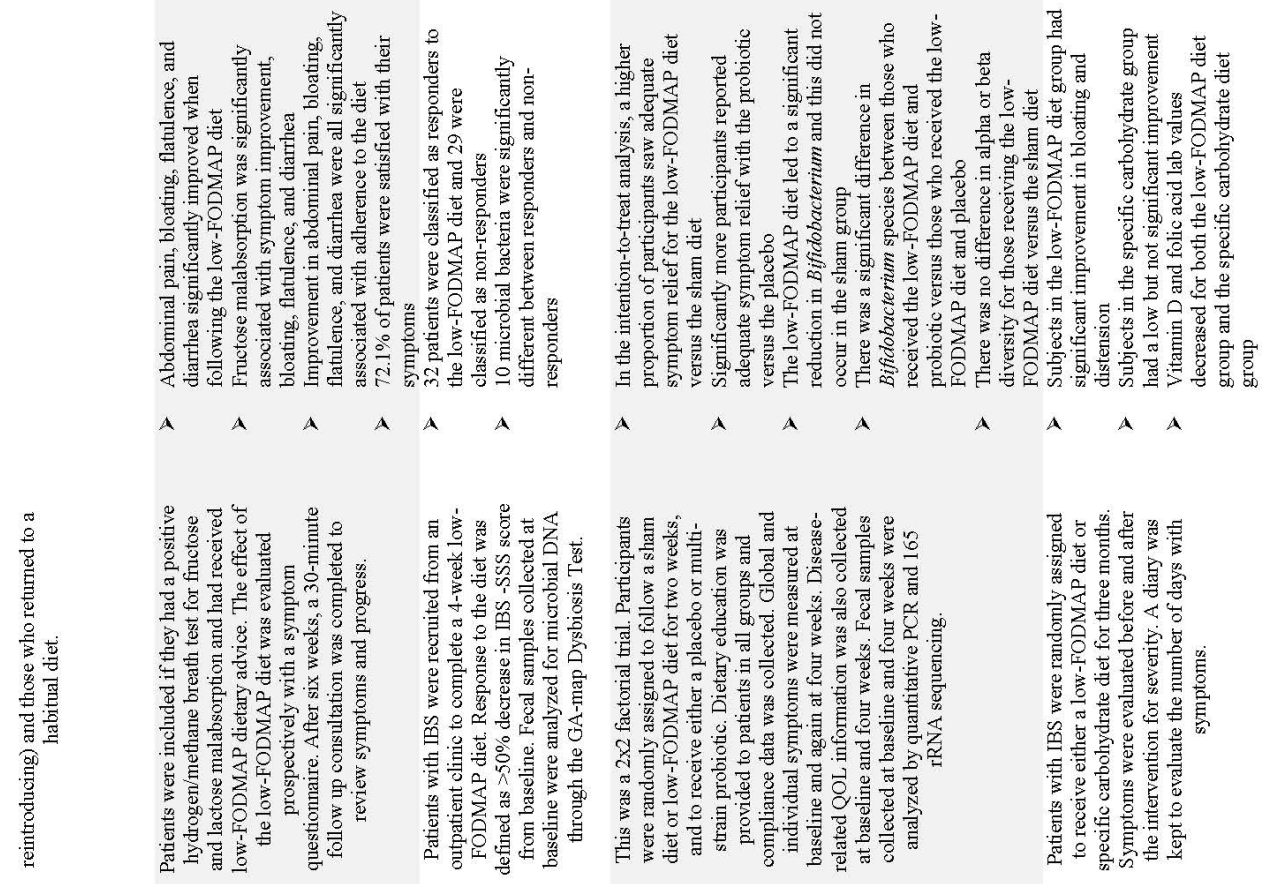

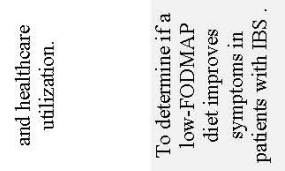
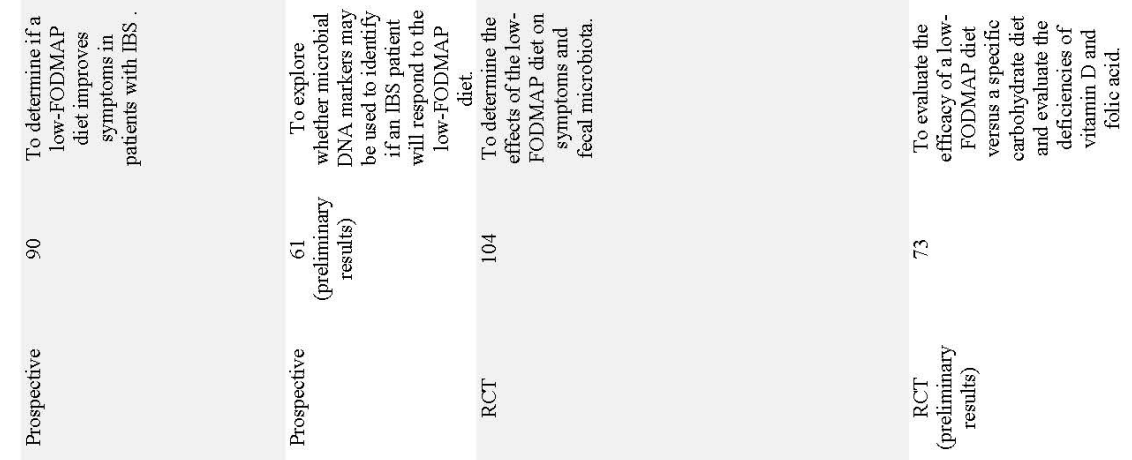

$\Xi$

2

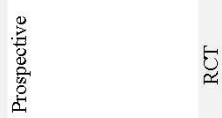

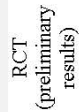
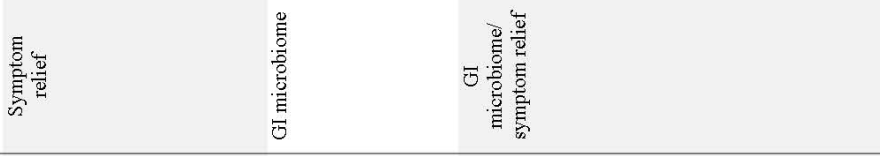

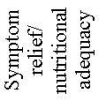

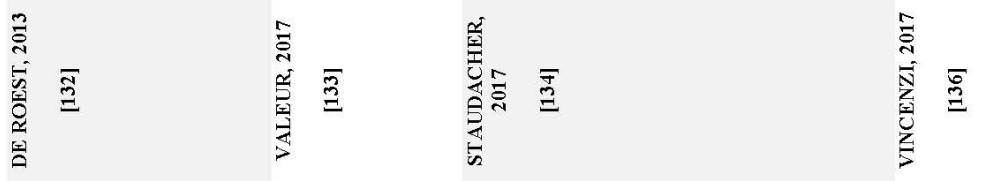



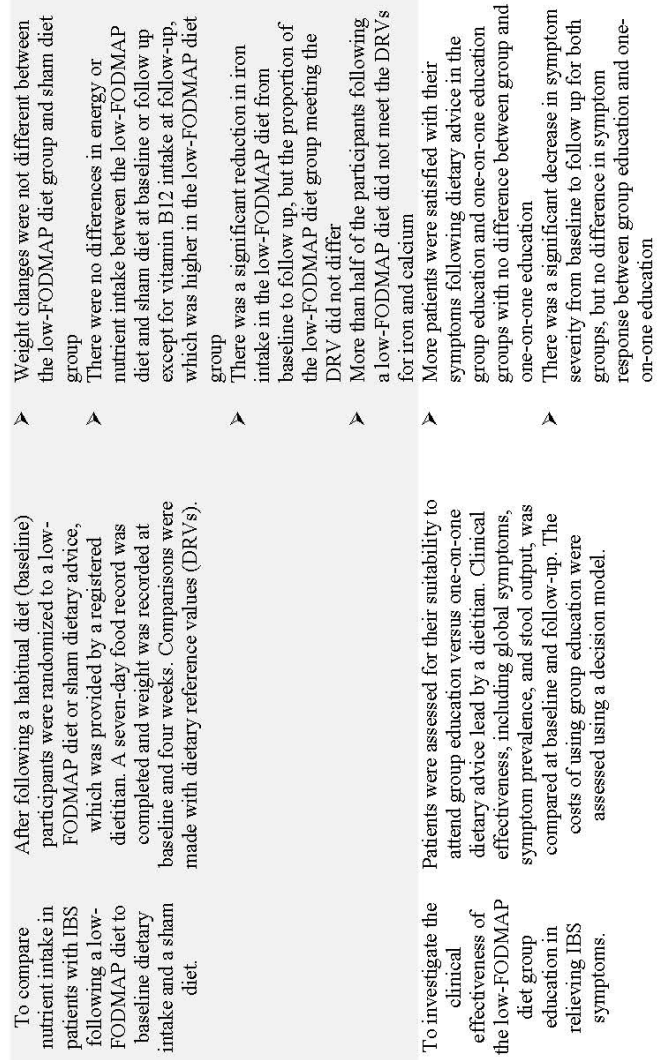

$\alpha$

总

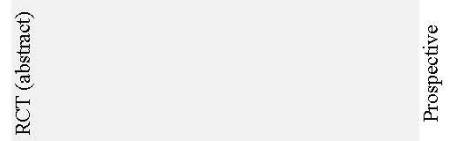

函

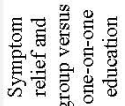
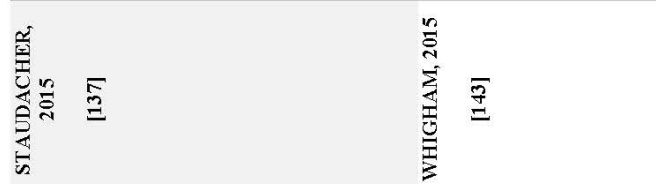

\section{...Continued}


FIRST AUTHOR, YEAR

STAUDACHER, 2011

[10]

BOHN, 2015

[126]

O'KEEFFE, 2017

[131]

DE ROEST, 2013

[132]

STAUDACHER, 2017

[134]

WHIGHAM, 2015

[143]
MEASURE OF COMPLIANCE

A subgroup was asked about their level of compliance at the time of symptom improvement.

Evaluated food diaries to assess compliance to the diet. Four-day food diaries were completed during the screening period and at the final week of the four-week intervention.

Patients recorded their compliance to long term FODMAP restriction using a 4point Likert scale: "continued strict lowFODMAP diet," "reintroduced high-

FODMAP foods to tolerance," "continued the low-FODMAP diet 50\% of the time," "returned to habitual diet." To assess compliance subjects answered questions regarding taste, price, satisfaction, dietary advice, breath tests, and ease of the diet. Participation was

voluntary and data was reported anonymously.

Compliance was self-reported weekly during telephone calls using compliance categories: rarely ( $<25 \%$ of the time), sometimes $(25 \%-50 \%$ of the time), frequently (51\%-75\% of the time), or always ( $76 \%-100 \%$ of the time).

To assess compliance, patients were asked at follow up about the percentage of days they were compliant to the diet during the intervention.

Table 2. Methods of Measuring Compliance in Low-FODMAP Diet Studies 


\section{Chapter 3: Methodology}

The low-FODMAP diet is a therapeutic approach for IBS that reduces the intake of fermentable carbohydrates. This diet has shown to improve symptoms of IBS, however, there is still inadequate evidence for the establishment of firm clinical guidelines. Through the utilization of existing materials on the low-FODMAP diet, researchers in this study instructed patients and observed outcomes related to nutritional intake and speed of symptom improvement. Data in these areas are necessary to ensure nutritional adequacy and provide the least restrictive diet possible. This was accomplished through the following research questions:

1. Are there differences in nutritional intake before and after initiation of the lowFODMAP diet?

2. When compared to reference standards, is nutrient intake adequate after two weeks of the low-FODMAP diet elimination phase?

3. What is the optimal duration for the elimination phase of the low-FODMAP diet to promote symptom relief?

These research questions were answered through a prospective study design using a sample of convenience. The Ohio State University Institutional Review Board approved this study on November 2, 2016 (IRB\# 2016H0320). 


\section{Recruitment}

Individuals diagnosed with IBS who had been referred to The Ohio State University Medical Center's Division of Gastroenterology, Hepatology, and Nutrition, for a consultation with a RDN, were scheduled to attend a group education class for the low FODMAP diet. Inclusion criteria included: age $>18$ years, ability to read English, and a diagnosis of IBS and referral for nutrition consultation/class attendance from the medical provider. Exclusion criteria included inability to provide informed consent for

the study (including prisoners) and pregnancy. After the educational portion of the class, participants were informed of the study. The consent and HIPPA policy for the study were explained to willing participants as a group. Informed consent to take part in the study and agreement to HIPPA terms were completed at that time. A total of 15 males and 55 females attended the class and 35 individuals were consented to participate in the study.

\section{Intervention}

\section{Study Visit}

Participants attended an hour-long class with instructions on the low-FODMAP diet. The information provided through the class was based on the current literature. The class outline focused on the proper delivery of the low-FODMAP diet and consisted of a lecture and participant interaction for diet and menu planning with a question and answer session. The lecture discussed theories behind the role of diet in IBS, how the lowFODMAP diet can relieve IBS symptoms, what the low-FODMAP diet entails, how the low-FODMAP diet should be executed (instruction regarding the difference between 
elimination and reintroduction phases). Information learned through the lecture was followed by practical application and problem solving activities via discussions and the use of visual examples. Kate Scarlata, RDN, LD created the educational materials used in the class PowerPoint and for participants' binders to take home as a resource. Participants received a notebook of educational materials and copies of study instruments (see appendices B-F).

\section{Instructions for Intervention}

Participants were instructed to follow the low-FODMAP diet elimination phase for six weeks. Dates for initiation of the diet were provided to each participant along with steps for documentation of symptoms and dietary intake. Figure 1 provides a depiction of the study intervention.

\section{Health Questionnaire}

The health questionnaire designed by the study researchers was utilized to gather the baseline demographic and health information. This questionnaire specifically focused on information relating to the diagnosis of IBS, and history of nutritional, medical, or other alternative interventions used to relieve symptoms.

\section{IBS-Symptom Severity Score (IBS-SSS)}

This study utilized the validated IBS-Symptom Severity Score (IBS-SSS). The IBS-SSS, developed and validated by Francis and Whorwell, ${ }^{33}$ evaluates individuals using a maximum achievable score of 500, and classifies participants as mild, moderate, or severe based on the score. Answers are self-administered by participants on a visual analog scale consisting of five questions (100 points possible per question). This assessment tool incorporates pain, distension, bowel dysfunction, and quality of life. The 
IBS-SSS is the most common method for assessing symptom severity in research and clinical practice. For this study, the IBS-SSS assisted researchers in determining baseline symptom severity and potential changes for each participant prior to, during, and after the study intervention. Since varying definitions of symptom improvement have impeded the true determination of efficacy of the low FODMAP diet, this study utilized the IBS-SSS measurement tool to define symptom improvement. In the validation of the IBS-SSS, symptom severity classification is described to standardize severity description across practitioners. This study followed the classifications used in the validation paper, which are $<75$ points for remission, $75-175$ for mild IBS, $175-300$ for moderate IBS, and $>300$ for severe IBS. ${ }^{33}$ Symptom improvement for this study was defined as reduction of symptoms $\geq 50$ points, which indicates clinical improvement according to the validation paper. ${ }^{33}$ The IBS-SSS has been used in many studies, including several RCTs and crossover designs. ${ }^{126,129,127,134}$

\section{The Bristol Stool Form Scale}

The Bristol Stool Form Scale developed by Lewis and Heaton ${ }^{32}$ classifies stool into seven different categories based on the form of the stool. This validated tool is commonly used in clinical practice as a description scale for the assessment of stool consistency. The Bristol Stool Form Scale can be used to assist in the subtyping of IBS. ${ }^{1}$ The scale was included with all IBS-SSS forms filled out prior to the diet and during the intervention period.

\section{Three-Day Diet Record}

The use of three-day diet records as a dietary assessment tool has been validated consistently through the literature. ${ }^{138}$ The use of journals to document dietary intake was 
also evaluated by Zia et al. ${ }^{139}$ and does not appear to demonstrate an impact on GI symptoms, although some participants were able to identify individualized relationships between GI symptoms and a meal nutrient through this utilization of meal and symptom journals. Food journals were found to be feasible, useable, and clinically useful for study subjects. Similar results were seen with paper and electronic journals, proving that they are likely both reliable and accurate. ${ }^{139}$ Three-day diet records evaluate dietary data over a shorter period of time, which makes them appropriate for the purpose of this study. Other common dietary assessment tools include the food frequency questionnaire (FFQ) and 24-recall. An FFQ is used to obtain frequency and portion size information about certain listed foods over a period of time. ${ }^{140}$ This tool is typically used to assess certain aspects of the diet, but this study aimed to evaluate all dietary intake. A 24-hour diet recall is useful in assessing dietary intake and other aspects of the diet, but it is unable to account for day-to-day variations in the diet, making it not as appropriate for this study's aim. $^{141}$

Participants were asked to complete the three-day diet record on non-consecutive days to avoid attrition while accounting for day-to-day variations in dietary intake. This study aimed to estimate mean intake, therefore, a pre- and post- low-FODMAP diet initiation three-day diet record was adequate. It is generally recommended to not use a three-day diet record when evaluating changes in an individual's diet after an intervention. ${ }^{142}$ This is because there could be transient changes in behavior that occur as a response to keeping the three-day diet record, known as reactivity. ${ }^{142}$ However, this recommendation does not apply to this study because the diet record assessed nutrient intake during the elimination phase of the low-FODMAP diet, which is only intended for 
short-term adoption before the reintroduction phase of the low-FODMAP diet begins. The literature has found that subjects can find rapid improvement after following the elimination phase of the low-FODMAP diet. ${ }^{10}$ Therefore, compliance to the diet is less likely to be due to reactivity. The use of three-day diet records has been documented in other studies on the low-FODMAP diet, including RCTs. ${ }^{125}$

\section{High-FODMAP Foods Checklist}

To evaluate patients' compliance to the low-FODMAP diet, a high-FODMAP checklist was provided for each day of the six-week intervention period. The highFODMAP checklist contained all dietary choices that participants were instructed to avoid, and participants used it to indicate any of these items consumed during the elimination phase if such incidence occurred.

\section{Additional Data Collection Sources}

\section{Class Evaluation}

A questionnaire was completed at the end of each class to evaluate participants' satisfaction. The results can help evaluate the success of a group education approach to provide dietary interventions. The form created for this study was revised from a satisfaction questionnaire created by Whigham et al., ${ }^{143}$ who also trialed a group approach to deliver low-FODMAP diet education to IBS patients. Table 3 highlights the methodology of this study's instrumentation and data collection.

\section{Phone Communication}

In the case of questions regarding responses on any of the data collection sources utilized in this study, information was confirmed via phone communication (calls/texts). 


\section{Data Analysis}

Data on dietary intake collected using the three-day diet records was analyzed using the Nutrition Data System for Research software (NDSR), version 2016, developed by the Nutrition Coordinating Center (NCC) at the University of Minnesota in Minneapolis, MN. Trained professionals at The Ohio State University in the Clinical Research Center's (CRC) Nutrition Research Laboratory entered dietary intake data. Quality assurance procedures per NDSR protocol were executed, including review of the data by three different individuals for consistency in interpretation and verifying questionable data with the study participant. To summarize the dietary intake data, means and standard deviations were reported for continuous variables. This included percentages of kilocalories from carbohydrates, fat, protein, and percent of RDA for micronutrient intake. Paired samples t-tests were used to assess changes in nutritional intake from baseline to week two based on data from the three-day diet records. An ANOVA with repeated measures was used to compare differences in continuous measures of symptoms measured by the IBS-SSS. A Bonferroni correction was applied for multiple comparisons. Independent samples t-tests were used to assess demographic differences between study participants and non-participants. Frequencies and percentages were used to summarize categorical data. Results were presented as point estimates with $95 \%$ confidence intervals and significance was defined as a two-sided p-value of less than 0.05 . 


\section{Risk to Participants}

The only potential risk to participants involved in this study was a loss of privacy through the review of patients' electronic medical records (EMR).

\section{Confidentiality Concerns}

Participation in this study was completely voluntary, and participants were given the right to withdraw at any time. Participants were assigned a unique study ID number upon enrollment in the study. Each unique study ID number and corresponding MRN was stored in an encrypted electronic master list on a password-protected OSU network drive. Consent forms, HIPPA forms, and all other study information was saved electronically in a similar fashion. All hard copies of study documents were protected in a locked office, accessible only by the PI in the Division of Medical Dietetics (Atwell Hall, Room number 206). All data will be stored for a minimum of three years. 


\begin{tabular}{|c|c|c|c|}
\hline $\begin{array}{c}\text { Instrument/Data } \\
\text { Source }\end{array}$ & Collection & Objective & Analysis \\
\hline $\begin{array}{c}\text { Demographic/health } \\
\text { questionnaire }\end{array}$ & $\begin{array}{l}\text { Before diet } \\
\text { initiation }\end{array}$ & $\begin{array}{c}\text { To understand } \\
\text { baseline and health } \\
\text { information regarding } \\
\text { IBS diagnosis }\end{array}$ & $\begin{array}{c}\text { Descriptive statistics: } \\
\text { mean, standard } \\
\text { deviation (SD) } \\
\text { Inferential statistics: } \\
\text { independent samples } \\
\text { t-test }\end{array}$ \\
\hline $\begin{array}{l}\text { Three-day diet } \\
\text { record }\end{array}$ & $\begin{array}{l}\text { Before diet } \\
\text { initiation, during } \\
\text { week two }\end{array}$ & $\begin{array}{l}\text { To evaluate adequacy } \\
\text { of participant's intake } \\
\text { before and after } \\
\text { initiation of the low- } \\
\text { FODMAP diet }\end{array}$ & $\begin{array}{l}\text { Descriptive statistics: } \\
\text { mean, standard } \\
\text { deviation (SD) } \\
\text { Inferential statistics: } \\
\text { paired samples t-test }\end{array}$ \\
\hline $\begin{array}{c}\text { IBS-Symptom } \\
\text { Severity Scale (IBS- } \\
\text { SSS)/Bristol Stool } \\
\text { Form Scale }\end{array}$ & $\begin{array}{c}\text { Before diet } \\
\text { initiation, during } \\
\text { week two, four, and } \\
\text { six }\end{array}$ & $\begin{array}{l}\text { To evaluate symptom } \\
\text { severity and speed of } \\
\text { symptom } \\
\text { improvement }\end{array}$ & $\begin{array}{l}\text { Descriptive statistics: } \\
\text { mean, standard } \\
\text { deviation (SD) } \\
\text { Inferential statistics: } \\
\text { ANOVA with } \\
\text { repeated measures }\end{array}$ \\
\hline $\begin{array}{c}\text { High-FODMAP } \\
\text { checklist }\end{array}$ & $\begin{array}{l}\text { If high-FODMAP } \\
\text { food is consumed } \\
\text { on any day during } \\
\text { six-week } \\
\text { elimination phase }\end{array}$ & $\begin{array}{c}\text { To measure } \\
\text { compliance to the } \\
\text { low-FODMAP diet }\end{array}$ & $\begin{array}{l}\text { Frequency, } \\
\text { percentages }\end{array}$ \\
\hline Class evaluation & $\begin{array}{c}\text { After low- } \\
\text { FODMAP } \\
\text { education class }\end{array}$ & $\begin{array}{c}\text { To evaluate } \\
\text { participants' } \\
\text { satisfaction with the } \\
\text { low-FODMAP class }\end{array}$ & $\begin{array}{l}\text { Frequency, } \\
\text { percentages }\end{array}$ \\
\hline
\end{tabular}

Table 3. Summary of Study Instrumentation and Additional Data Collection Sources 


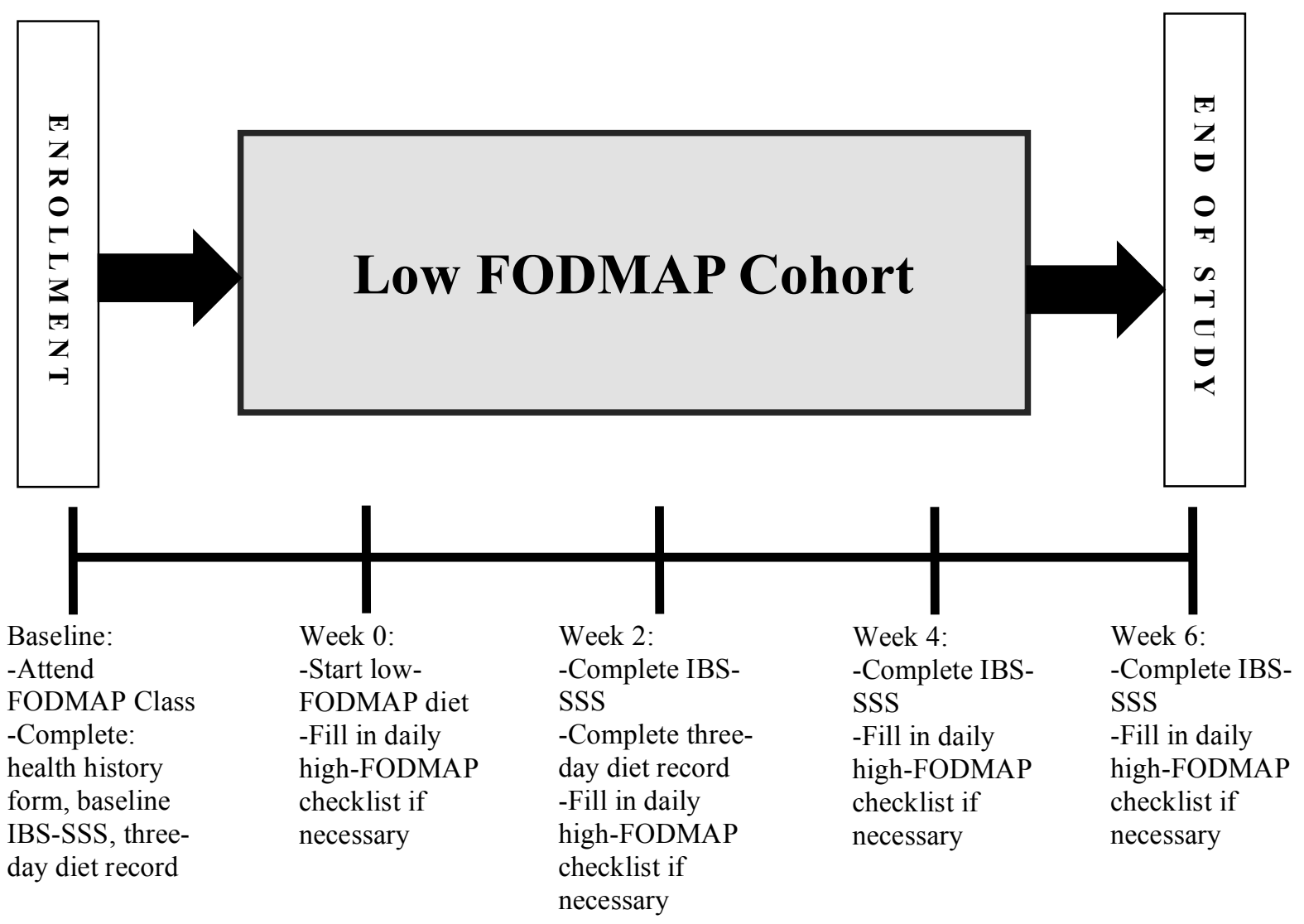

Figure 1. Summary of Study Intervention 


\section{Chapter 4: Results and Discussion}

\section{Results}

\section{Class Attendance and Study Enrollment}

Low-FODMAP diet education classes were held monthly from May 2017-

November 2017. A class was also held in January 2017 from the previous pilot study conducted. Seventy patients attended the low-FODMAP education class, and $35(50 \%)$ enrolled in the study. A display of study enrollment is depicted in figure 2 .

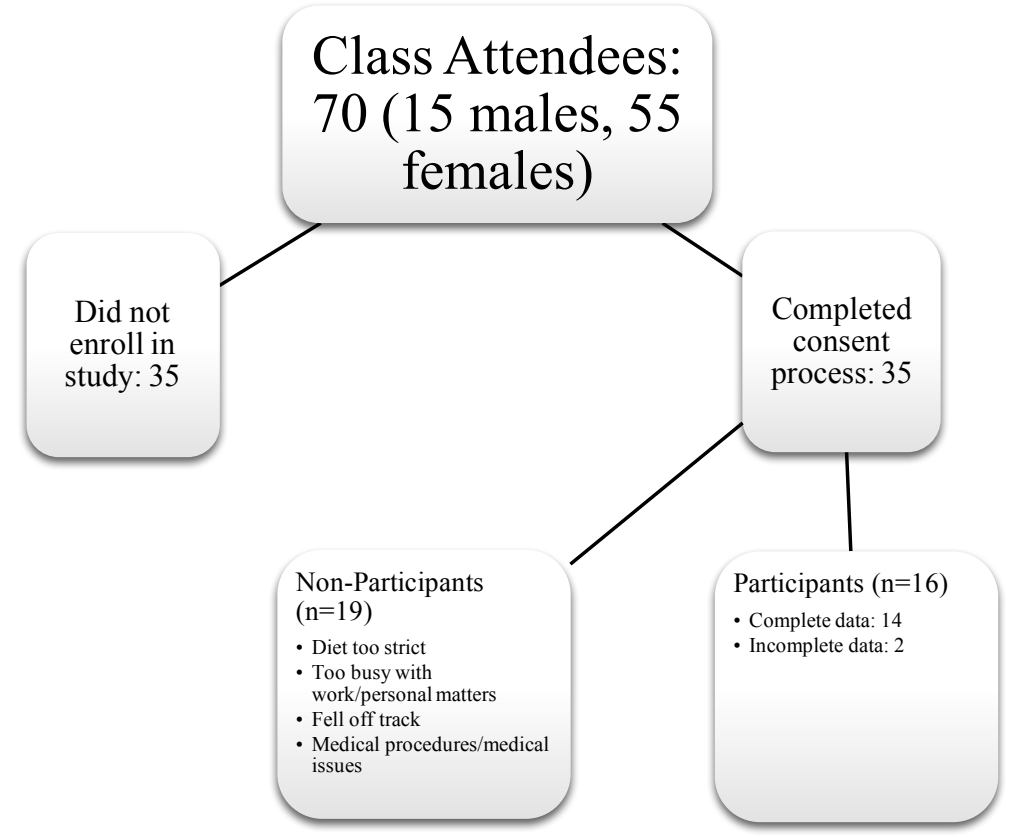

Figure 2. Low-FODMAP Diet Education Class Attendance and Study Enrollment 
There were more females than males who attended our low-FODMAP diet education class. Specifically, $78.5 \%$ of the attendees were females. This is consistent with the diagnosis of IBS as it is more prevalent in females versus males.

\section{Demographics and Health Questionnaire}

General and Diagnosis Information

A total of 35 IBS patients enrolled in this study and completed the consenting process, 16 of which returned the required information during participation in the study. Descriptive information for all consented IBS patients is presented in the following section. Table 4 displays demographic characteristics of our population.

\begin{tabular}{|c|c|c|}
\hline Characteristic & Total $(n=35)$ & Participants $(n=16)$ \\
\hline Age (years), mean, SD & $47.7,16.3$ & $44.1,15.7$ \\
\hline Age range (years) & $21-74$ & $21-70$ \\
\hline \multicolumn{3}{|l|}{ Sex } \\
\hline Females, frequency, $\%$ & 27,77 & $10,62.5$ \\
\hline Males, frequency, \% & 8,23 & $6,37.5$ \\
\hline Body Mass Index $\left(\mathrm{kg} / \mathrm{m}^{2}\right)$, mean, SD & $28.7,8.0$ & $28.7,9.2$ \\
\hline BMI range $\left(\mathrm{kg} / \mathrm{m}^{2}\right)$ & $16.9-54.6$ & $18.3-54.6$ \\
\hline Physically Active, \% & 80 & 75 \\
\hline Smoker, \% & 8.5 & 0 \\
\hline Alcohol Use, $\%$ & 43 & 50 \\
\hline
\end{tabular}

Table 4. Demographic Information of Subjects with Irritable Bowel Syndrome

Twenty-seven of the consenting IBS patients were females and 8 were males. The mean age of this IBS patient population was $47.7 \pm 16.3$ years. There was no statistically significant difference between age for participants $(n=16)$ versus non-participants $(n=19)$. 
The mean BMI was $28.7 \pm 8 \mathrm{~kg} / \mathrm{m}^{2}$, which is categorized as overweight. There was no statistically significant difference between BMI for participants versus non-participants. Twenty-eight IBS patients reported participating in some form of physical activity. Three IBS patients reported smoking and 15 reported a mild intake of alcohol (less than 7 drinks/week or 14 drinks/month).

\section{Lifestyle Factors}

Responses within the health questionnaire identified lifestyle factors that may influence IBS symptoms or severity and the relationship to dietary intake. In this population, 13 IBS patients previously received nutrition therapy for their IBS from a healthcare provider or RDN. Of these 13 IBS patients, 5 specified receiving information about the low-FODMAP diet. Thirteen IBS patients reported to be currently following a special diet: 6 were following an elimination diet, 1 was following a low-FODMAP diet, 1 was following the National Institute for Health and Care Excellence NICE) diet, 1 was following a low-sugar diet, 1 was following a Meal Replacement Program (MRP)/Small Intestinal Bowel Overgrowth (SIBO) diet, 1 was following a liquid/pureed diet, and 2 individuals did not list the diet they were following.

Twenty-two IBS patients claimed to be excluding at least some foods. Of these, the most common foods to exclude were all or some dairy, fruit, vegetables and meats. Some of the dietary choices already being excluded by IBS patients were foods containing FODMAPs. Figure 3 provides a breakdown of the percentage of IBS patients excluding certain FODMAP groups. 


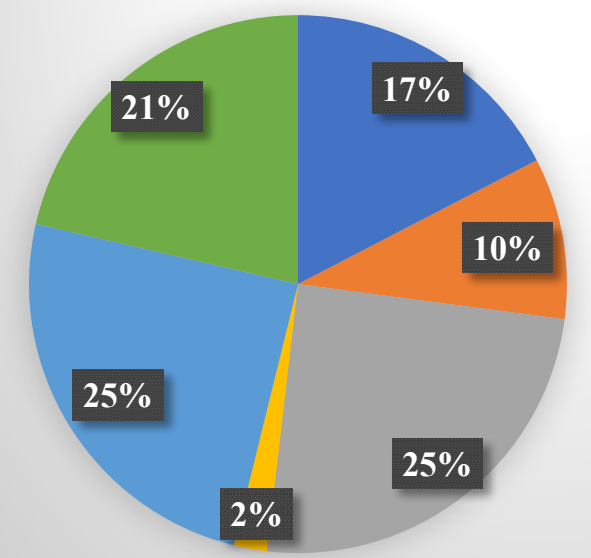

Lactose

Fructose

Fructans/GOS

Polyols

- Fructose, Fructans/GOS, Polyols

- Low-FODMAP

Figure 3. FODMAP Food Groups Restricted Prior to Low-FODMAP Education

Most of the foods that IBS patients were excluding contained one or more

\section{FODMAPs.}

\section{Medication and Therapy Usage}

Prescription medications were utilized by 17 IBS patients. Figure 4 provides a summary of the prescription treatments utilized by IBS patients in this study.

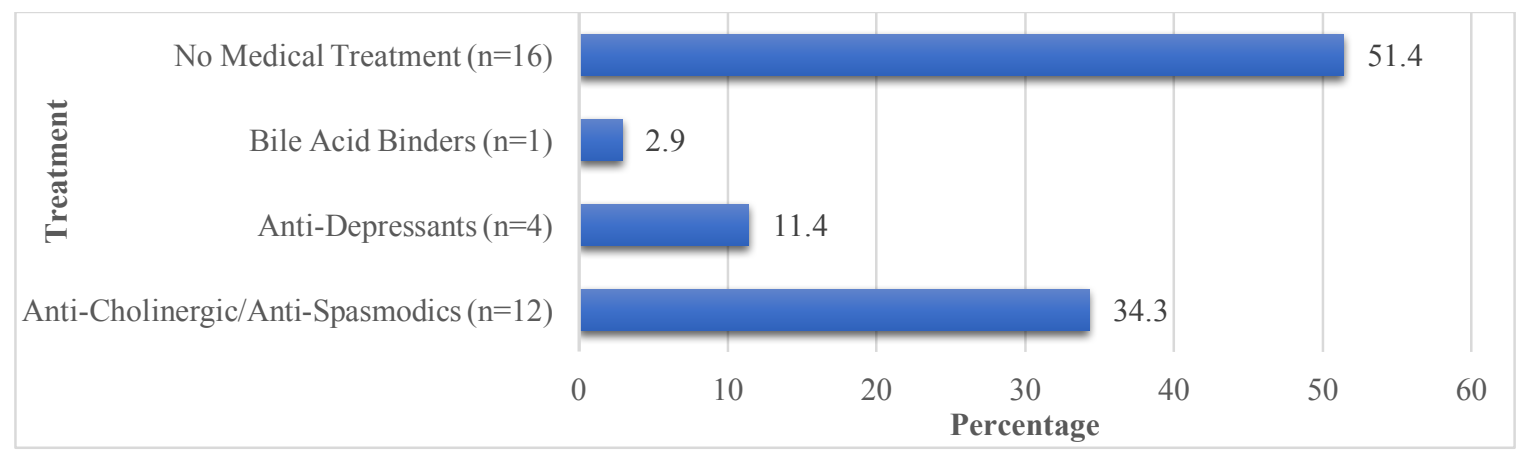

Figure 4. Prescription Treatments Utilized by All Patients with IBS (\%) $(n=35)$ 
Non-prescription medications were utilized by 23 IBS patients. Figure 5 provides a summary of the non-prescription treatments utilized by IBS patients in this study.

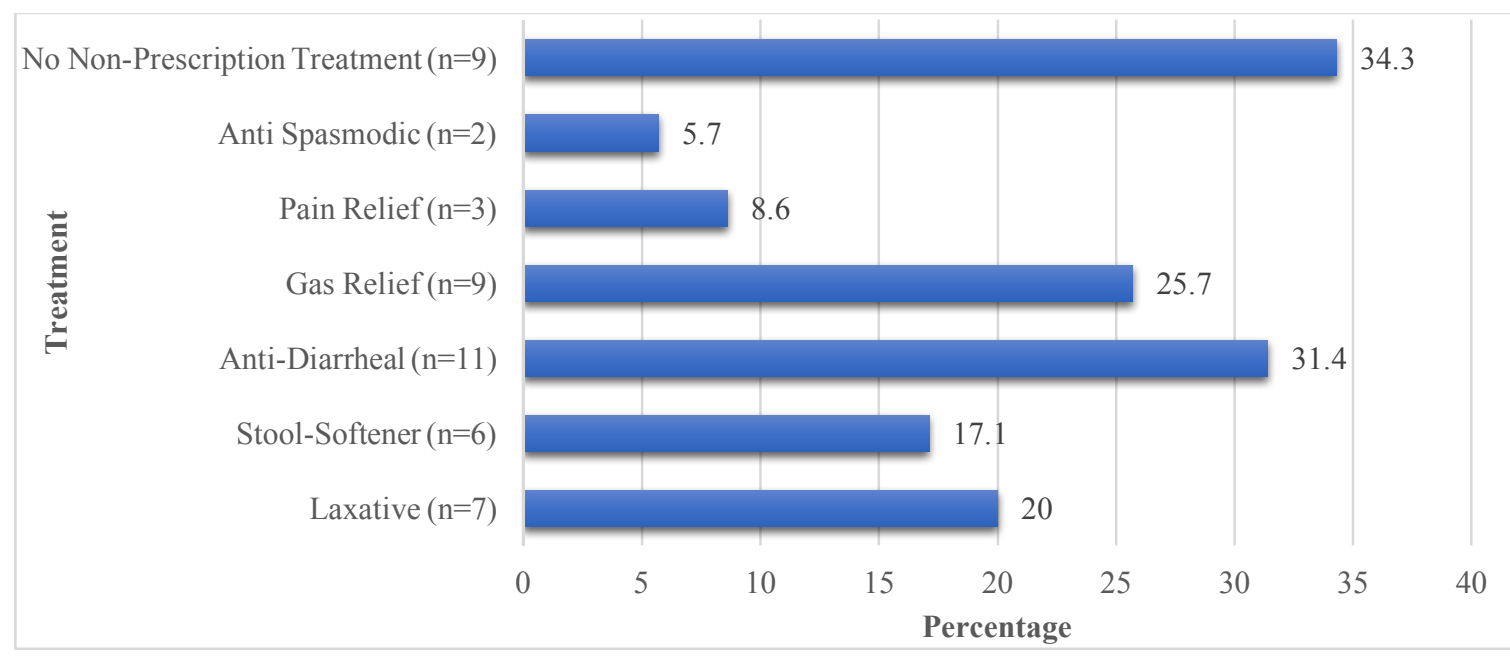

Figure 5. Non-Prescription Treatments Utilized by All Patients with IBS (\%) (n=35)

Vitamin, mineral, or nutritional supplements were used by 28 IBS patients. There were 10 and 6 IBS patients utilizing herbal supplements and alternative therapies, respectively.

\section{Bristol Stool Form Scale, IBS-SSS and High-FODMAP Checklists}

\section{IBS Diagnosis Information}

Data to assess symptom severity was returned by 16 participants and information reported below reflects data from these participants. The Bristol Stool Form Scale can be used to categorize individuals based on their IBS subtype. If an individual is characterized by a 1 or 2 on the Bristol Stool Form Scale, they are categorized as IBS-C. 
Individuals that are a 6 or 7 on the Bristol Stool Form Scale are categorized as IBS-D. If an individual is anywhere between 3-5 on the Bristol Stool Form Scale, they are categorized as IBS-U, while individuals who fall into multiple categorizes as classified as IBS-M. An image of the Bristol Stool Form Scale is provided in Appendix C. Figure 6 displays the subtypes of IBS patients based on their Bristol Stool Form Scale results.

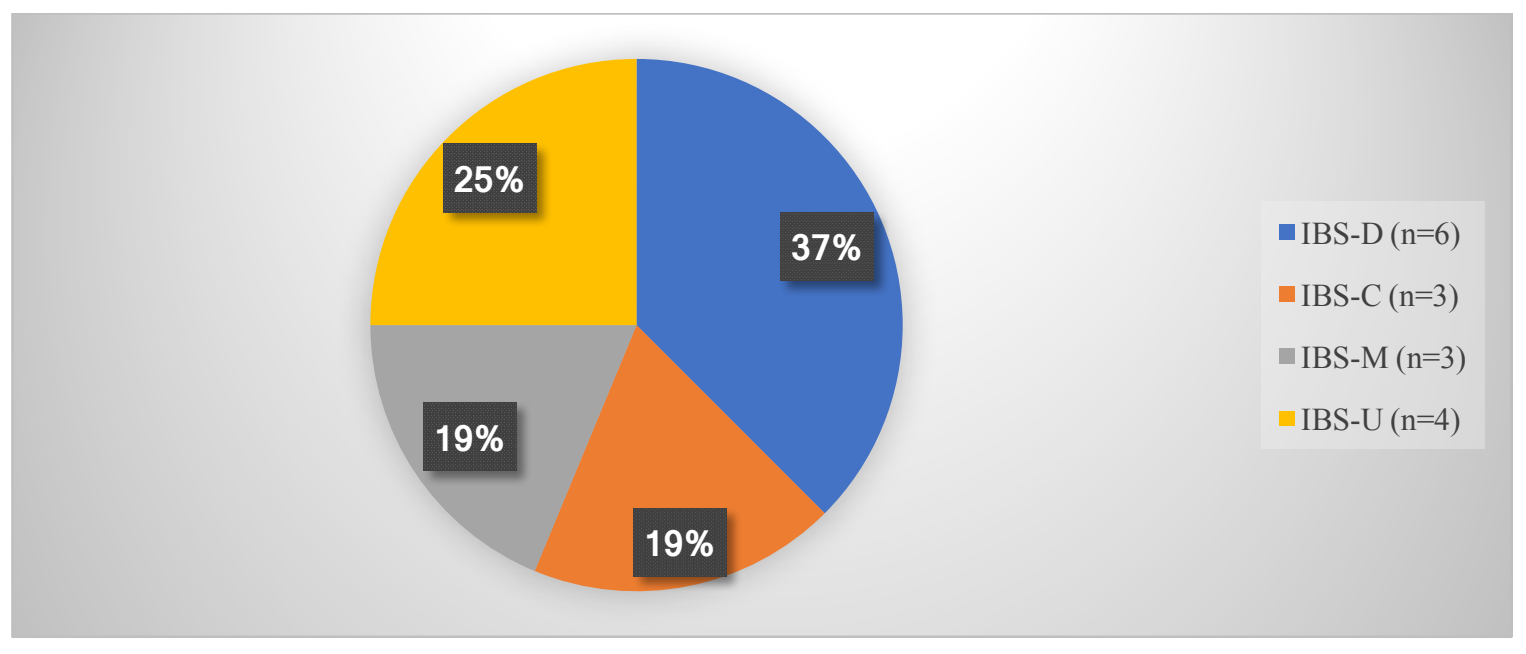

Figure 6. IBS Diagnosis Based on Subtype According to Bristol Stool Form Scale Responses $(n=16)$

IBS-SSS questionnaires were completed at baseline, week 2, week 4, and week 6 by study participants. To measure compliance, participants were also instructed to complete a high-FODMAP checklist on any day they consumed a high-FODMAP food. Table 5 displays each individual participant's responses to these study instruments. 


\begin{tabular}{|c|c|c|c|c|c|c|c|c|}
\hline $\begin{array}{c}\text { Study ID } \\
(n=16)\end{array}$ & $\begin{array}{l}\text { Baselin } \\
\text { e Score }\end{array}$ & $\begin{array}{c}\text { Wee } \\
\text { k } 2 \\
\text { Scor } \\
\text { e }\end{array}$ & $\begin{array}{c}\text { Noncomplianc } \\
\text { e for Week } 2 \\
\text { (\# of days) }\end{array}$ & $\begin{array}{c}\text { Wee } \\
\text { k } 4 \\
\text { Scor } \\
\text { e }\end{array}$ & $\begin{array}{c}\text { Noncomplianc } \\
\text { e for Week } 4 \\
\text { (\# of days) }\end{array}$ & $\begin{array}{c}\text { Wee } \\
\text { k } 6 \\
\text { Scor } \\
\text { e }\end{array}$ & $\begin{array}{c}\text { Noncomplianc } \\
\text { e for Week } 6 \\
\text { (\# of days) }\end{array}$ & $\begin{array}{c}\text { Responder } \\
?(\geq 50- \\
\text { point } \\
\text { reduction) }\end{array}$ \\
\hline $\begin{array}{c}\text { FODMAP00 } \\
2\end{array}$ & 335 & 67 & 0 & 50 & 2 & 191 & 4 & Yes \\
\hline $\begin{array}{c}\text { FODMAP00 } \\
3\end{array}$ & 367 & 205 & 4 & 200 & 3 & 91 & 2 & Yes \\
\hline $\begin{array}{c}\text { FODMAP00 } \\
4\end{array}$ & 434 & 0 & 6 & 0 & 0 & 0 & 0 & Yes \\
\hline $\begin{array}{c}\text { FODMAP00 } \\
7\end{array}$ & 209 & 100 & 0 & 150 & 0 & 66 & 0 & Yes \\
\hline $\begin{array}{c}\text { FODMAP00 } \\
8\end{array}$ & 400 & 192 & 1 & 267 & 0 & 305 & 3 & Yes \\
\hline $\begin{array}{c}\text { FODMAP00 } \\
9\end{array}$ & 235 & 215 & 5 & 305 & 7 & 135 & 12 & Yes \\
\hline $\begin{array}{c}\text { FODMAP01 } \\
2\end{array}$ & 287 & 277 & 0 & 327 & 0 & 302 & 0 & No \\
\hline $\begin{array}{c}\text { FODMAP01 } \\
3\end{array}$ & 384 & 255 & 0 & 125 & 0 & 95 & 0 & Yes \\
\hline $\begin{array}{c}\text { FODMAP01 } \\
5\end{array}$ & 317 & 66 & 1 & 180 & 1 & 160 & 0 & Yes \\
\hline $\begin{array}{c}\text { FODMAP01 } \\
9\end{array}$ & 420 & 225 & 0 & 227 & 0 & 234 & 0 & Yes \\
\hline $\begin{array}{c}\text { FODMAP02 } \\
3\end{array}$ & 299 & 294 & N/A & 267 & N/A & 188 & $\mathrm{~N} / \mathrm{A}$ & Yes \\
\hline $\begin{array}{c}\text { FODMAP03 } \\
1\end{array}$ & 342 & 269 & 0 & 269 & 2 & 269 & 0 & Yes \\
\hline $\begin{array}{c}\text { FODMAP03 } \\
4\end{array}$ & 158 & 66 & 0 & 33 & 2 & 0 & 0 & Yes \\
\hline $\begin{array}{c}\text { FODMAP04 } \\
2\end{array}$ & 275 & 141 & 0 & 131 & 0 & 66 & 0 & Yes \\
\hline $\begin{array}{c}\text { FODMAP04 } \\
6\end{array}$ & 131 & 0 & 0 & 10 & 1 & 0 & 0 & Yes \\
\hline $\begin{array}{c}\text { FODMAP04 } \\
9\end{array}$ & 380 & 329 & 2 & 220 & 0 & 237 & 1 & Yes \\
\hline
\end{tabular}

Table 5. Participant's Response and Compliance to the Low-FODMAP Diet

A responder to the diet was defined as an individual who saw a reduction $\geq 50$ points in IBS-SSS while following the low-FODMAP diet. Compliance to the diet was defined as the total avoidance of FODMAP-containing foods. Fifteen (94\%) participants reported a clinical response (reduction of $\geq 50$ points on IBS-SSS questionnaire) to the low-FODMAP diet based on their IBS-SSS scores over the 6-week study period. For the participants who clinically responded to the low-FODMAP diet $(n=15), 13$ responded 
after two weeks. The other 2 responders saw a clinical response after six weeks of the diet. A reduction in IBS severity (severe, moderate, mild, or remission) was also evaluated to further support participants' response to the low-FODMAP diet. Fourteen out of the 15 responders had a reduction in IBS severity. Twelve of 14 responders had this reduction in severity after two weeks of the low-FODMAP diet. Table 6 displays a summary of the statistical analysis of IBS-SSS data for participants.

\begin{tabular}{|c|c|c|c|c|c|}
\hline Week & $\begin{array}{c}\text { Mean IBS- } \\
\text { SSS Score } \\
\quad(n=16)\end{array}$ & $\begin{array}{l}\text { Standard } \\
\text { Deviation }\end{array}$ & $\begin{array}{c}\text { P-value } \\
\text { from } \\
\text { Baseline }\end{array}$ & $\begin{array}{c}\text { Degrees of } \\
\text { Freedom }\end{array}$ & $\mathbf{F}$ \\
\hline Baseline & 310.8 & 90.8 & & \multirow[t]{4}{*}{3} & \multirow[t]{4}{*}{19.997} \\
\hline Week 2 & 168.8 & 107.0 & $<0.001$ & & \\
\hline Week 4 & 172.6 & 106.4 & 0.002 & & \\
\hline Week 6 & 146.2 & 105.5 & $<0.001$ & & \\
\hline
\end{tabular}

ANOVA with repeated measures indicated that there was a statistically significant difference in IBS-SSS across the six-week time span, $F(3,45)=19.997, \mathrm{p}<0.001$. Followup Bonferroni testing showed a statistically significant difference between baseline and each of the following weeks $(2,4,6=p<0.001)$.

Ten $(63 \%)$ participants reported noncompliance to the diet at least once during the six-week period. One participant did not return high-FODMAP checklists or confirm full compliance to the diet via text/phone therefore, for data analysis this individual was considered to be fully compliant. The highest non-compliance rate was reported from week 4 to week 6 of the diet, with a mean of 1.4 days. From baseline to week 2 and from 
week 2 to week 4, patients reported a mean non-compliance of 1.2 and 1.1 days, respectively. Figure 7 displays the frequency that various high-FODMAP foods were consumed by study participants over the six-week intervention period.

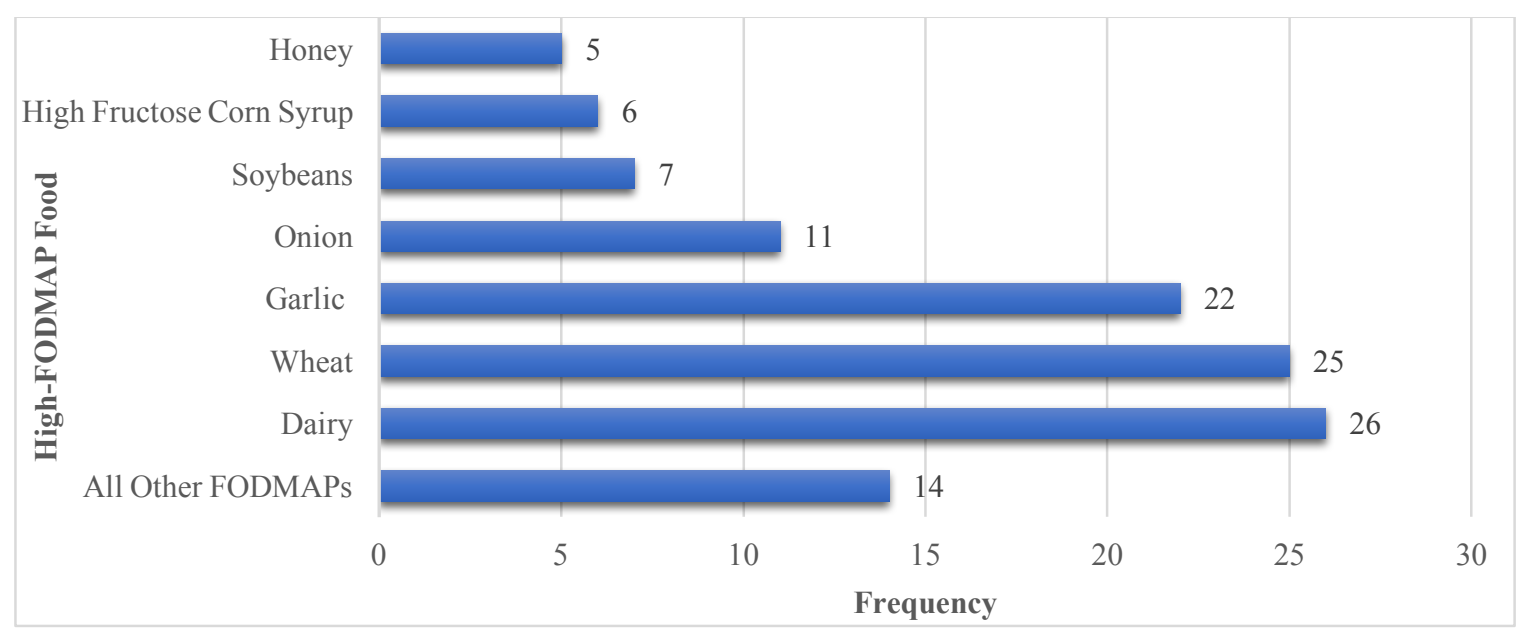

Figure 7. Frequency of High-FODMAP Foods Consumed by Non-Compliant Participants $(n=10)$

Dairy products, wheat, garlic, and onion appear to be the largest contributors to non-compliance during the low-FODMAP diet and were consumed most frequently during the six-week intervention period. Dairy was consumed by 6 participants one or more times during the six-week intervention period, with 4 consuming milk, 3 consuming ice cream, 1 consuming yogurt, and 1 consuming cottage cheese. Wheat was consumed by 5 participants, garlic was consumed by 6 participants, and onion was consumed by 5 participants one or more times during the six-week intervention period. 


\section{Three-Day Diet Records}

Completed three-day diet records were returned by 14 participants. Figure 8 displays the mean change in calories for all participants from baseline to week 2 of the low-FODMAP diet.

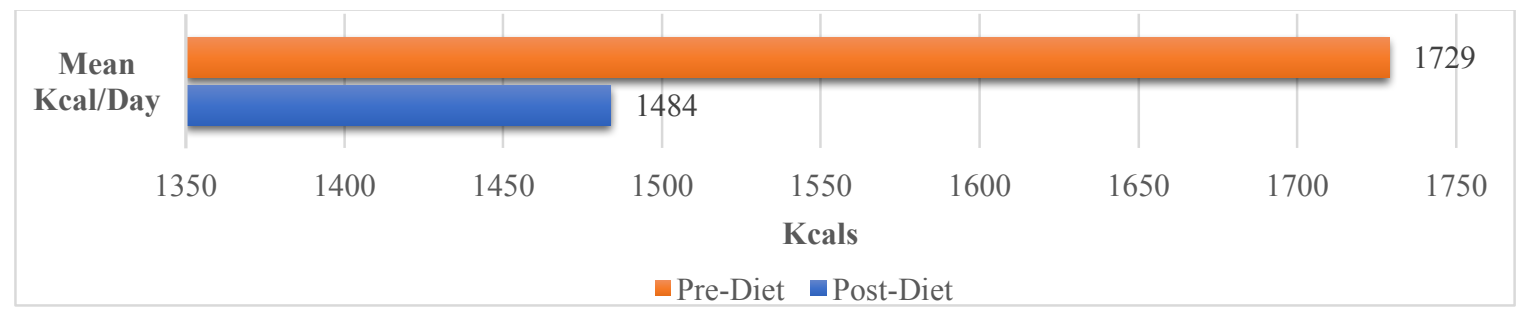

Figure 8. Pre-and Post-Diet Mean Caloric Intake for All Participants(n=14)

Six participants had a reduction in total calories per day when following the lowFODMAP diet, and 50\% (3/6) of these participants had a reduction $>50 \%$ in total calories per day. There were 5 participants who consumed under $1200 \mathrm{kcal} /$ day after initiating the low-FODMAP diet, and 2 of these 5 participants were already consuming under 1200 $\mathrm{kcal} /$ day prior to initiating the diet. Figure 9 displays the mean change in macronutrients for all participants from baseline to week two of the low-FODMAP diet. 


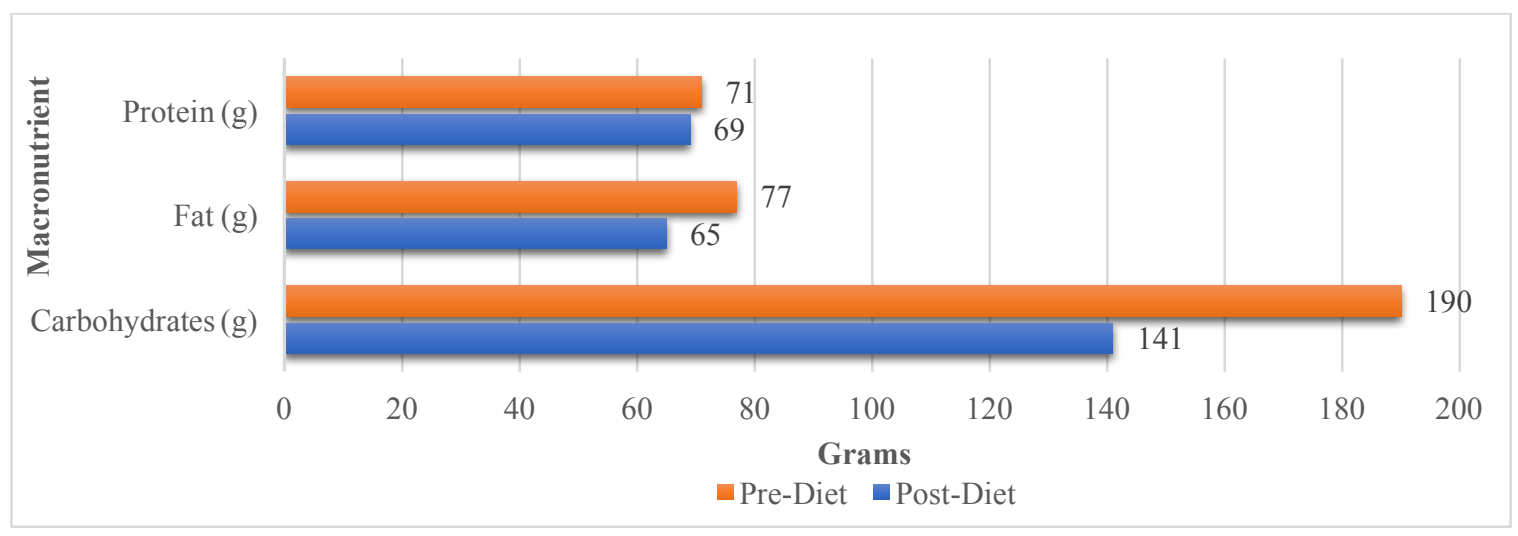

Figure 9. Pre- and Post-Diet Macronutrient Intake for All Participants (n=14).

The main macronutrient of concern after initiating the low-FODMAP diet is carbohydrates, since the diet requires a restriction of certain types. Ten participants had a reduction in carbohydrate intake as a percentage of total energy.

The macronutrient distribution for participants at baseline and week two is displayed in figure 10. Saturated fatty acids (SFA) and added sugars are recommended by the Dietary Guidelines for Americas to be consumed in limited amounts. The contributions of SFA and added sugars to total energy intake is displayed in figure 10 as well. 


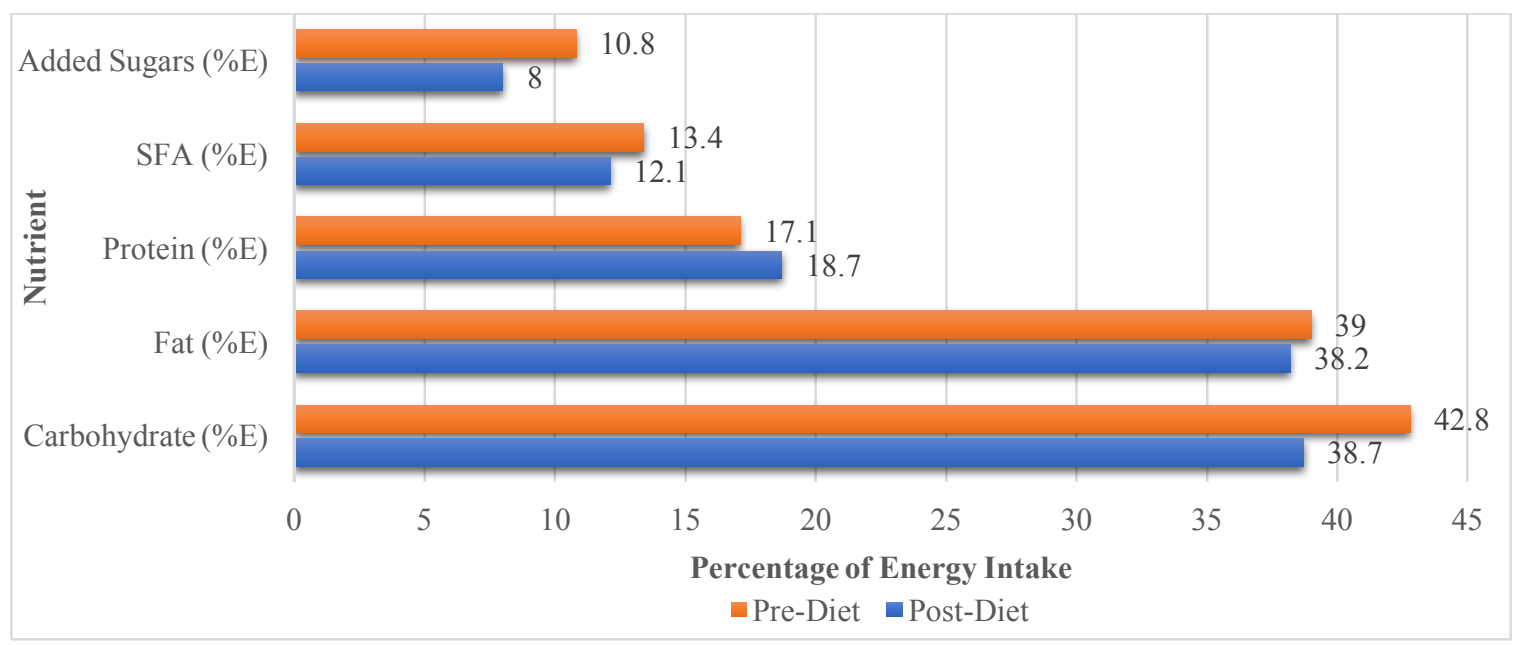

Figure 10. Pre- and Post-Diet Mean Distribution of Energy Intake for All Participants $(n=14)$

At week two of the low-FODMAP diet, there was a mean reduction in carbohydrate, fat, saturated fat, and added sugar consumption for participants. There was a mean increase in protein consumption for participants.

Due to the nature of the low-FODMAP diet, fiber intake could be affected. Figure 11 displays the mean change in fiber intake for all participants from baseline to week two. 


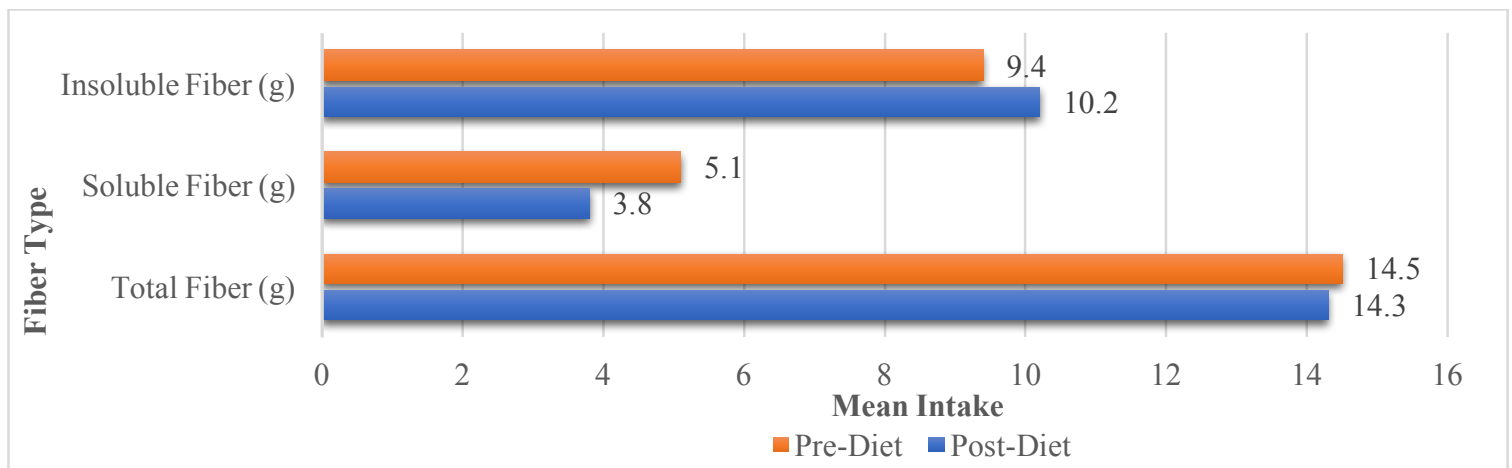

Figure 11. Mean Change in Fiber Intake After Initiation of the Low-FODMAP Diet for All Participants $(n=14)$

Seven participants had a reduction in total fiber intake after starting the lowFODMAP diet. For soluble and insoluble fiber, 11 and 6 participants reduced intake postdiet initiation, respectively. Participants' mean intake of fiber and soluble fiber both decreased after initiating the low-FODMAP diet, while the mean intake of insoluble fiber increased.

The macronutrient distribution as a percentage of total energy intake was compared to the Average Macronutrient Distribution Range (AMDR) set by the Institute of Medicine (IOM). ${ }^{144}$ Data regarding fiber, SFA, and added sugar intake was compared to recommendations from the 2015-2020 Dietary Guidelines for Americans created by the US Department of Health and Human Services (HHS) and US Department of Agriculture (USDA). ${ }^{145}$ Estimated daily energy requirements for weight maintenance were calculated using participant's reported weight and based on $20-25 \mathrm{kcal} / \mathrm{kg} / \mathrm{day}$. Table 6 displays the distribution of participants based on how their dietary intake 
compared to the recommended ranges for these nutrients and their estimated energy requirements per day.

\begin{tabular}{|c|c|c|c|c|}
\hline Nutrient & $\begin{array}{c}\text { Recommended } \\
\text { Range }\end{array}$ & $\begin{array}{c}\text { Met } \\
\text { Recommendation } \\
\text { (n) }\end{array}$ & $\begin{array}{c}\text { Below } \\
\text { Recommendation } \\
\text { (n) }\end{array}$ & $\begin{array}{c}\text { Exceeded } \\
\text { Recommendation } \\
\text { (n) }\end{array}$ \\
\hline $\begin{array}{c}\text { Calories } \\
\text { (kcal/day) }\end{array}$ & Varies & 1 & 8 & 5 \\
\hline $\begin{array}{c}\text { Protein } \\
\text { (\% kcal) }^{2}\end{array}$ & $10-35 \%$ & 13 & 1 & 0 \\
\hline $\begin{array}{c}\text { Carbs } \\
\text { (\%kcal) }\end{array}$ & $45-65 \%$ & 3 & 11 & 0 \\
\hline $\begin{array}{c}\text { Fat } \\
(\% \text { kcal })^{2}\end{array}$ & $20-35 \%$ & 5 & 0 & 9 \\
\hline $\begin{array}{c}\text { Saturated } \\
\text { Fat } \\
\text { (\%kcal) }^{3}\end{array}$ & $<10 \%$ & 6 & N/A & 8 \\
\hline $\begin{array}{c}\text { Added } \\
\text { Sugars } \\
\text { (\%kcal) }^{3}\end{array}$ & $<10 \%$ & 10 & N/A & 4 \\
\hline Fiber (g) & Varies & 1 & 13 & N/A \\
\hline \multicolumn{5}{|c|}{$\begin{array}{l}\text { * Recommendations include the following: } \\
\text { 1. Estimated daily energy requirements for weight maintenance }(20-25 \mathrm{kcal} / \mathrm{kg} / \mathrm{d}) \\
\text { 2. Acceptable Macronutrient Distribution Range (AMDR) set by the Institute of Medicine } \\
\text { (IOM) })^{144} \\
\text { 3. Recommendations from the } 2015-2020 \text { Dietary Guidelines for Americans }{ }^{145}\end{array}$} \\
\hline Table 7. Dist & ibution of Partic & ipants Based on $\mathrm{Pel}$ & ent Nutrient Int & еесоттеп \\
\hline
\end{tabular}

At baseline, 9 participants were already consuming below their estimated energy requirements. After initiating the low-FODMAP diet, 8 participants were below their estimated energy requirements, and 6 of these 8 participants were previously below their requirements at baseline. Two additional participants that were above their estimated 
energy requirements at baseline fell below their requirements after diet initiation. Two participants' intakes increased from below their estimated energy requirements at baseline to above their estimated energy requirements post-diet initiation, and 1 participant previously below estimated energy requirements increased intake to within estimated caloric range.

Eleven participants were below the AMDR for carbohydrates $\left(<45 \%\right.$ of energy ${ }^{144}$ ) after initiating the diet. Eight of these 11 participants were already consuming below the AMDR for carbohydrates prior to beginning the low-FODMAP diet. Many participants were above the recommended ranges for fat and SFA ( 9 and 8 participants, respectively). Seven of the 9 participants above the AMDR for fat $\left(>35 \%\right.$ of energy $\left.{ }^{144}\right)$ were already consuming a higher amount of fat than the AMDR at baseline. All 8 of the participants who were consuming above the recommended range for SFA $\left(>10 \%\right.$ of energy $\left.{ }^{145}\right)$ were also above this recommendation at baseline. There were 5 additional participants consuming above the recommended range for SFA at baseline that fell within the recommendation after initiating the low-FODMAP diet. Protein intake was within the recommended range for almost all participants at baseline and post-diet initiation.

The recommendation for an adequate intake of fiber is 14 grams per 1,000 calories consumed. ${ }^{145}$ At baseline, only 2 participants met this recommendation, and postdiet initiation only 1 participants met this recommendation.

Three specific vitamins were evaluated in this study, vitamin $\mathrm{D}$, vitamin $\mathrm{C}$, and folate (vitamin B9). Two specific minerals were evaluated, calcium and iron. At week two of the low-FODMAP diet, there was a mean reduction in calcium and folate intake for study participants. There was a mean increase in iron, vitamin $\mathrm{D}$, and vitamin $\mathrm{C}$ 
intake after two weeks of the low-FODMAP diet. Figure 12 displays the mean change in vitamin $\mathrm{C}$ intake for all participants from baseline to week two of the low-FODMAP diet.

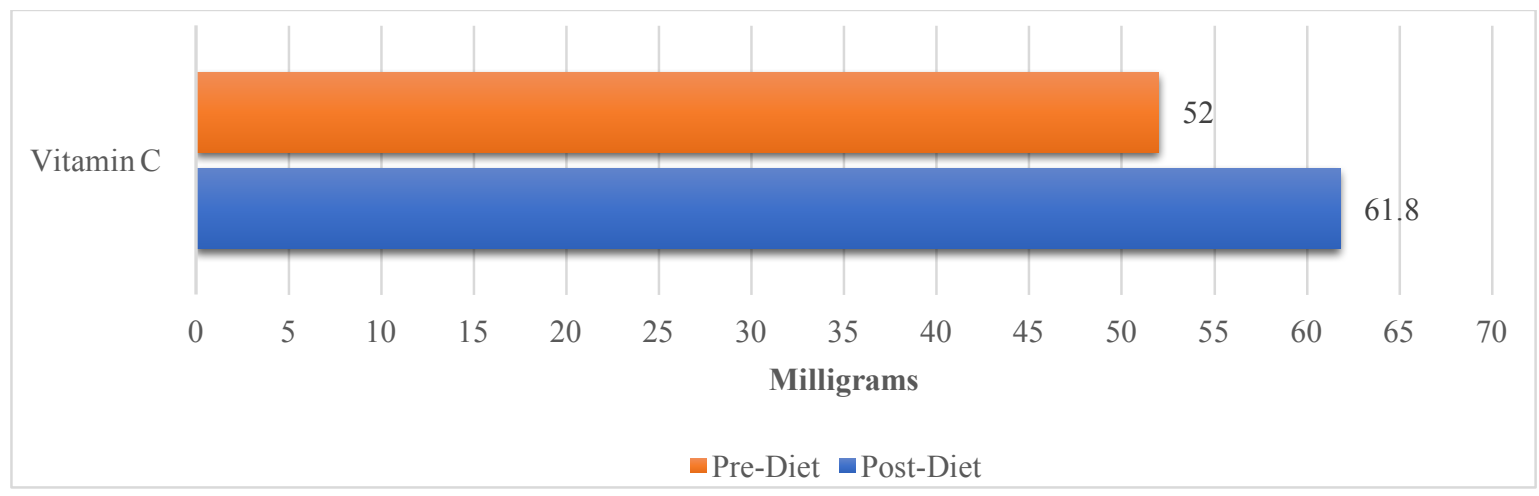

Figure 12. Mean Change in Vitamin C Intake After Initiation of the Low-FODMAP Diet for All Participants $(n=14)$

Vitamin C intake decreased for 7 participants post diet initiation. Twelve participants were not meeting the RDA for vitamin C (90 mg for men and $75 \mathrm{mg}$ for women $^{145}$ ) pre-diet initiation. Post-diet initiation, 1 of these participants increased vitamin $\mathrm{C}$ intake above the RDA; however, the other 11 participants remained below the RDA for vitamin C. Figure 13 displays the mean change in vitamin D intake for all participants from baseline to week two of the low-FODMAP diet. 


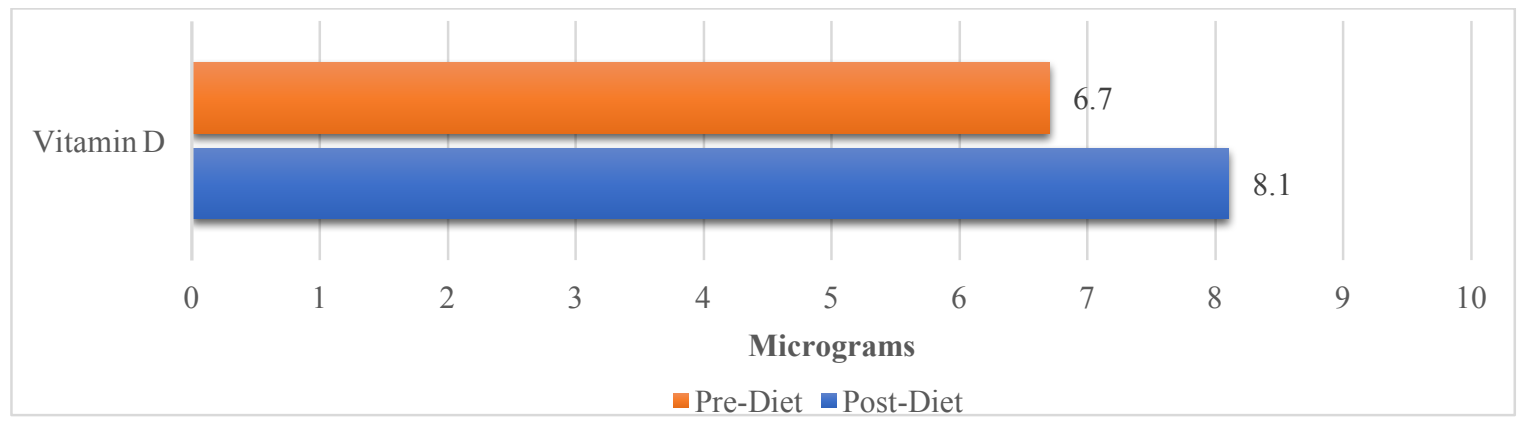

Figure 13. Mean Change in Vitamin D Intake After Initiation of the Low-FODMAP Diet for All Participants $(n=14)$

Regarding vitamin intake, vitamin D intake decreased for 5 participants post initiation of the low-FODMAP diet. Twelve participants were not meeting the RDA for vitamin D (15 mcg for men and women ${ }^{145}$ ) pre-diet initiation, and 1 additional participant's intake fell below the RDA post-diet initiation. Figure 14 displays the mean change in folate intake for all participants from baseline to week two of the lowFODMAP diet.

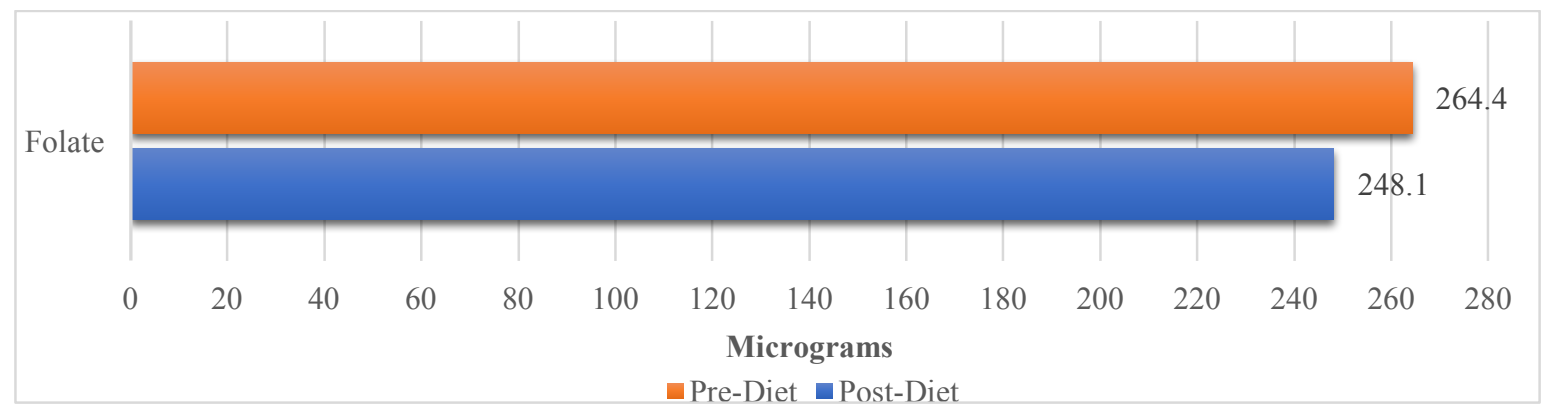

Figure 14. Mean Change in Folate Intake After Initiation of the Low-FODMAP Diet for All Participants $(n=14)$ 
Folate intake decreased for 8 participants post-diet initiation. Thirteen participants were not meeting the RDA for folate $\left(400 \mathrm{mcg}\right.$ for men and women ${ }^{145}$ ) pre-diet initiation. Post diet initiation, 1 of these participants increased folate intake above the RDA; however, the other 12 participants remained below the RDA for folate. Figure 15 displays the mean change in calcium intake for all participants from baseline to week two of the low-FODMAP diet.

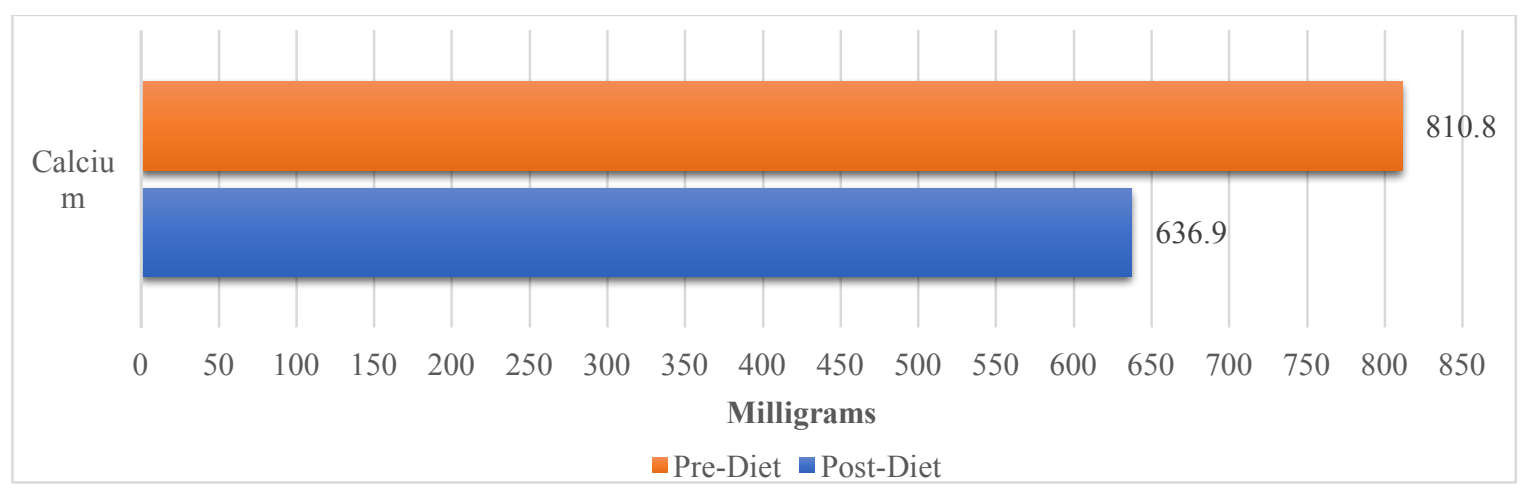

Figure 15. Mean Change in Calcium Intake After Initiation of the Low-FODMAP Diet for All Participants ( $n=14)$

Calcium intake decreased for 10 participants post initiation of the low-FODMAP diet. Ten participants were not meeting the RDA (1000 mg for men and women $\left.{ }^{145}\right)$ for calcium pre-diet initiation, and 3 more participants' intakes fell below the RDA for calcium post-diet initiation. One participant that was previously below the RDA for 
calcium pre-diet initiation did increase calcium intake to above the RDA post-diet initiation. Figure 16 displays the mean change in iron intake for all participants from baseline to week two of the low-FODMAP diet.

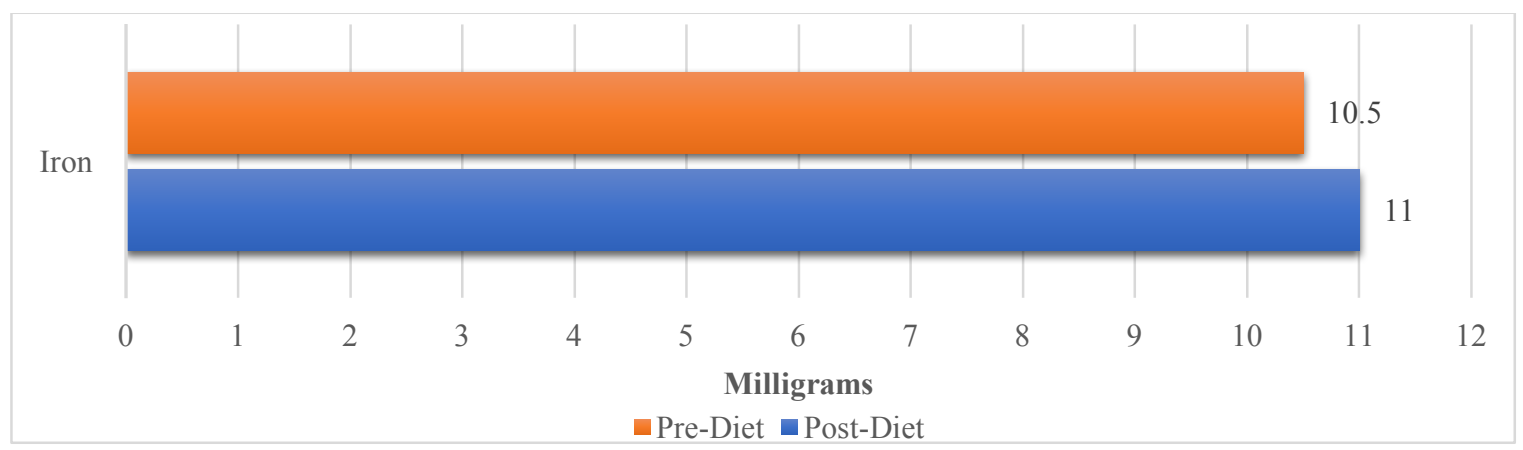

Figure 16. Mean Change in Iron Intake After Initiation of the Low-FODMAP Diet for All Participants $(n=14)$

Iron intake decreased for 4 participants, remained the same for 1 participant, and increased for 9 participants post-diet initiation. Eleven participants were not meeting the RDA ( $8 \mathrm{mg}$ for men and $18 \mathrm{mg}$ for women ${ }^{145}$ ) for iron pre-diet initiation. Two additional participants' intakes fell below the RDA for iron post-diet initiation; however, 2 other participants' intakes increased to above the RDA for iron post-diet initiation.

The low-FODMAP diet is highly restrictive and may require participants to find substitutions for common dietary choices in each food group. The change in servings for each food group was evaluated and figure 17 displays these changes. 


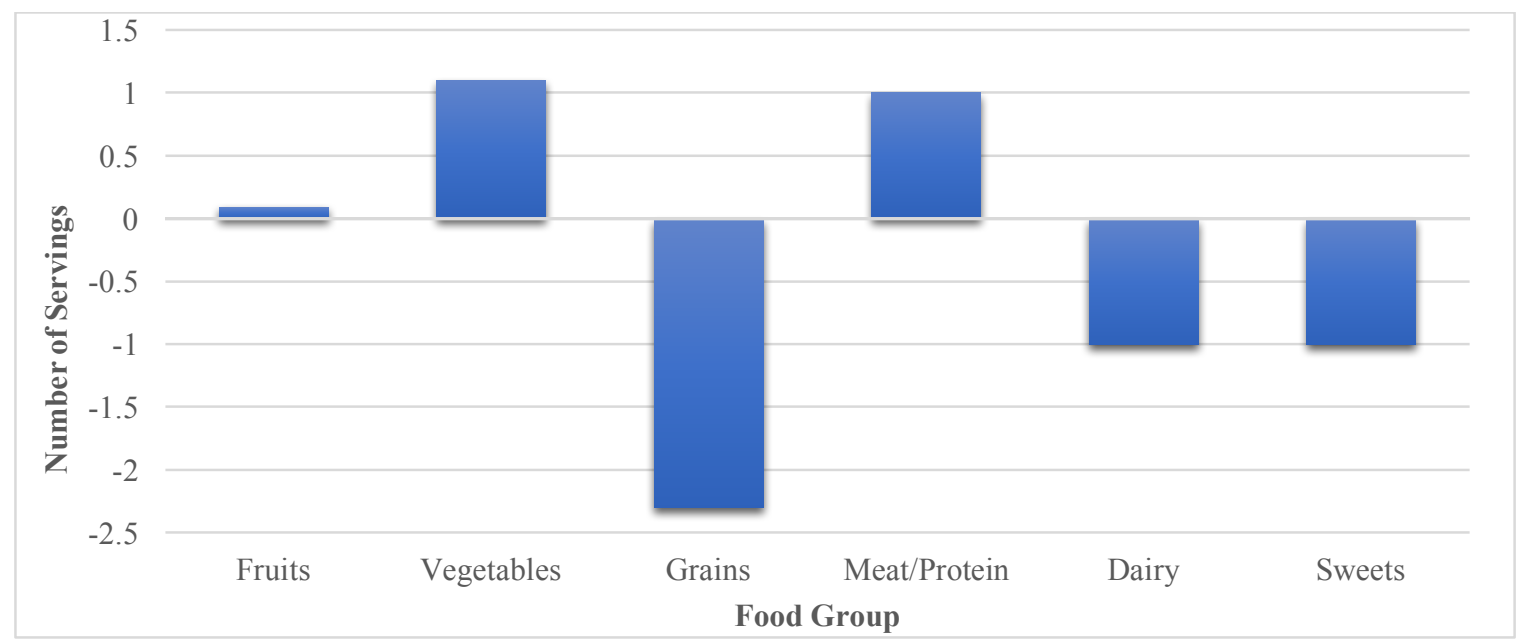

Figure 17. Mean Change in Servings of Food Groups Post-Diet Initiation ( $n=14)$

The largest food group reduction while on the low-FODMAP diet was seen in grains, which decreased by over 2 servings per day. Dairy and sweets also decreased by 1 serving per day. Table 7 reflects the analysis of changes in nutrient and food group consumption from baseline to week two of the low-FODMAP diet.

\begin{tabular}{l|c|c|c}
\multicolumn{1}{|c}{ Variable } & $\begin{array}{c}\text { Mean Intake for } \\
\text { All Participants at } \\
\text { Week 2 }\end{array}$ & $\begin{array}{c}\text { Change from } \\
\text { Baseline to } \\
\text { Week 2 }\end{array}$ & P-value \\
\hline Kcal (mean \pm SD) & 1484 & $-244.5 \pm 667.8$ & .194 \\
\hline CHO, grams (mean \pm SD) & 141 & $-50.2 \pm 77.9$ & $.031^{*}$ \\
\hline $\begin{array}{l}\text { CHO, \% total kcal (mean } \pm \\
\text { SD) }\end{array}$ & 38.7 & $-4.0 \pm 9.3$ & .129 \\
\hline Protein, grams (mean \pm SD) & 69 & $-1.4 \pm 32.8$ & .873 \\
\hline Table 8. Summary of Changes in Nutrient Intake from Baseline to Week Two of the Low- \\
\hline FODMAP Diet
\end{tabular}


Protein, \% total kcal (mean \pm SD)

\begin{tabular}{|l|c|c|c|}
\hline Fat, grams (mean \pm SD) & 65 & $-11.8 \pm 34.9$ & .229 \\
\hline Fat, \% total kcal (mean \pm SD) & 38.2 & $-.79 \pm 6.0$ & .630 \\
\hline $\begin{array}{l}\text { SFA, \% total kcal (mean } \pm \\
\text { SD) }\end{array}$ & 12.1 & $-1.4 \pm 3.7$ & .195 \\
\hline $\begin{array}{l}\text { Added Sugars, \% total kcal } \\
\text { (mean } \pm \text { SD) }\end{array}$ & 8 & $-3.5 \pm 5.1$ & .24 \\
\hline Vitamin D, mcg (mean \pm SD) & 8.1 & $1.4 \pm 3.4$ & .16 \\
\hline Vitamin C, mg (mean \pm SD) & 61.8 & $9.8 \pm 32.9$ & .286 \\
\hline Folate, mcg (mean \pm SD) & 248.1 & $-16.3 \pm 114.6$ & .604 \\
\hline Calcium, mg (mean \pm SD) & 636.9 & $-173.9 \pm 407.3$ & .134 \\
\hline Iron, mg (mean \pm SD) & 11 & $.47 \pm 5.3$ & .745 \\
\hline Fiber, grams (mean \pm SD) & 14.3 & $-.22 \pm 5.2$ & .876 \\
\hline Soluble Fiber, grams (mean \pm & 3.8 & $-1.2 \pm 2.1$ & .051 \\
\hline SD) & & & \\
\hline Insoluble Fiber, grams (mean & 10.2 & $.79 \pm 3.5$ & .422 \\
\hline $\mathbf{\pm}$ SD) & & & \\
\hline Fruit Servings & 1 & $-.04 \pm .81$ & .872 \\
\hline Vegetable Servings & 3.6 & $1.1 \pm 2.2$ & .872 \\
\hline Grain Servings & 3.4 & $-2.3 \pm 3.9$ & $.048^{*}$ \\
\hline Meat Servings & 6.7 & $.99 \pm 2.8$ & .214 \\
\hline Dairy Servings & 1.7 & $-1.0 \pm 1.3$ & $.010^{*}$ \\
\hline Fat Servings & 3 & $-.17 \pm 1.9$ & .743 \\
\hline Dessert Servings & 1.7 & $-.95 \pm 2.2$ & .133 \\
\hline
\end{tabular}
...Continued

There was a statistically significant reduction in consumption of carbohydrates $(\mathrm{p}=0.031)$. However, the reduction in carbohydrates as a percentage of total energy intake was not significant. There were no statistically significant changes in fiber intake from baseline to week two of the low-FODMAP diet. However, there was a trend toward significance for a reduction in soluble fiber intake, but this did not reach significance 
$(p=0.051)$. There was a statistically significant reduction seen for grain $(p=0.048)$ and dairy $(p=0.01)$ intake for participants.

\section{Class Evaluation}

At the end of each class, attendees were asked to complete a class evaluation regardless of participation in the research protocol. The final question of this evaluation form was regarding what type of education session individuals would prefer for dietary advice in the future. Figure 18 displays the distribution of responses for this question.

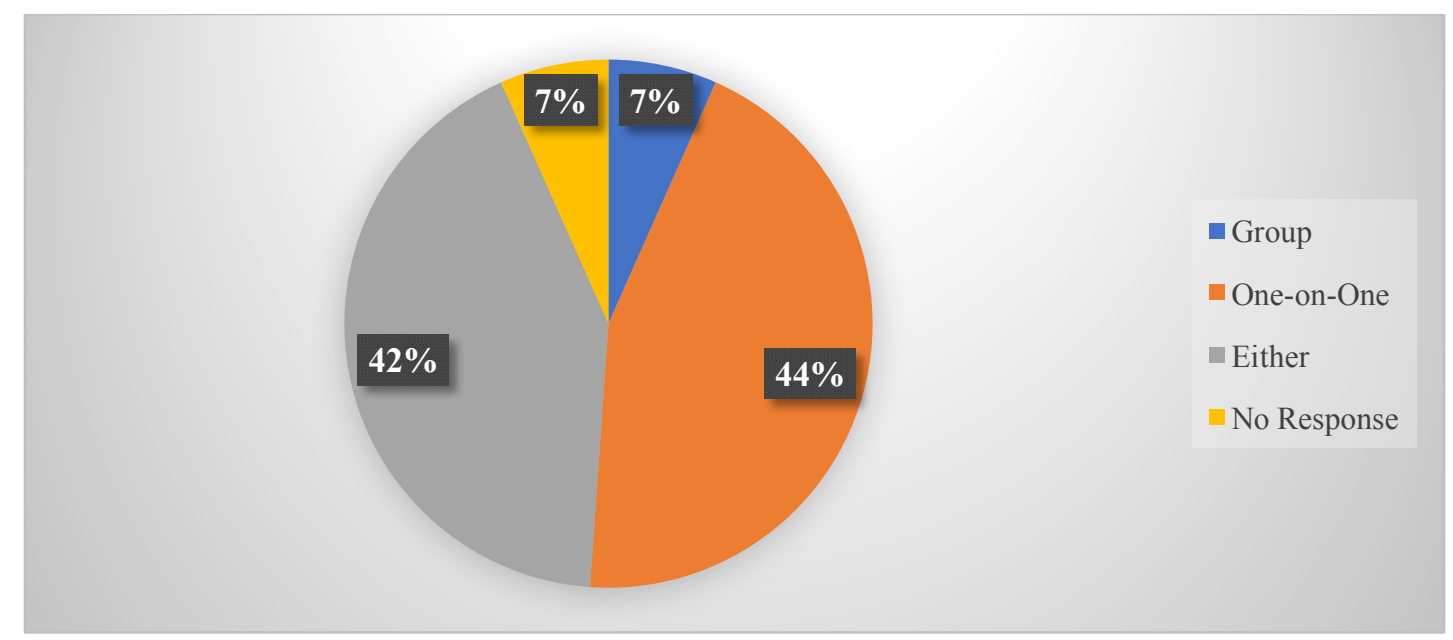

Figure 18. Patients' Preferred Type of Dietary Education Session ( $n=45)$

From the 70 class attendees, we received 45 completed class evaluations. Regarding preference of education session (group versus one-on-one), most class participants would prefer one-on-one education or stated the class format did not matter. Questions regarding session length, class content, class balance, and ability to ask 
questions were also on the class evaluation. For the session length, 42 thought it was "just right." Regarding the content of the class, 42 found the content "just right." For the balance of lecturing and group discussion, 44 found the balance "just right." It appears that most participants had the ability to discuss their concerns and were provided with enough information, with 39 and 30 responding yes to these questions, respectively.

\section{Discussion}

One similarity between participants in this study and the general IBS population is the gender distribution. IBS is more common in females than males. ${ }^{14,17-18}$ This result was seen in the consented IBS patient population and in participants who returned study data, with females representing $77 \%$ and $62.5 \%$ of the groups, respectively. More males who consented to the study participated and returned data when compared to females. Eight males with IBS consented to the study, and of these 8 males, $6(75 \%)$ participated in the study and returned data. For females, there were 27 that enrolled, however only $10(27 \%)$ participated in the study and returned data. There was no statistically significant difference in BMI between participants $(n=16)$ and non-participants $(n=19)$. The age of our individuals in this study differs from the age of the general IBS population. IBS prevalence is known to decrease with increasing age. ${ }^{18}$ A meta-analysis on the global prevalence of IBS found that the odds of IBS are significantly lower in individuals aged 50 years or older. ${ }^{18}$ Our population was older with half $(48.5 \%)$ of the participants being over the age of 50 .

Several studies have reported that IBS patients believe their symptoms are manifested by dietary intake..$^{2-3}$ Of the individuals that consented for this study, $62 \%$ 
claimed to already have been excluding some foods; therefore, these results align with the literature. Dairy, fruits, vegetables, and meats were the most common dietary choices reported as being excluded prior to low-FODMAP diet education. IBS patients have been found to limit certain foods on their own, such as fruits and vegetables, in order to reduce IBS symptoms. ${ }^{2}$ Examples of fruits that were being excluded are apples, cantaloupe, watermelon, and berries. Examples of vegetables that were being excluded are tomatoes, mushrooms, cauliflower, broccoli, Brussel sprouts, cabbage, and peas. It is important to note that researchers in this study are unsure why participants were already excluding these foods and it may not necessarily be due to symptom manifestation.

In this study's IBS patient population, 48.5 and $66 \%$ were using prescription and non-prescription medications, respectively. Eighty percent of participants were utilizing vitamin, mineral, or nutritional supplements. Furthermore, 28.5 and $17 \%$ of IBS patients were utilizing herbal supplements and alternative therapies, respectively. This result is similar to the literature, as around a third of the United States FGID patient population utilizes complementary health approaches. ${ }^{73}$

In this study, a clinical response to the low-FODMAP diet was classified as a reduction $\geq 50$ points in IBS-SSS, which coincides with the IBS-SSS validation article by Francis et al. ${ }^{33}$ Based on this classification, $94 \%$ of study participants ( $n=16$ ) were responders to the diet. ANOVA with repeated measures indicated that there was a statistically significant difference in IBS-SSS from baseline to week two of the dietary intervention. This symptom response in participants indicates that this study's lowFODMAP diet elimination phase recommendation of six weeks is consistent with the literature, which typically recommends an elimination phase between two to eight- 
weeks. ${ }^{10,63,113}$ Usually, in clinical practice, four weeks provides sufficient time for the majority of patients to see improvement in IBS symptoms. ${ }^{9}$ This shorter time for elimination is supported in our study as $87 \%$ of responders $(13 / 15)$ saw a clinical response after two weeks of the diet. The other $13 \%(2 / 15)$ saw a clinical response after six weeks of the diet. Since a statistically significant difference was seen between baseline and week two of the low-FODMAP diet, these results suggest it may only be necessary to follow the low-FODMAP diet elimination phase for two weeks in the majority of IBS patients who are going to clinically respond to the diet. Research has proposed that the low-FODMAP diet may be detrimental to the GI microbiome ${ }^{114,123,129,134}$ and could affect patients' nutritional status, ${ }^{123,125-126,136-137}$ therefore a shorter elimination phase would be best to support patients' overall health.

There are numerous studies that have evaluated the habitual diet for IBS patients and compared them to national/international guidelines. These studies have been conducted all over the world, including Poland, Sweden, Ireland, the Netherlands, and the United Kingdom. ${ }^{2,80-81,147-148}$ Based on this data, it is common to see an increased consumption of fat and a lower consumption of carbohydrates in the IBS patient population. ${ }^{80-81,147}$ At baseline, 9 participants who returned dietary data $(n=14)$ were above the AMDR for fat ( $>35 \%$ of energy intake) and 8 participants were below the AMDR for carbohydrates ( $<45 \%$ of energy intake). ${ }^{144}$ These nutrient distribution patterns continued during the dietary intervention. Furthermore, 13 participants were above the recommended intake ( $>10 \%$ of energy intake) set by the 2015-2020 Dietary Guidelines for Americans for SFA at baseline. ${ }^{145}$ An overconsumption of SFA in the IBS patient population has been expressed in the literature. ${ }^{81}$ This finding regarding the 
overconsumption of SFA remained post-diet initiation as well. It is also common for IBS patients to have a decreased fiber consumption. ${ }^{80}$ An adequate intake of fiber is 14 grams per 1,000 calories consumed. ${ }^{145}$ At baseline, the average fiber intake for men in this study was 16 grams, and the average fiber intake for women was 14 grams. Only 2 participants were meeting the recommendation for fiber at baseline. The underconsumption of fiber remained post-diet intiation as well. Overall, the dietary intake of the participants in this study at baseline was similar with other descriptions for the habitual diet for the IBS patient population.

In the literature regarding IBS patients' habitual diets, IBS patients typically consume the same amount as healthy controls or a higher amount than estimated requirements; ${ }^{147-148}$ however, the findings of this study did not align with this literature. Over half (64\%) of participants were not meeting their estimated energy requirements at baseline. The estimated energy requirements for this population was based on 20-25 $\mathrm{kcal} / \mathrm{kg} / \mathrm{day}$. Before beginning the low-FODMAP diet, participants were consuming a mean of $22.9 \mathrm{kcal} / \mathrm{kg} /$ day. Post-diet initiation, participants' mean energy consumption decreased to $19.5 \mathrm{kcal} / \mathrm{kg} / \mathrm{day}$. Six participants saw a reduction in energy intake after initiating the low-FODMAP diet, and the mean energy reduction was $844 \mathrm{kcals}$ for these six participants. Eight participants were below their estimated energy requirements postdiet initiation, 6 of which were already consuming below their estimated energy requirements at baseline. Two participants who were consuming above their estimated energy requirements at baseline reduced their energy intake below estimated requirements after beginning the low-FODMAP diet. Five participants were consuming less than $1200 \mathrm{kcal} / \mathrm{day}$ while following the low-FODMAP diet, which makes it highly 
unlikely that they are able to meet all of their nutritional needs. While 2 of these 5 participants were already consuming less than $1200 \mathrm{kcal} /$ day at baesline, this result is still concerning. A reduction in energy intake has been seen in low-FODMAP diet studies previously. ${ }^{125-126,131,136}$ While a reduction in energy intake was seen in this study's participants, several participants already reported an energy intake below estimated requirements prior to diet initiation. The reduction in energy intake post-diet initiation was not statistically significant, which may be due to the small sample size in this study and the large variance in the data $(-244.5 \pm 667.8 \mathrm{kcal})$. However, $36 \%(5 / 14)$ of participants were consuming a very low $(<1200 \mathrm{kcal} /$ day $)$ number of calories while following the low-FODMAP diet. Therefore, adequate energy intake during the lowFODMAP diet should remain a concern in future studies.

Carbohydrate intake is of specific concern on the low-FODMAP diet, because the diet involves the elimination of many carbohydrate containing dietary choices. In this study, 11 participants were below the recommended range of carbohydrates post diet initiation $\left(<45 \%\right.$ of energy intake $\left.{ }^{144}\right)$; however, 7 of these 11 participants below the recommended range were already below the AMDR for carbohydrates at baseline. ${ }^{144}$ Moreover, only 3 participants were within the AMDR for carbohydrates (45-65\% of energy intake ${ }^{144}$ ) while following the low-FODMAP diet. The mean grams of carbohydrates consumed was reduced by a 50 grams for all participants, and the percentage of calories from carbohydrates was reduced by a mean of $4 \%$ after initiating the low-FODMAP diet. There was a statistically significant reduction in grams of carbohydrates consumed $(\mathrm{p}=0.031)$ by participants, but the reduction in carbohydrates as a percentage of total energy was not statistically significant. This finding of a reduction in 
carbohydrate consumption while following the low-FODMAP diet is similar to those of other studies, as several randomized controlled trials have found a reduced intake of carbohydrates during the low-FODMAP diet. ${ }^{123,125-126,136}$

Dietary fat intake as a percentage of total calories was reduced for 8 participants after initiation of the low-FODMAP diet; however, it continued to be above the AMDR for fat $\left(>35 \%\right.$ of calories $\left.^{144}\right)$ for 9 participants. Seven of these 9 participants were also above the recommended range pre diet initiation. No participants were below the AMDR for fat while following the low-FODMAP diet. SFA intake was specifically evaluated in this study. The Dietary Guidelines Advisory Committee (DGAC) has found that saturated fat is overconsumed by Americans according to the Tolerance Upper Intake Level (UL) set by the Institute of Medicine (IOM). ${ }^{149}$ Thirteen participants were above the SFA recommendation $\left(>10 \%\right.$ of total energy $\left.{ }^{145}\right)$ at baseline, and 8 remained above the recommendation after the initiation of the low-FODMAP diet. Many dietary options high in SFA, like processed foods, are also high in certain FODMAPs and could have led to the reduction in SFA intake on the low-FODMAP diet. Dairy products are another contributor of SFA in the diet, and many of these are eliminated on the low-FODMAP diet. Current literature regarding the low-FODMAP diet states that fat intake was unchanged after following the low-FODMAP diet, and our data was accordance with this. ${ }^{123,125-126}$ The change in fat and SFA after diet initiation was not statistically significant, and fat intake was not below the AMDR for any participants.

Protein intake as a percentage of total calories decreased for 5 participants after starting the low-FODMAP diet. A majority of participants (93\%) still fell within the AMDR for protein $\left(10-25 \%\right.$ of total energy $\left.{ }^{144}\right)$ while following the diet, with only one 
participant below the AMDR. The reduction in grams of protein consumed was not statistically significant, and protein as a percentage of total calories after initiation of the low-FODMAP diet actually increased, although this was not statistically significant either. This study's results are consistent with current literature, which states that the total protein intake after following a low-FODMAP diet is unchanged. ${ }^{123,125-126}$

This study evaluated several micronutrients, which include folate, vitamin C, vitamin $\mathrm{D}$, calcium, and iron. Few studies have evaluated micronutrient intake while following the low-FODMAP diet. ${ }^{123,131,136}$ These specific micronutrients were chosen for several reasons. According to the DGAC, some of these nutrients (vitamin C, vitamin D, folate, and calcium) were identified as shortfall nutrients because they have been found to be under-consumed in the US based on the IOM's RDA or Adequate Intake (AI) levels. ${ }^{149}$ Calcium and vitamin D have also been categorized as nutrients of public health concern because their under-consumption has been linked to adverse health outcomes. ${ }^{149}$ Iron is also categorized as a shortfall nutrient and a nutrient of public health concern for adolescent and adult females due to an increased risk of iron deficiency in females. ${ }^{149}$ Many IBS patients are females, ${ }^{14,17-18}$ and $77 \%$ of the IBS population enrolled in this study were females. Furthermore, Fulgoni et al. ${ }^{150}$ found through an analysis of usual intake in the United States that many Americans had usual intakes below the EAR for vitamin C, D, and calcium (25, 70, and 38\%, respectively). Enrichment and/or fortification contributes to intake of vitamin D, folate, and iron. ${ }^{150}$ Grain products are enriched and/or fortified with folate and iron, and dairy products are fortified with vitamin D; moreover, the low-FODMAP diet restricts some of these products. ${ }^{150}$ 
Vitamin C, vitamin D, folate, calcium, and iron intake all decreased after the initiation of the low-FODMAP diet by $50,36,57,71$, and $29 \%$, respectively. None of the changes in micronutrients were statistically significant. Most participants were already below the RDA for these micronutrients at baseline, prior to beginning the lowFODMAP diet. Specifically, 12 participants were not meeting the RDA for vitamin C (90 $\mathrm{mg}$ for men, $75 \mathrm{mg}$ for women), 12 were not meeting the RDA for vitamin D (15 mcg for men and women), 13 were not meeting the RDA for folate (400 mcg for men and women), 10 were not meeting the RDA for calcium (1000 mg for men and women), and 11 were not meeting the RDA for iron ( $8 \mathrm{mg}$ for men, $18 \mathrm{mg}$ for women) at baseline. ${ }^{145}$ Furthermore, recommending a multivitamin to patients with IBS may be beneficial.

Of the micronutrients evaluated in this study, calcium had the largest reduction post-diet initiation, as it decreased for 10 participants. While there was a reduction in calcium intake for participants in this study, many of these participants were already below the RDA for calcium pre-diet initiation, and there was not a statistically significant change in calcium intake after initiating the low-FODMAP diet $(p=0.134)$. In the study by Böhn et al., ${ }^{147}$ IBS patients were consuming significantly lower calcium through their usual dietary intake when compared to controls. Furthermore, Prescha et al. ${ }^{81}$ found that only $12 \%$ of females and $14 \%$ of males in their study were meeting the Polish RDA for calcium from their regular dietary intake. Our study results agree with those of Böhn et al. ${ }^{147}$ and Prescha et al., ${ }^{81}$ as 10 participants in our study were not meeting the RDA (1000 mg for men and women ${ }^{145}$ ) for calcium pre-diet initiation. Three more participants' intakes fell below the RDA for calcium post-diet initiation, while 1 participant did increase calcium intake to above the RDA post-diet initiation. Staudacher et al. ${ }^{123}$ found a 
lower calcium intake for subjects following the low-FODMAP diet when compared to habitual diet, but a reduction was not seen in the study by Harvie et al. ${ }^{131}$

Staudacher et al. ${ }^{137}$ found a significant reduction in iron intake after following a low-FODMAP diet, but our study results for iron were not statistically significant. Many participants' intake of iron actually increased after following the low-FODMAP diet in this study, which could possibly be due to an increased intake of meat. Our study was similar to that of Staudacher et al. ${ }^{137}$ in that the proportion of participants following a low-FODMAP diet who were not meeting dietary reference values for iron did not differ from baseline to follow-up. Overall, micronutrient intake should continue to be monitored in future low-FODMAP diet studies for trends and further insight.

Fiber was also classifed as a shortfall nutrient and nutrient of public health concern by the DGAC. ${ }^{149}$ Fiber intake has the potential to be reduced on the lowFODMAP diet due to the diet's restriction of fructans, galacto-oligosaccharides, and various fruits and vegetables. However, several RCTs have found that fiber intake on a low-FODMAP diet does not differ when compared to controls. ${ }^{123,125-126}$ A reduction in fiber intake has been noted while following the low-FODMAP diet, ${ }^{131}$ but this was resolved after reintroduction of FODMAPs containing fiber. Seven of this study's participants had a reduction in total fiber intake after starting the low-FODMAP diet. Only 1 participant was meeting the recommendation for fiber while following the lowFODMAP diet, and only 2 participants were meeting the recommendation for fiber at baseline. While this study did not find a statistically significant reduction in total dietary fiber intake after initiating the low-FODMAP diet, the low intake of fiber at baseline and week two of the low-FODMAP diet is concerning and possibilities to increase fiber 
intake should be evaluated. For soluble and insoluble fiber, $79 \%$ and $43 \%$ had a reduction, respectively. The reduction in soluble fiber trended toward statistical significance $(\mathrm{p}=0.051)$. Beans and nuts are common high sources of soluble fiber, and some of these are restricted on the low-FODMAP diet, which could explain the greater reduction in soluble fiber. The mean change in insoluble fiber for participants increased by 0.79 grams, but this change was not statistically significant.

While following the low-FODMAP diet, changes in the consumption of food groups is expected due to its restrictive nature. Dairy products, grains, fruits, and vegetables are specifically expected to be affected on the low-FODMAP diet since many of them are restricted. However, some of these results did not occur in our study. It was found that vegetable intake increased by over 1 serving per day, and fruit intake stayed close to the same as baseline. The low-FODMAP education class that patients attended before participating in this study focused on proper replacement of foods, such as fruits and vegetables, to still consume a nutritionally adequate diet. Proper education could be the reason why the intake of fruits and vegetables did not decrease during the lowFODMAP diet. This emphasizes the importance of a low-FODMAP diet education to be led by a trained dietitian. There was a statistically significant reduction in grains $(\mathrm{p}=0.48)$ and dairy $(\mathrm{p}=0.010)$ while following the low-FODMAP diet. Many commonly consumed grains, such as wheat, rye, and barely, are required to be avoided during the lowFODMAP diet elimination phase. A reduction in grains is concerning, because grains such as wheat, rye, and barley, are important sources of carbohydrates, fiber, B vitamins, and iron. ${ }^{151}$ Many dairy products, including milk, evaporated milk, and yogurt, are also required to be avoided during the elimination phase. A reduction in dairy is concerning 
because dairy products provide calcium, fat-soluble vitamins, and are fortified with vitamin D. ${ }^{150,151}$ The significant reductions in these groups supports the importance of confirming that patients are reintroducing FODMAPs properly and as soon as they can to promote a well-rounded intake and avoid inadequacies. This further emphasizes the important role a trained RDN plays in low-FODMAP diet education. The intake of sweets also reduced by about one serving, but this was not statistically significant. It is not surprising that the intake of sweets reduced, since the diet restricts grains, sugar alcohols, and high fructose corn syrup.

Compliance to the diet varied, but overall the number of reported events of noncompliance were low. The average intake of lactose, fructose, and sugar alcohols all decreased from baseline to week 2 of the low-FODMAP diet, which supports participant compliance to the diet. Incidences of noncompliance ranged from 1-24 days, with a mean of 2 days for all participants. The most common high-FODMAP foods consumed while on the diet were dairy products, wheat, garlic, and onion. Dairy products were consumed by 6 participants one or more times. Specific dairy products that were consumed are milk, yogurt, cottage cheese, and ice cream. Wheat was consumed by 5 participants one or more times. Garlic, onion, and/or garlic and onion salt/powders are in many processed food products, such as sauces and condiments. Garlic and/or garlic salt/powders were consumed by 6 participants one or more times. Onion and/or onion salt/powders were consumed by 5 participants one or more times. While compliance to the diet was acceptable for some participants, a poor compliance to the diet could indicate the potential for a placebo effect related to IBS-SSS scores. Two studies have reported evaluating dietary compliance, but this was done by questioning study participants about 
their compliance to the diet ${ }^{10,132}$ rather than with a specific instrument such as this study's high-FODMAP checklist. The high frequency of dairy, wheat, garlic and onion intake and poor compliance to the diet reported by some participants in this study highlights the need to assess compliance in future interventions to determine if a reduction in IBS-SSS is a result of a reduction in FODMAP concentration or a placebo effect.

Of the 35 IBS patients who consented to the study, 14 (40\%) participants returned complete data and $2(6 \%)$ returned incomplete data. Nineteen (54\%) participants dropped out of the study. The low-FODMAP diet is very strict and cumbersome to follow. The literature has reported that common barriers in compliance to the diet are cost of alternative dietary products, low palatability of some speciality food products, and limited options for eating outside of home. ${ }^{9}$ The most typical response when asking this study's participants why they dropped out of the study was related to the diet being too strict or not fitting into their social/personal lives at the time. Two participants also reported that the diet was potentially making them feel ill while they were following it. One participant reported no bowel movement for two weeks, while the other participant reported headaches, fatigue, and increased appetitite. In the future, RDNs and healthcare providers should address potential challenges of the diet and discuss potential strategies to overcome these barriers. A shorter elimination phase would also be beneficial to promote compliance. This study found that an elimination phase of two weeks was statistically significant in promoting a clinical response to the diet. If patients are required to follow the diet for a shorter period of time, this will likely increase compliance and improve the dropout rate in future studies. 
While this diet has shown success in relieving IBS symptoms, the lack of compliance and high dropout rate shows that it may not be the best method to recommend for some patients. Since general "healthy eating advice" has been shown to be as effective as the low-FODMAP diet for some symptom outcomes, ${ }^{125-126}$ it is recommended that it be attempted prior to the low-FODMAP diet as these changes may be more receptive to patients. ${ }^{9}$ Moreover, the meta-analysis by Varju et al. ${ }^{121}$ found that there was a significant reduction in IBS-SSS score for both standard dietary advice groups and low-FODMAP diet groups. In the meta-analysis by Altobello et al., ${ }^{120}$ it was found through RCTs and cohort studies that patients receiving the low-FODMAP diet had statistically significant reductions in pain and bloating compared to a traditional diet (in RCTs) or to baseline symptoms (in cohort studies). For the RCTs comparing the lowFODMAP diet to traditional diets there were not significant differences in stool consistency or stool frequency between the two diets. ${ }^{120}$ Due to the nature of this diet and the lack of compliance found through our study, it may be beneficial to only recommend the low-FODMAP diet to patients who are experiencing extreme pain and bloating. If a patients primary symptom concerns are stool consistency or stool frequency, the lowFODMAP diet may not be the best dietary approach to use initially. Clinical effectiveness for the low-FODMAP diet has only been seen with education led by a RDN-led education. ${ }^{9}$ When possible, it is important that dietary counseling is tailored to the patient and the RDN assessses what dietary approach best fits their IBS symptoms, lifestyle factors, and ability to understand and remain compliant to the more demanding dietary regimen. 
Most participants who completed the class evaluation were satisfied with the structure of the class. Participants believed they had the ability to talk about everything they wanted and were provided with enough information through the class education session. The literature has shown there is no significant difference in patient response to the low-FODMAP diet when provided with a group education session versus a one-onone session. ${ }^{143}$ For those who felt as if they still left with questions or were not provided with enough information, there may be a relationship between educational satisfaction and compliance to the diet. However, it is impossible to link non-compliance to satisfaction with the class as the evaluations were anonymous. Even though most patients were content with the group education session, close to half of participants reported they would rather receive diet advice in a one-on-one setting. This could be due to complexity of the diet, as some participants did not find the group session long enough and felt as if the information was too complex. This also could be related to personal preference, as some participants noted in their comments that they feel they need an individual appointment to acknowledge their specific IBS-related concerns. There is a limited supply of dietitians with appropriate expertise to counsel on the low-FODMAP diet, ${ }^{9}$ therefore a group education session is an appropriate alternative approach for dietitians with appropriate training to provide this education and counseling. Regardless of session type, is important that low-FODMAP dietary education is dietitian-led. An improper delivery method of the low-FODMAP diet could lead to incomplete FODMAP restriction or an over-restrictive dietary intake, leading to lack of effectiveness or nutritional inadequacies, respectively. ${ }^{9}$ 


\section{Limitations}

A major limitation of this study is its sample size. A smaller sample size can affect a study's power and cause an inability to perform statistical analysis. While this study did find statistical significance for some variables, it is possible that there may be a significant difference in other variables that was not found due to a low power. However, other FODMAP studies have similar sample sizes in their cohort or intervention group. $^{107,123,129}$

Randomized controlled trials are the gold standard for clinical studies. Our study lacks a control group to support that the results of the intervention were truly due to the intervention performed. Our study also lacks blinding, however, since the intervention in this study was a change in dietary pattern, the opportunity for participant blinding was not possible. There may be a sampling bias present in this study since participants were recruited from a clinic that specializes in gastroenterology, while most individuals with IBS are diagnosed and managed at a primary care clinic. The US community survey study by Hungin et al. ${ }^{13}$ found that $79 \%$ of medically diagnosed IBS sufferers and $85 \%$ of non-medically diagnosed IBS sufferers consulted a primary care physician for their symptoms. However, only 64 and $26 \%$ of medically and non-medically diagnosed IBS

sufferers consulted a gastroenterologist, respectively. ${ }^{13}$ Since the participants in our study came from a gastroenterology clinic, this could indicate that they suffer more severe symptoms and were more likely to attempt such a challenging and restrictive diet. Over half $(54 \%)$ of the consented individuals and participants who returned data $(56 \%)$ were categorized as having severe IBS, at baseline, according to their IBS-SSS scores. 
Other major limitations in this study are using patient-reported data and being forced to rely on participants to return complete data. Efforts were made to minimize patient burden through providing envelopes, stamps, and reminder calls or texts; however, some participants did not return any documents or did not include all documents in their returned data. With patient reported data, there is a risk of bias regarding the data that is returned. It is possible that the patients who returned data did so because they were experiencing success on the low-FODMAP diet and therefore continued following it. Participants who did not return data may have failed to see results on the diet and did not feel required to return their data.

Furthermore, in a dietary intervention study, there is also the possibility for reactivity, which is a change in behavior due to participants to being monitored. Reactivity could have affected participant's three-day diet record documentation. There is a potential for a placebo effect in this intervention where participants may have reported an improvement in IBS-SSS because of participation in the study rather than an actual response to the diet. For all study instrumentation returned by participants, there is the possibility of human error during the documentation of IBS symptoms and dietary intake.

Researchers in this study are unable to fully determine compliance to the lowFODMAP diet. While researchers did provide daily high-FODMAP checklists to fill out, it is impossible to know if participants had full compliance since researchers did not require daily diet records. Given the potential distressing effects of asking participants to complete food records and challenges with accountability, ${ }^{139}$ reliance on food checklists was selected over daily food records. Diet records require motivated and literate individuals to complete, and participants may feel that having to document dietary intake 
may disrupt their lives or makes them feel socially awkward. ${ }^{139,152}$ Some participants may also feel discouraged because journaling is a constant reminder of their dietary choices. ${ }^{139}$ Moreover, the knowledge that foods and amounts will be recorded and the burden of doing so may alter participant's dietary behaviors. ${ }^{142,152}$ Longer reporting periods are typically difficult for patient compliance and reported intake may decrease due to patient fatigue, and the validity of data collected decreases in the later days of longer reporting periods. ${ }^{152}$ Our study measured dietary intake using diet records at baseline and after twoweeks of following the low-FODMAP diet. Therefore, this study is also limited to the short-term effects of the low-FODMAP diet on nutrient intake, but it cannot attest to the long-term effects of following this diet.

\section{Recommendations for Future Studies}

Throughout this discussion, nutrient intake and dietary compliance has been discussed extensively. Prior to beginning the low-FODMAP diet, it is important that RDNs and healthcare providers are completing an accurate assessment of current nutrient and FODMAP intake. The Monash University Comprehensive Nutrition Assessment Questionnaire (CNAQ), ${ }^{153}$ which was developed and validated by Barrett and Gibson ${ }^{154}$ with a group of Australian adults, is a FFQ that can assess a wide range of nutrients, including FODMAPs. This tool is useful for researchers studying gastrointestinal disorders, especially IBS. Utilizing the CNAQ in FODMAP research at baseline, during, post-dietary intervention would be a valuable addition to other dietary intake measures.

It has been highlighted in the literature that IBS affects individuals'

HRQOL. ${ }^{13,20,25}$ Several low-FODMAP studies have evaluated HRQOL and discovered an 
improvement after following the low-FODMAP diet. ${ }^{118-119}$ The IBS-QOL questionnaire, developed Patrick et al., ${ }^{155}$ meets established psychometric criteria for validity and reliability. Since the IBS-QOL was constructed specifically for IBS patients, it would be a useful instrument to utilize in future studies. While there are other tools that can measure quality of life in the IBS patient population, the IBS-QOL has been established as the preferred tool for changes in IBS-specific quality of life. ${ }^{156}$

\section{Conclusion}

The findings of this study suggest a low-FODMAP diet elimination phase of two weeks appears optimal for achieving symptom relief. Due to the restrictive nature of the low-FODMAP diet, an inadequate intake of nutrients and deficiencies in micronutrients are possible, although there is limited research in this area. There was a significant reduction in grain and dairy intake, which could affect the overall consumption of both macro- and micronutrients. There was also a lower carbohydrate consumption after beginning the low-FODMAP diet for participants in this study. Moreover, the lowFODMAP diet may lead to a nutritionally inadequate dietary pattern and must be monitored in future studies. A shorter total elimination period would help prevent nutritional inadequacies and could improve patient compliance. The poor compliance reported in this study highlights the need to assess compliance in future interventions to determine if a reduction in IBS-SSS is a result of FODMAP concentration or placebo effect. This study proposes that a proper dietitian led education session can help achieve adequate nutritional intake by educating patients on proper substitutions of FODMAP containing foods, and a group education session is suitable for this dietary intervention. 
More rigourous studies with a larger sample size are necessary to evaluate nutritional adequacy and compliance with the low-FODMAP diet. 


\title{
Chapter 5: Journal Article
}

\section{Evaluation of Nutritional Adequacy and Symptom Improvement During Implementation of the Low-FODMAP Diet in Individuals with Irritable Bowel Syndrome}

Keywords: IBS, low FODMAP, symptom relief, IBS-SSS, diet adequacy, dietary changes, compliance

\begin{abstract}
Background: Irritable bowel syndrome (IBS) is a common functional gastrointestinal disorder (FGID) that affects $10-20 \%$ of adults and adolescents worldwide. A connection has been identified between the consumption of some foods and an exacerbation of IBS symptoms. One nutrition therapy option for IBS is the low fermentable oligosaccharides, disaccharides, monosaccharides, and polyols (FODMAP) diet. A lack of data describing the mechanism of action of this dietary pattern has led to insufficient evidence to establish standard clinical guidelines regarding the use of the low-FODMAP diet in IBS. Objective: This study aims to define the duration of the low-FODMAP diet elimination phase for optimal symptom relief and the nutritional adequacy of the low-FODMAP diet. Methods: Subjects attended a low-FODMAP diet group education class and were instructed to follow the low-FODMAP diet for six weeks. Subjects completed an IBS-
\end{abstract}


Symptom Severity Scale questionnaire (IBS-SSS) at baseline and weeks 2, 4, and 6.

Subjects completed a three-day diet record at baseline and week 2. Dietary compliance was assessed through daily high-FODMAP food checklists.

Results: A total of 35 participants enrolled in this study. Complete data was available for $14(40 \%)$ participants. Incomplete data was available for $2(6 \%)$ participants, and 19 (54\%) dropped out of the study. 43\% (15/35) participants were responders to the diet (a reduction $\geq 50$ points in IBS-SSS), and 87\% (13/15) of responders saw a clinical response after two weeks of the diet. There was a significant reduction in symptoms seen between baseline and week two $(\mathrm{p}<0.001)$. Post initiation of the low-FODMAP diet in those participants with complete data, there was a statistically significant reduction in grams of carbohydrates consumed $(\mathrm{p}=0.031)$. Servings from grains and servings from dairy were also significantly reduced $(\mathrm{p}=0.048, \mathrm{p}=0.010)$. Calcium, iron, folate, vitamin $\mathrm{C}$, and vitamin D intake were below the recommended dietary allowance (RDA) for $>75 \%$ of participants. $71 \%$ of participants reported non-compliance.

Conclusion: Our findings support that a low-FODMAP diet elimination phase of two weeks is optimal for achieving symptom relief. The low-FODMAP diet leads to a nutritionally inadequate dietary pattern and must be monitored in future studies. The high dropout rate and poor dietary compliance reported in this study highlight the need to assess dietary compliance in future interventions to determine if a reduction in IBS-SSS is a result of dietary FODMAP concentration or placebo effect. 


\section{Introduction}

Irritable bowel syndrome (IBS) is a functional gastrointestinal disorder (FGID) characterized by lower abdominal pain or discomfort associated with a change in stool frequency or consistency. Rome III Criteria classifies IBS as recurrent abdominal pain or discomfort lasting three days per month for at least three months that is accompanied by two of the following: improvement with defecation, onset related to a change in stool frequency, or onset related to a change in the appearance of stool. ${ }^{5}$ IBS affects $10-20 \%$ of adults and adolescents worldwide and drastically impacts an individual's work productivity and health related quality of life (HRQOL). ${ }^{1,13,20,25}$ In the United States, the mean direct cost of IBS per patient is $\$ 619$ annually, and total annual direct costs related to IBS are $\$ 1.35$ billion. ${ }^{15}$

While IBS is a complex, multifactorial disorder, a connection between exacerbation of symptoms and the ingestion of food has been determined through the literature. ${ }^{2-3}$ Previously, a concrete dietary approach for the management of IBS symptoms had not been established; therefore, medical management relied heavily on pharmaceuticals for symptom relief. Over the past decade, the low fermentable oligosaccharides, disaccharides, monosaccharides, and polyols (FODMAP) diet has gained attention for being an effective management approach for the symptoms of IBS through both controlled and uncontrolled clinical trials. ${ }^{6}$ The low-FODMAP diet removes all short-chain, poorly absorbed carbohydrates that cause an osmotic effect on the bowel and excess gas production due to fermentation by intestinal bacteria. This dietary approach is split into three distinct stages: FODMAP elimination, FODMAP 
reintroduction, and FODMAP personalization. ${ }^{9}$ While there is no standardized period of time for the elimination phase, it is typically for a two to eight-week period. ${ }^{10,63,113}$

The low-FODMAP diet is highly restrictive of fruit, vegetables, dairy, and grains, particularly during the elimination phase. Such a restrictive diet for an extended period raises concern for inadequate nutrient intake and micronutrient deficiencies. This study aims to define the necessary duration of the low-FODMAP diet elimination phase for optimal symptom relief and the nutritional adequacy of the low-FODMAP diet's elimination phase.

\section{Methods}

The Ohio State University Institutional Review Board approved this study on November 2, 2016 (IRB\# 2016H0320). Individuals diagnosed with IBS based on the Rome III Criteria who were referred to The Ohio State University Medical Center's Division of Gastroenterology, Hepatology, and Nutrition for a consultation with a RDN were scheduled to attend a group education class for the low FODMAP diet. A total of 15 males and 55 females attended the class and $50 \%$ were consented to participate in the study. Information learned through the lecture was followed by practical application and problem solving activities via discussions and the use of visual examples. Educational materials created by Kate Scarlata, RDN, LD were used in the class and in participants' binders to take home as a resource. Participants received a notebook of educational materials and copies of study instruments. Prior to leaving, participants completed the consent process and filled out the baseline IBS-SSS and baseline health/demographic questionnaires. Participants were instructed to follow the low-FODMAP diet elimination 
phase for six weeks. Participants completed a three-day diet record at baseline and week two of the low-FODMAP diet. Participants completed an IBS-SSS questionnaire at baseline, and weeks two, four, and six. A clinical response to the low-FODMAP diet was classified as a reduction $\geq 50$ points in IBS-SSS, which coincides with the IBS-SSS validation article by Francis et al. ${ }^{33}$ If a FODMAP-containing food was consumed, participants were instructed to document the food item and amount on daily highFODMAP checklists provided. A summary of study interventions is provided in figure 1. Participants were contacted every two weeks, via phone, text, or email communication, to check status and answer any questions.

\section{Inclusion/Exclusion Criteria}

Inclusion criteria included: age $>18$ years, ability to read English, and a diagnosis of IBS and referral for nutrition consultation/class attendance from provider. Exclusion criteria included inability to provide informed consent for the study (including prisoners) and pregnancy.

\section{Data Analysis}

Data on dietary intake collected using the three-day diet records was analyzed using the Nutrition Data System for Research software (NDSR), version 2016, developed by the Nutrition Coordinating Center (NCC) at the University of Minnesota in Minneapolis, MN. Trained professionals at The Ohio State University in the Clinical Research Center's (CRC) Nutrition Research Laboratory entered dietary intake data. Quality assurance procedures per NDSR protocol were executed, including review of the data by three different individuals for consistency in interpretation and verifying 
questionable data with the study participant. To summarize the dietary intake data, means and standard deviations were reported for continuous variables. Frequencies and percentages were used to summarize categorical data. Paired sample t-tests were used to assess changes in nutritional intake from baseline to week two based on data from the three-day diet records. An ANOVA with repeated measures was used to compare differences in continuous measures of symptoms measured by the IBS-SSS. A Bonferroni correction was applied for multiple comparisons. Independent samples t-tests were used to assess differences between participants and non-participants. Results are presented as point estimates with $95 \%$ confidence intervals and significance was defined as a twosided p-value of less than 0.05 .

\section{Results}

\section{Study Enrollment}

Seventy patients with IBS attended the RDN-led low-FODMAP education classes. Thirty-five participants (50\%) consented to the study. Of the study participants, $14(40 \%)$ returned complete data, and $2(6 \%)$ returned incomplete data. The flow of class attendance and study enrollment is displayed in figure 2 .

Demographic, Lifestyle, and Baseline Health Characteristics

Data reported in this section reflects information from all participants who enrolled and consented to the study ( $\mathrm{n}=35)$. Twenty-seven IBS patients were females and 8 were males. The mean age of the population was $47.7 \pm 16.3$ years. The mean BMI was $28.7 \pm 8 \mathrm{~kg} / \mathrm{m}^{2}$, which is categorized as overweight. There was no statistically significant difference between participants ( $\mathrm{n}=16)$ versus non-participants $(\mathrm{n}=19)$ for age or BMI. 
Twenty-eight IBS patients reported participating in some form of physical activity. Three IBS patients reported smoking and 15 reported a mild intake of alcohol (less than 7 drinks/week or 14 drinks/month). Six participants ( $\mathrm{n}=16)$ were IBS-D, 3 were IBS-C, 3 were IBS-M, and 4 were IBS-U based on their Bristol Stool Form Scale results at baseline. A summary of this information is presented in table 1.

\section{Symptom Changes}

Data reported in this section reflects the 16 participants who returned IBS-SSS data. Fifteen (94\%) participants reported a clinical response to the low-FODMAP diet based on their IBS-SSS scores over the 6-week study period. A statistically significant reduction in IBS-SSS scores were found between baseline and week $2(\mathrm{p}<0.001)$, baseline and week $4(p=0.002)$, and baseline and week $6(p<0.001)$. There were no other statistically significant differences between weeks. Table 2 displays the statistical analysis results. For the participants who saw a clinical response to the low-FODMAP $\operatorname{diet}(\mathrm{n}=15), 13$ participants responded after two weeks. The other 2 responders saw a clinical response after six weeks of the diet.

\section{Changes in Total Energy Intake}

Data reported on nutritional adequacy of the low-FODMAP diet reflects the 14 participants who returned three-day diet records from baseline and week two of the intervention. Six (43\%) participants had a reduction in total calories per day when following the low-FODMAP diet, and 50\% (3/6) of these participants had a reduction $>50 \%$ in total calories per day. There were 5 participants who consumed under 1200 $\mathrm{kcal} / \mathrm{day}$ after initiating the low-FODMAP diet, and 2 of these 5 participants were already consuming under $1200 \mathrm{kcal} /$ day prior to initiating the diet. 


\section{Changes in Macronutrients}

At week two of the low-FODMAP diet, there was a mean reduction in carbohydrate and fat consumption for participants, and a mean increase in protein consumption. A statistically significant reduction in grams of carbohydrates consumed was seen at week two of the low-FODMAP diet $(\mathrm{p}=0.031)$. There were no other significant changes from baseline to week two for any macronutrients. A summary of these changes is presented in table 3 . At two weeks, carbohydrate intake was below the AMDR $\left(<45 \%\right.$ of energy intake $\left.{ }^{144}\right)$ for 11 participants, and fat intake was above the $\operatorname{AMDR}\left(>35 \%\right.$ of energy intake $\left.{ }^{144}\right)$ for 9 participants. Table 4 summarizes the distribution of macronutrients for participants post-diet initiation.

\section{Changes in Micronutrients}

Vitamin D intake decreased for 5 participants post initiation of the low-FODMAP diet. Twelve participants were not meeting the RDA for vitamin D (15 mcg for men and women $^{145}$ ) pre-diet initiation, and 1 additional participant's intake fell below the RDA post-diet initiation. Vitamin $\mathrm{C}$ intake decreased for 7 participants post diet initiation. Twelve participants were not meeting the RDA for vitamin C $(90 \mathrm{mg}$ for men and $75 \mathrm{mg}$ for women ${ }^{145}$ ) pre-diet initiation. Post-diet initiation, 1 of these participants increased vitamin $\mathrm{C}$ intake above the RDA; however, the other 11 participants remained below the RDA for vitamin C. Folate intake decreased for 8 participants post-diet initiation. Thirteen participants were not meeting the RDA for folate $(400 \mathrm{mcg}$ for men and women $^{145}$ ) pre-diet initiation. Post diet initiation, 1 of these participants increased folate intake above the RDA; however, the other 12 participants remained below the RDA for 
folate. Calcium intake decreased for 10 participants post initiation of the low-FODMAP diet. Ten participants were not meeting the RDA (1000 mg for men and women $\left.{ }^{145}\right)$ for calcium pre-diet initiation, and 3 more participants' intakes fell below the RDA for calcium post-diet initiation. One participant that was previously below the RDA for calcium pre-diet initiation did increase calcium intake to above the RDA post-diet initiation. Iron intake decreased for 4 participants, remained the same for 1 participant, and increased for 9 participants post-diet initiation. Eleven participants were not meeting the RDA ( $8 \mathrm{mg}$ for men and $18 \mathrm{mg}$ for women ${ }^{145}$ ) for iron pre-diet initiation. Two additional participants' intakes fell below the RDA for iron post-diet initiation; however, 2 other participants' intakes increased to above the RDA for iron post-diet initiation. There were no statistically significant changes for micronutrients from baseline to week two of the low-FODMAP diet. A summary of changes in micronutrient intake is in table 3.

\section{Changes in Fiber}

Seven participants had a reduction in total fiber intake after starting the diet. For soluble and insoluble fiber, 11 and 6 participants had a reduction, respectively. At baseline, only 2 participants were above the daily fiber intake recommendation (14 grams per 1,000 calories consumed $^{145}$ ) and post-diet initiation only 1 participant was above the recommendation. None of the changes in fiber from baseline to week two were statistically significant, although there was a trend toward a significant reduction in soluble fiber $(\mathrm{p}=0.051)$. A summary of changes in fiber intake is in table 3 , and a comparison to recommendations for fiber is in table 4 . 


\section{Other Nutrients Evaluated}

There was a mean reduction in saturated fat and added sugar consumption for participants from baseline to week two of the low-FODMAP diet. However, intakes of saturated fat and added sugars were above recommendations for 8 and 4 participants post-diet initiation, respectively. A summary of changes in saturated fat and added sugar consumption is in table 3, and a comparison of participants' intake post-diet initiation to recommendations for these nutrients is in table 4 .

\section{Consumption from Food Groups}

There was a statistically significant reduction seen in grain $(\mathrm{p}=0.48)$ and dairy $(p=0.01)$ intake for participants. No other food groups were significantly reduced or increased after two weeks of the low-FODMAP diet. A depiction of the changes in food group intake post diet initiation is in figure 3.

\section{Compliance to the Diet}

Compliance to the diet varied. Ten (63\%) participants reported noncompliance to the diet at least once during the six-week period. Incidences of non-compliance ranged in occurrence from 1-24 days, with a mean of 2 days for all participants. The highest noncompliance rate was reported from week 4 to week 6 of the diet, with a mean of 1.4 days. From baseline to week 2 and from week 2 to week 4, patients reported a mean noncompliance of 1.2 and 1.1 days, Dairy products, wheat, garlic, and onion appear to be the largest contributors to non-compliance during the low-FODMAP diet and were consumed most frequently during the six-week intervention period. 


\section{Class Evaluation}

We received 45 completed class evaluations. Regarding preference of education session (group versus one-on-one), most class participants would prefer one-on-one education or stated the class format did not matter. The results to this question are highlighted in figure 4. Questions regarding session length, class content, class balance, and ability to ask questions were also on the class evaluation. For the session length, 42 thought it was "just right." Regarding the content of the class, 42 found the content "just right." For the balance of lecturing and group discussion, 44 found the balance "just right." It appears that most participants had the ability to discuss their concerns and were provided with enough information, with 39 and 30 responding yes to these questions, respectively.

\section{Discussion}

Numerous studies have been conducted regarding symptom relief for IBS patients after following the low-FODMAP diet, however the necessary length required for the elimination phase is undetermined. The statistically significant reduction in IBS-SSS by week two of the low-FODMAP diet suggests that it may only be necessary to follow the low-FODMAP diet elimination phase for two weeks in the majority of IBS patients who will clinically respond to the diet. Following the elimination phase for a shorter period of time would be beneficial for individuals since research has suggested potential nutritional inadequacy $^{123,125-126,136-137}$ and harm to the GI microbiome ${ }^{114,123,129,134}$ from following the diet. 
A reduction in energy intake has been seen in low-FODMAP diet studies previously. ${ }^{125-126,131,136}$ There was a reduction in energy intake seen in this study's participants, although several of the participants reported an energy intake below estimated requirements prior to diet initiation. Five participants were consuming less than $1200 \mathrm{kcal} /$ day while following the low-FODMAP diet, which makes it highly unlikely that they are able to meet all of their nutritional needs. Two of these 5 participants were already consuming less than $1200 \mathrm{kcal} /$ day at baseline. The reduction in energy intake post-diet initiation was not statistically significant; however, $36 \%(5 / 14)$ of participants were consuming a very low $(<1200 \mathrm{kcal} /$ day $)$ number of calories while following the low-FODMAP diet. Therefore, adequate energy intake during the low-FODMAP diet should remain a concern in future studies.

Only 3 participants were within the AMDR (45-65\% of calories ${ }^{144}$ ) for carbohydrates while following the low-FODMAP diet, and there was a statistically significant reduction in grams of carbohydrates consumed. Several randomized controlled trials have found a reduced the intake of carbohydrates during the low-FODMAP diet. $^{123,125-126,136}$ This result is of no surprise since the low-FODMAP diet aims to reduce carbohydrates that may cause symptoms for IBS patients. Current literature regarding the low-FODMAP diet states that fat and protein intake was unchanged after following the low-FODMAP diet, and our data was accordance with this. ${ }^{123,125-126}$ However, dietary fat intake was undesirably high for a majority of participants in this study. In the general IBS population, it is common to see an increased consumption of fat and a lower consumption of carbohydrates, ${ }^{80-81,147}$ which was displayed by participants in this study. 
Few studies have evaluated micronutrient intake while on the low-FODMAP diet. ${ }^{123,131,136-137}$ None of the changes in micronutrients from baseline to week two were statistically significant. Most participants were already below the RDA for vitamin C, vitamin $\mathrm{D}$, folate, calcium, and iron at baseline, prior to beginning the low-FODMAP diet. The largest micronutrient reduction post-diet initiation was in calcium intake. Staudacher et al. ${ }^{123}$ also found a lower calcium intake for subjects when compared to habitual diet, but a reduction was not seen in the study by Harvie et al. ${ }^{131}$ While there was a reduction in calcium intake for participants in this study, many of these participants were already below the RDA for calcium pre-diet initiation, and the reduction in calcium intake was not statistically significant. Staudacher et al. ${ }^{137}$ found a significant reduction in iron intake after following a low-FODMAP diet, but this study's results did not find a significant difference. Many participants' intake of iron actually increased in this study, which could possibly be due to an increased intake of meat.

Several RCTs have shown that fiber intake on a low-FODMAP diet does not differ when compared to controls. ${ }^{123,125-126}$ Only 1 participant met the recommendation for fiber while following the low-FODMAP diet (14 grams per 1,000 calories consumed ${ }^{145}$ ), and only 2 participants were meeting the recommendation for fiber at baseline. While this study did not find a statistically significant reduction in total dietary fiber intake after initiating the low-FODMAP diet, the low intake of fiber at baseline and week two of the low-FODMAP diet is concerning and possibilities to increase fiber intake should be evaluated. The reduction in soluble fiber was trending toward statistical significance $(\mathrm{p}=0.051)$. Beans and nuts are common high sources of soluble fiber, and 
some of these are restricted on the low-FODMAP diet, which could explain the greater reduction in soluble fiber.

While following the low-FODMAP diet, changes in food group intake is expected due to its restrictive nature of specific carbohydrates. Dairy products, grains, fruits, and vegetables are expected to be affected on the low-FODMAP diet since many of them are restricted. There was a statistically significant reduction in grains and dairy while following the low-FODMAP diet. Many commonly consumed grains, such as wheat, rye, and barely, are required to be avoided during the low-FODMAP diet elimination phase. A reduction in grains is concerning, because grains such as wheat, rye, and barley, are important sources of carbohydrates, fiber, B vitamins, and iron. ${ }^{151}$ Many dairy products, including milk, evaporated milk, and yogurt, are also required to be avoided during the elimination phase. A reduction in dairy is concerning because dairy products provide calcium, fat-soluble vitamins, and are fortified with vitamin D. ${ }^{150-151}$ The low-FODMAP education class that patients attended before participating in this study focused on proper replacement of foods, such as fruits and vegetables, to promote the consumption of a nutritionally adequate diet. Sufficient low-FODMAP diet education could be the reason why the intake of fruits and vegetables did not decrease during the intervention, and this emphasizes the importance of a low-FODMAP diet education to be led by a trained dietitian.

The average intake of lactose, fructose, and sugar alcohols all decreased from baseline to week two of the low-FODMAP diet, which supports participants were adhering to the diet. However, there were several incidences of non-compliance reported from participants. The most common high-FODMAP foods consumed while on the diet 
were dairy products, wheat, garlic, and onion. The poor compliance to the diet reported by some participants highlights the need to assess compliance in future interventions to determine if a reduction in IBS-SSS is a result of a reduction in FODMAP concentration or a placebo effect.

Of the 35 IBS patients who consented to the study, 14 (40\%) participants returned complete data and $2(6 \%)$ returned incomplete data. Nineteen (54\%) participants dropped out of the study. The low-FODMAP diet is very strict and cumbersome to follow. The literature has reported that common barriers in compliance to the diet are cost of alternative dietary products, low palatability of some speciality food products, and limited options for eating outside of home. ${ }^{9}$ This study found that an elimination phase of two weeks was statistically significant in promoting a clinical response to the diet. If patients are required to follow the diet for a shorter period of time, this could likely increase compliance and improve the dropout rate in future studies.

Most participants who completed the class evaluation were satisfied with the structure of the class. The literature has shown there is no significant difference in patient response to the low-FODMAP diet when provided with a group education session versus a one-on-one session. ${ }^{143}$ There is a limited supply of dietitians with appropriate expertise to counsel on the low-FODMAP $\operatorname{diet}^{9}{ }^{9}$ therefore a group education session is an appropriate alternative approach for dietitians with training on the low-FODMAP diet. Regardless of session type, is important that low-FODMAP dietary education is dietitianled. An improper delivery method of the low-FODMAP diet could lead to incomplete FODMAP restriction or an over-restrictive dietary intake, which would lead to lack of efficiency or nutritional inadequacies, respectively. ${ }^{9}$ 
Randomized controlled trials are the gold standard for clinical studies. Our study lacks a control group to support that the results of the intervention were truly due to the intervention performed. Our study also lacks blinding, however, since the intervention in this study was a change in dietary pattern, the opportunity for participant blinding was not possible. There were some major limitations of this study. One limitation of this study is its sample size. A smaller sample size can affect a study's power and cause an inability to perform statistical analysis. While this study did find statistical significance for some variables, it is possible that there may be significant differences in other variables that were not found due to a low power. Another major limitation in this study is using patient-reported data and being forced to rely on participants to return complete data. Efforts were made to minimize patient burden through providing envelopes, stamps, and reminder calls or texts; however, some participants did not return any documents or did not include all documents in their returned data. With patient reported data, there is a risk of bias regarding the data that is returned. There is also the risk of reactivity when participants are completing the required study instrumentation.

\section{Conclusion}

The findings of this study suggest a low-FODMAP diet elimination phase of two weeks appears optimal for achieving symptom relief. Due to the restrictive nature of the low-FODMAP diet, an inadequate intake of nutrients and deficiencies in micronutrients are possible, although there is limited research in this area. There was a significant reduction in grain and dairy intake, which could affect the overall consumption of both macro- and micronutrients. There was also a lower carbohydrate consumption after 
beginning the low-FODMAP diet for participants in this study. Moreover, the lowFODMAP diet may lead to a nutritionally inadequate dietary pattern and must be monitored in future studies. A shorter total elimination period would help prevent nutritional inadequacies and could improve patient compliance. The poor compliance reported in this study highlights the need to assess compliance in future interventions to determine if a reduction in IBS-SSS is a result of FODMAP concentration or placebo effect. This study proposes that a proper dietitian led education session can help adequate nutritional intake by educating patients on proper substitutions of FODMAP containing foods, and a group education session is suitable for this dietary intervention. More rigourous studies with a larger sample size are necessary to evaluate nutritional adequacy and compliance with the low-FODMAP diet. 


\section{Tables and Figures}

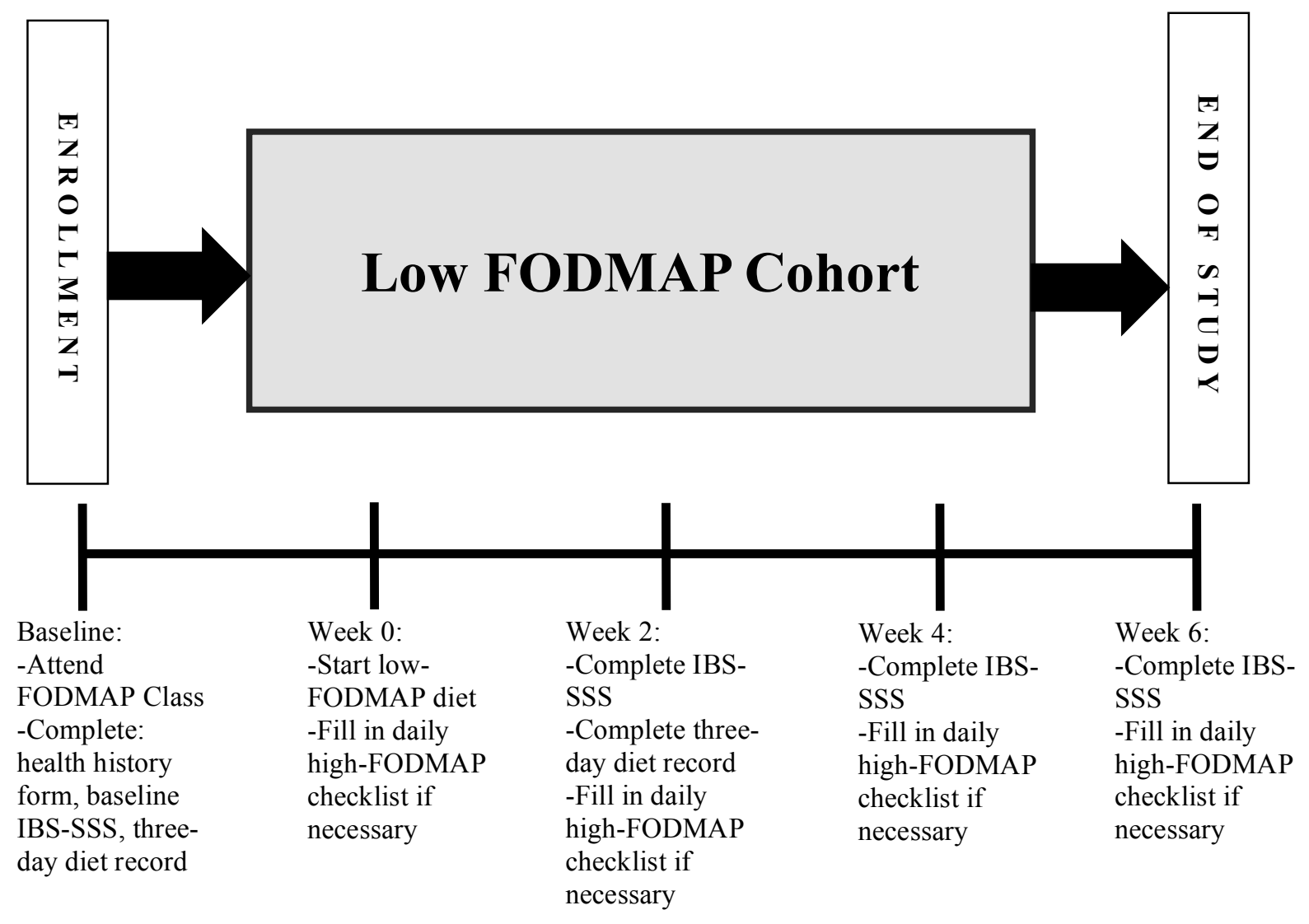

Figure 19. Study Intervention 


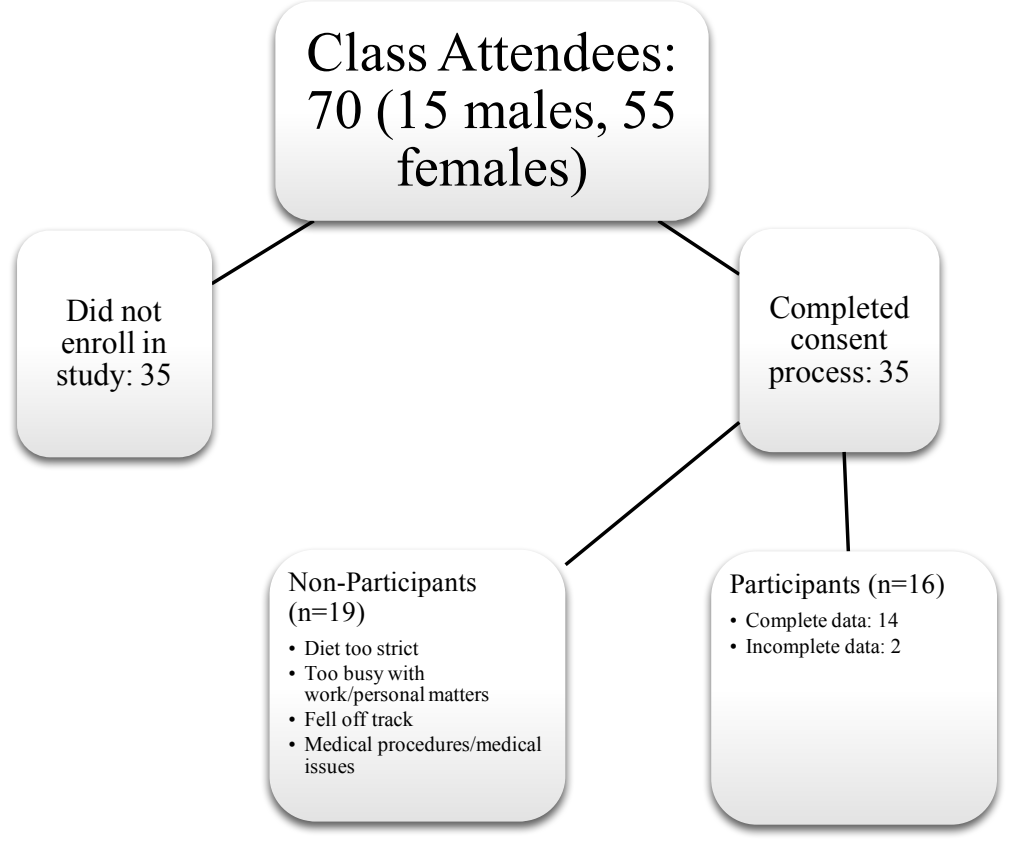

Figure 20. Study Enrollment

\section{Characteristic}

Age (years), mean, SD

Age range (years)

Sex

Females, frequency, \%

Males, frequency, \%

Body Mass Index $\left(\mathrm{kg} / \mathrm{m}^{2}\right)$, mean, SD

BMI range $\left(\mathrm{kg} / \mathrm{m}^{2}\right)$

Physically Active, \%

Smoker, \%

Alcohol Use, \%
Total $(\mathbf{n}=35)$

$47.7,16.3$

21-74

27,77

8,23

$28.7,8.0$

16.9-54.6

80

8.5

43
Participants $(\mathbf{n}=16)$

$44.1,15.7$

21-70

$10,62.5$

$6,37.5$

$28.7,9.2$

18.3-54.6

75

0

50

Table 9. Demographic and Health History Results for Patients with Irritable Bowel

Syndrome 


\begin{tabular}{|c|c|c|c|c|c|}
\hline Week & $\begin{array}{c}\text { Mean IBS- } \\
\text { SSS Score } \\
\quad(n=16)\end{array}$ & $\begin{array}{l}\text { Standard } \\
\text { Deviation }\end{array}$ & $\begin{array}{c}\text { P-value } \\
\text { from } \\
\text { Baseline }\end{array}$ & $\begin{array}{l}\text { Degrees of } \\
\text { Freedom }\end{array}$ & $\mathbf{F}$ \\
\hline Baseline & 310.8 & 90.8 & & \multirow[t]{4}{*}{3} & \multirow[t]{4}{*}{19.997} \\
\hline Week 2 & 168.8 & 107.0 & $<0.001$ & & \\
\hline Week 4 & 172.6 & 106.4 & 0.002 & & \\
\hline Week 6 & 146.2 & 105.5 & $<0.001$ & & \\
\hline
\end{tabular}

Table 10. Analysis of IBS-SSS Data $(n=16)$

\begin{tabular}{|c|c|c|c|}
\hline Variable & $\begin{array}{c}\text { Mean Intake for } \\
\text { All Participants at } \\
\text { Week } 2\end{array}$ & $\begin{array}{c}\text { Change from } \\
\text { Baseline to } \\
\text { Week } 2\end{array}$ & P-value \\
\hline Kcal $($ mean \pm SD) & 1484 & $-244.5 \pm 667.8$ & .194 \\
\hline CHO, grams (mean \pm SD) & 141 & $-50.2 \pm 77.9$ & $.031 *$ \\
\hline $\begin{array}{l}\text { CHO, \% total kcal (mean } \pm \\
\text { SD) }\end{array}$ & 38.7 & $-4.0 \pm 9.3$ & .129 \\
\hline Protein, grams (mean \pm SD) & 69 & $-1.4 \pm 32.8$ & .873 \\
\hline $\begin{array}{l}\text { Protein, \% total kcal (mean } \pm \\
\text { SD) }\end{array}$ & 18.7 & $1.6 \pm 5.4$ & .290 \\
\hline Fat, grams (mean \pm SD) & 65 & $-11.8 \pm 34.9$ & .229 \\
\hline Fat, \% total kcal (mean \pm SD) & 38.2 & $-.79 \pm 6.0$ & .630 \\
\hline $\begin{array}{l}\text { SFA, \% total kcal (mean } \pm \\
\text { SD) }\end{array}$ & 12.1 & $-1.4 \pm 3.7$ & .195 \\
\hline $\begin{array}{l}\text { Added Sugars, \% total kcal } \\
\text { (mean } \pm \text { SD) }\end{array}$ & 8 & $-3.5 \pm 5.1$ & .24 \\
\hline Vitamin $D$, mcg $($ mean \pm SD) & 8.1 & $1.4 \pm 3.4$ & .16 \\
\hline Vitamin C, mg (mean \pm SD) & 61.8 & $9.8 \pm 32.9$ & .286 \\
\hline Folate, mcg (mean \pm SD) & 248.1 & $-16.3 \pm 114.6$ & .604 \\
\hline Calcium, mg (mean \pm SD) & 636.9 & $-173.9 \pm 407.3$ & .134 \\
\hline Iron, mg (mean \pm SD) & 11 & $.47 \pm 5.3$ & .745 \\
\hline Fiber, grams (mean \pm SD) & 14.3 & $-.22 \pm 5.2$ & .876 \\
\hline $\begin{array}{l}\text { Soluble Fiber, grams (mean } \pm \\
\text { SD) }\end{array}$ & 3.8 & $-1.2 \pm 2.1$ & .051 \\
\hline $\begin{array}{l}\text { Insoluble Fiber, grams (mean } \\
\pm \text { SD) }\end{array}$ & 10.2 & $.79 \pm 3.5$ & .422 \\
\hline Fruit Servings & 1 & $-.04 \pm .81$ & .872 \\
\hline
\end{tabular}

Table 11. Analysis of Nutrient Intake Post Low-FODMAP Diet Initiation $(n=14)$ 


\begin{tabular}{|l|c|c|c|}
\hline Vegetable Servings & 3.6 & $1.1 \pm 2.2$ & .872 \\
\hline Grain Servings & 3.4 & $-2.3 \pm 3.9$ & $.048^{*}$ \\
\hline Meat Servings & 6.7 & $.99 \pm 2.8$ & .214 \\
\hline Dairy Servings & 1.7 & $-1.0 \pm 1.3$ & $.010^{*}$ \\
\hline Fat Servings & 3 & $-.17 \pm 1.9$ & .743 \\
\hline Dessert Servings & 1.7 & $-.95 \pm 2.2$ & .133 \\
\hline
\end{tabular}

...Continued

\begin{tabular}{|c|c|c|c|c|}
\hline Nutrient & $\begin{array}{c}\text { Recommended } \\
\text { Range }\end{array}$ & $\begin{array}{c}\text { Met } \\
\text { Recommendation } \\
\text { (n) }\end{array}$ & $\begin{array}{c}\text { Below } \\
\text { Recommendation } \\
\text { (n) }\end{array}$ & $\begin{array}{c}\text { Exceeded } \\
\text { Recommendation } \\
\text { (n) }\end{array}$ \\
\hline $\begin{array}{c}\text { Calories } \\
(\text { kcal/day })^{1}\end{array}$ & Varies & 1 & 8 & 5 \\
\hline $\begin{array}{l}\text { Protein } \\
(\% \text { kcal })^{2}\end{array}$ & $10-35 \%$ & 13 & 1 & 0 \\
\hline $\begin{array}{c}\text { Carbs } \\
(\% \text { kcal })^{2}\end{array}$ & $45-65 \%$ & 3 & 11 & 0 \\
\hline $\begin{array}{c}\text { Fat } \\
(\% \text { kcal })^{2}\end{array}$ & $20-35 \%$ & 5 & 0 & 9 \\
\hline $\begin{array}{c}\text { Saturated } \\
\text { Fat } \\
(\% \text { kcal })^{3}\end{array}$ & $<10 \%$ & 6 & $\mathrm{~N} / \mathrm{A}$ & 8 \\
\hline $\begin{array}{l}\text { Added } \\
\text { Sugars } \\
\text { (\%kcal) }\end{array}$ & $<10 \%$ & 10 & $\mathrm{~N} / \mathrm{A}$ & 4 \\
\hline Fiber (g) & Varies & 1 & 13 & N/A \\
\hline \multicolumn{5}{|c|}{$\begin{array}{l}\text { * Recommendations include the following: } \\
\text { 1. Estimated daily energy requirements for weight maintenance }(20-25 \mathrm{kcal} / \mathrm{kg} / \mathrm{d}) \\
\text { 2. Acceptable Macronutrient Distribution Range (AMDR) set by the Institute of Medicine } \\
\text { (IOM) } \\
\text { 3. Recommendations from the } 2015-2020 \text { Dietary Guidelines for Americans }{ }^{145}\end{array}$} \\
\hline Table 12. Dis & ibution of Part & pants Meeting $N$ & rient Recommends & ions Post Low- \\
\hline
\end{tabular}




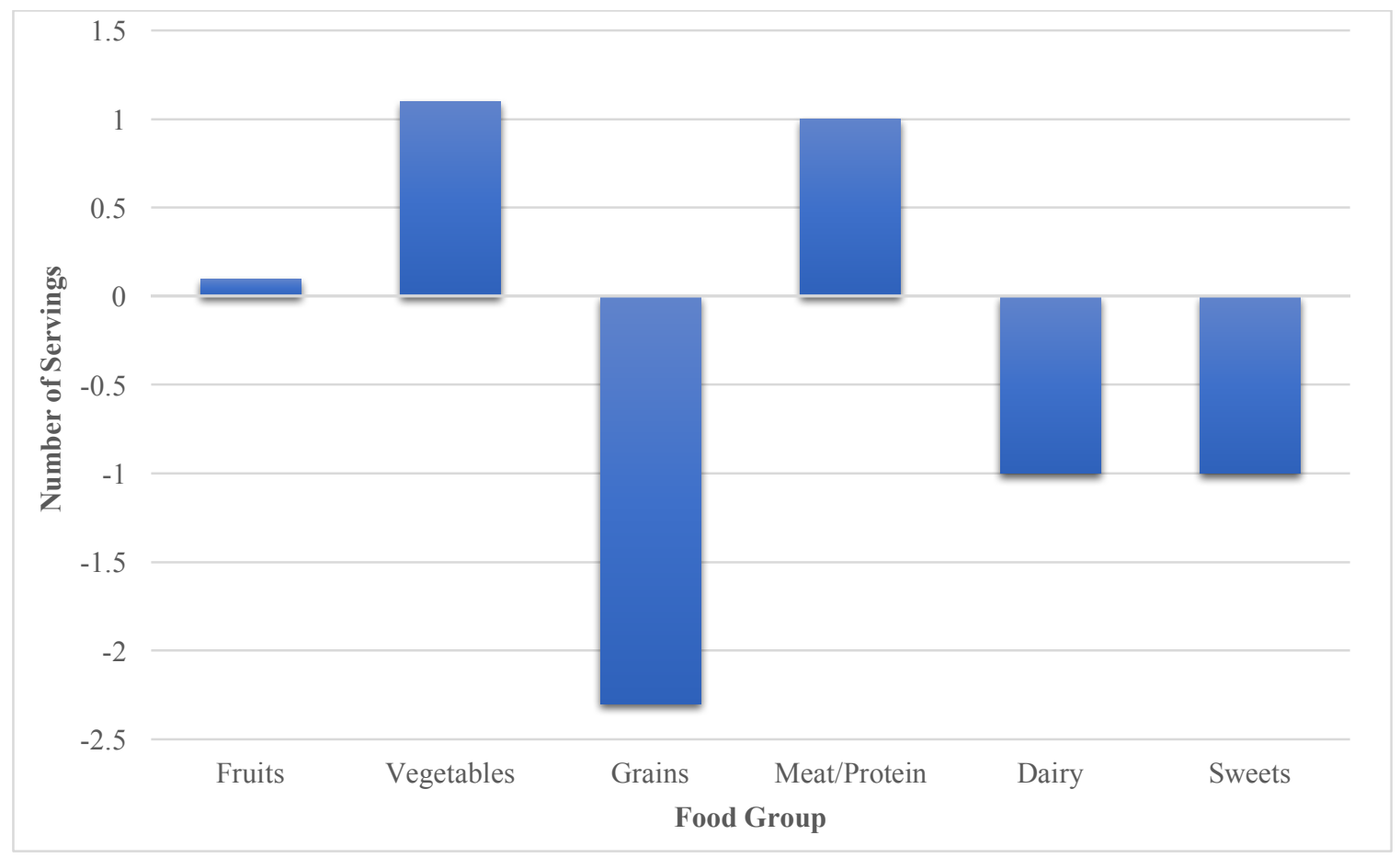

Figure 21. Mean Change in Food Group Servings $(n=14)$

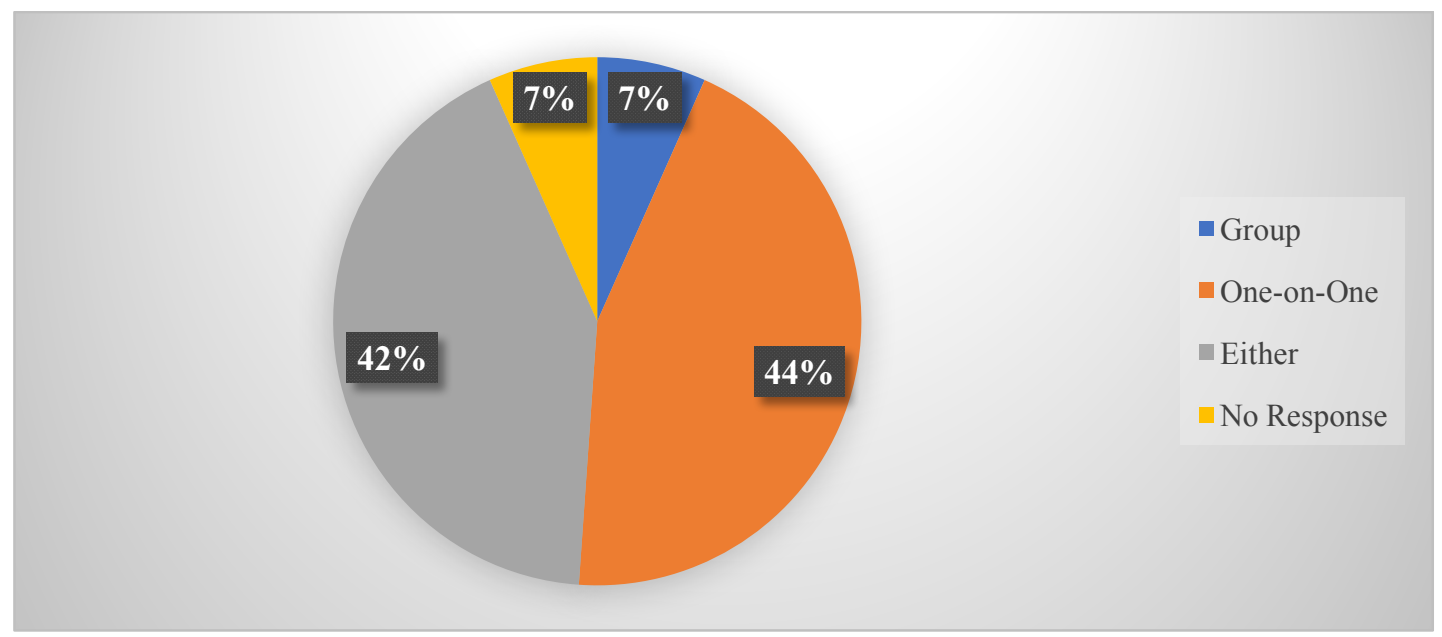

Figure 22. Preferred Dietary Education Session Feedback from Class Evaluation 


\section{Bibliography}

1. Longstreth GF, Thompson WG, Chey WD, Houghton LA, Mearin F, Spiller RC. Functional Bowel Disorders. Gastroenterology. 2006;130(5):1480-1491. doi:10.1053/j.gastro.2005.11.061

2. Hayes P, Corish C, O’Mahony E, Quigley EMM. A dietary survey of patients with irritable bowel syndrome. J Hum Nutr Diet. 2014;27:36-47. doi:10.1111/jhn.12114

3. Simrén M, Månsson A, Langkilde AM, et al. Food-related gastrointestinal symptoms in the irritable bowel syndrome. Digestion. 2001;63(2):108-115. doi:51878

4. Heizer WD, Southern S, McGovern S. The Role of Diet in Symptoms of Irritable Bowel Syndrome in Adults: A Narrative Review. J Am Diet Assoc. 2009;109(7):1204-1214. doi:10.1016/j.jada.2009.04.012

5. Ford AC, Moayyedi P, Lacy BE, et al. American College of Gastroenterology Monograph on the Management of Irritable Bowel Syndrome and Chronic Idiopathic Constipation. Am J Gastroenterol. 2014;109:S2-S26. doi:10.1038/ajg.2014.187

6. Staudacher HM. Nutritional, microbiological and psychosocial implications of the low FODMAP diet: Implications of the low FODMAP diet. $J$ Gastroenterol Hepatol. 2017;32:16-19. doi:10.1111/jgh.13688

7. Sood R, Law GR, Ford AC. Diagnosis of IBS: symptoms, symptom-based criteria, biomarkers or "psychomarkers"? Nat Rev Gastroenterol Hepatol. 2014;11(11):683691.

8. Gibson PR. The evidence base for efficacy of the low FODMAP diet in irritable bowel syndrome: is it ready for prime time as a first-line therapy?: Efficacy of the low FODMAP diet. J Gastroenterol Hepatol. 2017;32:32-35. doi:10.1111/jgh.13693

9. Whelan K, Martin LD, Staudacher HM, Lomer MCE. The low FODMAP diet in the management of irritable bowel syndrome: an evidence-based review of FODMAP restriction, reintroduction and personalisation in clinical practice. J Hum Nutr Diet. January 2018. doi:10.1111/jhn. 12530 
10. Staudacher HM, Whelan K, Irving PM, Lomer MCE. Comparison of symptom response following advice for a diet low in fermentable carbohydrates (FODMAPs) versus standard dietary advice in patients with irritable bowel syndrome: IBS symptom response to a low FODMAP diet. J Hum Nutr Diet. 2011;24(5):487-495. doi:10.1111/j.1365-277X.2011.01162.x

11. TheFreeDictionary.com. Medical Dictionary. http://medicaldictionary.thefreedictionary.com. Accessed August 15, 2017.

12. Lisa M Baumgartner, Lee MY, Birden S, Flowers D. Adult Learning Theory: A Primer. http://files.eric.ed.gov/fulltext/ED482337.pdf. Accessed August 29, 2017.

13. Hungin APS, Chang L, Locke GR, Dennis EH, Barghout V. Irritable bowel syndrome in the United States: prevalence, symptom patterns and impact. Aliment Pharmacol Ther. 2005;21(11):1365-1375. doi:10.1111/j.1365-2036.2005.02463.x

14. Card T, Canavan C, West J. The epidemiology of irritable bowel syndrome. Clin Epidemiol. February 2014:71. doi:10.2147/CLEP.S40245

15. Inadomi JM, Fennerty MB, Bjorkman D. Systematic review: the economic impact of irritable bowel syndrome. Aliment Pharmacol Ther. 2003;18(7):671-682.

16. Kellow JE. The "Pro" case. The Rome III criteria. Neurogastroenterol Motil. 2007;19(10):787-792. doi:10.1111/j.1365-2982.2007.01002.x

17. Brandt LJ, Chey WD, Foxx-Orenstein AE, et al. An Evidence-Based Position Statement on the Management of Irritable Bowel Syndrome. Am J Gastroenterol. 2008;104:S1-S35. doi:10.1038/ajg.2008.122

18. Lovell RM, Ford AC. Global Prevalence of and Risk Factors for Irritable Bowel Syndrome: A Meta-analysis. Clin Gastroenterol Hepatol. 2012;10(7):712-721.e4. doi:10.1016/j.cgh.2012.02.029

19. Horwitz BJ, Fisher RS. The Irritable Bowel Syndrome. $N$ Engl J Med. 2001;344(24):1846-1850. doi:10.1056/NEJM200106143442407

20. El-Serag HB, Olden K, Bjorkman D. Health-related quality of life among persons with irritable bowel syndrome: a systematic review. Aliment Pharmacol Ther. 2002;16(6):1171-1185.

21. Distrutti E, Monaldi L, Ricci P, Fiorucci S. Gut microbiota role in irritable bowel syndrome: New therapeutic strategies. World J Gastroenterol. 2016;22(7):22192241.

22. Drossman DA. The Functional Gastrointestinal Disorders and the Rome III Process. Gastroenterology. 2006;130(5):1377-1390. doi:10.1053/j.gastro.2006.03.008 
23. Tanaka Y, Kanazawa M, Fukudo S, Drossman DA. Biopsychosocial Model of Irritable Bowel Syndrome. J Neurogastroenterol Motil. 2011;17(2):131-139. doi:10.5056/jnm.2011.17.2.131

24. Sperber AD, Dumitrascu D, Fukudo S, et al. The global prevalence of IBS in adults remains elusive due to the heterogeneity of studies: a Rome Foundation working team literature review. Gut. 2017;66(6):1075-1082. doi:10.1136/gutjnl-2015-311240

25. Ballou S, Keefer L. The impact of irritable bowel syndrome on daily functioning: Characterizing and understanding daily consequences of IBS. Neurogastroenterol Motil. 2017;29(4):e12982. doi:10.1111/nmo.12982

26. Parkes GC, Brostoff J, Whelan K, Sanderson JD. Gastrointestinal Microbiota in Irritable Bowel Syndrome: Their Role in Its Pathogenesis and Treatment. Am J Gastroenterol. 2008;103(6):1557-1567. doi:10.1111/j.1572-0241.2008.01869.x

27. Lee OY, Mayer EA, Schmulson M, Chang L, Naliboff B. Gender-related differences in IBS symptoms. Am J Gastroenterol. 2001;96(7):2184-2193.

28. Neal KR, Barker L, Spiller RC. Prognosis in post-infective irritable bowel syndrome: a six year follow up study. Gut. 2002;51(3):410-413.

29. Sood R, Law GR, Ford AC. Diagnosis of IBS: symptoms, symptom-based criteria, biomarkers or "psychomarkers"? Nat Rev Gastroenterol Hepatol. 2014;11(11):683691. doi:10.1038/nrgastro.2014.127

30. Manning AP, Thompson WG, Heaton KW, Morris AF. Towards positive diagnosis of the irritable bowel. Br Med J. 1978;2(6138):653-654.

31. Simren M, Palsson OS, Whitehead WE. Update on Rome IV Criteria for Colorectal Disorders: Implications for Clinical Practice. Curr Gastroenterol Rep. 2017;19(4). doi:10.1007/s11894-017-0554-0

32. Lewis SJ, Heaton KW. Stool form scale as a useful guide to intestinal transit time. Scand J Gastroenterol. 1997;32(9):920-924. doi:10.3109/00365529709011203

33. Francis CY, Morris J, Whorwell PJ. The irritable bowel severity scoring system: a simple method of monitoring irritable bowel syndrome and its progress. Aliment Pharmacol Ther. 1997;11(2):395-402. doi:10.1046/j.1365-2036.1997.142318000.x

34. Hungin APS, Becher A, Cayley B, et al. Irritable bowel syndrome: an integrated explanatory model for clinical practice. Neurogastroenterol Motil. 2015;27(6):750763. doi:10.1111/nmo.12524

35. Lee KN, Lee OY. The Role of Mast Cells in Irritable Bowel Syndrome. Gastroenterol Res Pract. 2016;2016:1-11. doi:10.1155/2016/2031480 
36. Kim D-Y, Camilleri M. Serotonin: a mediator of the brain-gut connection. $A m J$ Gastroenterol. 2000;95(10):2698-2709. doi:10.1111/j.1572-0241.2000.03177.x

37. Ohman L, Simren M. New insights into the pathogenesis and pathophysiology of irritable bowel syndrome. Dig Liver Dis. 2007;39(3):201-215. doi:10.1016/j.dld.2006.10.014

38. Bearcroft CP, Farthing MJG, Perrett D. Postprandial plasma 5-hydroxytryptamine in diarrhoea predominant irritable bowel syndrome: a pilot study. GUT. 1998;42(9801):42.

39. Cremon C, Carini G, Wang B, et al. Intestinal Serotonin Release, Sensory Neuron Activation, and Abdominal Pain in Irritable Bowel Syndrome. Am J Gastroenterol. 2011;106(7):1290-1298. doi:10.1038/ajg.2011.86

40. El-Salhy M. Recent developments in the pathophysiology of irritable bowel syndrome. World J Gastroenterol. 2015;21(25):7621-7636. doi:10.3748/wjg.v21.i25.7621

41. El-Salhy M, Vaali K, Dizdar V, Hausken T. Abnormal Small-Intestinal Endocrine Cells in Patients with Irritable Bowel Syndrome. Dig Dis Sci. 2010;55(12):35083513. doi:10.1007/s10620-010-1169-6

42. El-Salhy M, Gundersen D, Hatlebakk JG, Gilja OH, Hausken T. Abnormal rectal endocrine cells in patients with irritable bowel syndrome. Regul Pept. 2014;188:6065. doi:10.1016/j.regpep.2013.11.005

43. El-Salhy M, Gilja OH, Gundersen D, Hatlebakk JG, Hausken T. Duodenal Chromogranin A Cell Density as a Biomarker for the Diagnosis of Irritable Bowel Syndrome. Gastroenterol Res Pract. 2014;2014:1-8. doi:10.1155/2014/462856

44. Stanghellini V, Tosetti C, Barbara G, et al. Dyspeptic symptoms and gastric emptying in the irritable bowel syndrome. Am J Gastroenterol. 2002;97(11):2738-2743.

45. Hayes PA, Fraher MH, Quigley EM. Irritable bowel syndrome: the role of food in pathogenesis and management. Gastroenterol Hepatol. 2014;10(3):164-174.

46. Camilleri M. Peripheral Mechanisms in Irritable Bowel Syndrome. $N$ Engl J Med. 2012;367(17):1626-1635. doi:10.1056/NEJMra1207068

47. Wedlake L, A'Hern R, Russell D, Thomas K, Walters JRF, Andreyev HJN. Systematic review: the prevalence of idiopathic bile acid malabsorption as diagnosed by SeHCAT scanning in patients with diarrhoea-predominant irritable bowel syndrome. Aliment Pharmacol Ther. 2009;30(7):707-717. doi:10.1111/j.13652036.2009.04081.x 
48. Öhman L, Simrén M. Pathogenesis of IBS: role of inflammation, immunity and neuroimmune interactions. Nat Rev Gastroenterol Hepatol. 2010;7(3):163-173. doi:10.1038/nrgastro.2010.4

49. Nelms M, Sucher K, Lacey K. Nutrition Therapy and Pathophysiology. 3rd ed. Cengage Learning; 2016.

50. Barbara G, Stanghellini V, De Giorgio R, et al. Activated mast cells in proximity to colonic nerves correlate with abdominal pain in irritable bowel syndrome. Gastroenterology. 2004;126(3):693-702. doi:10.1053/j.gastro.2003.11.055

51. Barbara G, Stanghellini V, De Giorgio R, Corinaldesi R. Functional gastrointestinal disorders and mast cells: implications for therapy. Neurogastroenterol Motil Off J Eur Gastrointest Motil Soc. 2006;18(1):6-17.

52. Spiller R, Garsed K. Postinfectious Irritable Bowel Syndrome. YGAST Gastroenterol. 2009;136(6):1979-1988.

53. Gonsalkorale WM, Perrey C, Pravica V, Whorwell PJ, Hutchinson IV. Interleukin 10 genotypes in irritable bowel syndrome: evidence for an inflammatory component? Gut. 2003;52(1):91-93.

54. Kassinen A, Krogius-Kurikka L, Mäkivuokko H, et al. The Fecal Microbiota of Irritable Bowel Syndrome Patients Differs Significantly From That of Healthy Subjects. Gastroenterology. 2007;133(1):24-33. doi:10.1053/j.gastro.2007.04.005

55. Pimentel M, Chow EJ, Lin HC. Eradication of small intestinal bacterial overgrowth reduces symptoms of irritable bowel syndrome. Am J Gastroenterol. 2000;95(12):3503-3506. doi:10.1111/j.1572-0241.2000.03368.x

56. Ford AC, Spiegel BMR, Talley NJ, Moayyedi P. Small Intestinal Bacterial Overgrowth in Irritable Bowel Syndrome: Systematic Review and Meta-analysis. Clin Gastroenterol Hepatol. 2009;7(12):1279-1286. doi:10.1016/j.cgh.2009.06.031

57. Bohm M, Siwiec RM, Wo JM. Diagnosis and Management of Small Intestinal Bacterial Overgrowth. Nutr Clin Pract. 2013;28(3):289-299. doi: $10.1177 / 0884533613485882$

58. Ghoshal UC, Shukla R, Ghoshal U. Small Intestinal Bacterial Overgrowth and Irritable Bowel Syndrome: A Bridge between Functional Organic Dichotomy. Gut Liver. 2017;11(2):196-208. doi:10.5009/gnl16126

59. Pimentel M. Normalization of lactulose breath testing correlates with symptom improvement in irritable bowel syndrome a double-blind, randomized, placebocontrolled study. Am J Gastroenterol. 2003;98(2):412-419. doi:10.1016/S00029270(02)05902-6 
60. Spiegel BMR. Questioning the Bacterial Overgrowth Hypothesis of Irritable Bowel Syndrome: An Epidemiologic and Evolutionary Perspective. Clin Gastroenterol Hepatol. 2011;9(6):461-469. doi:10.1016/j.cgh.2011.02.030

61. Camilleri M. Visceral hypersensitivity: facts, speculations, and challenges. Gut. 2001;48(1):125-131. doi:10.1136/gut.48.1.125

62. Gibson PR, Shepherd SJ. Evidence-based dietary management of functional gastrointestinal symptoms: The FODMAP approach. J Gastroenterol Hepatol. 2010;25(2):252-258. doi:10.1111/j.1440-1746.2009.06149.x

63. Krogsgaard LR, Lyngesen M, Bytzer P. Systematic review: quality of trials on the symptomatic effects of the low FODMAP diet for irritable bowel syndrome. Aliment Pharmacol Ther. 2017;45(12):1506-1513. doi:10.1111/apt.14065

64. Houghton LA, Lea R, Jackson N, Whorwell PJ. The menstrual cycle affects rectal sensitivity in patients with irritable bowel syndrome but not healthy volunteers. Gut. 2002;50(4):471-474.

65. Murray CDR, Flynn J, Ratcliffe L, Jacyna MR, Kamm MA, Emmanuel AV. Effect of acute physical and psychological stress on gut autonomic innervation in irritable bowel syndrome. Gastroenterology. 2004;127(6):1695-1703. doi:10.1053/j.gastro.2004.08.057

66. Gupta V, Sheffield D, Verne GN. Evidence for Autonomic Dysregulation in the Irritable Bowel Syndrome. Dig Sci Dig Dis Sci. 2002;47(8):1716-1722.

67. van Orshoven NP, Andriesse GI, van Schelven LJ, Smout AJ, Akkermans LMA, Oey PL. Subtle involvement of the parasympathetic nervous system in patients with irritable bowel syndrome. Clin Auton Res. 2006;16(1):33-39. doi:10.1007/s10286006-0307-x

68. Lea R, Whorwell PJ. New insights into the psychosocial aspects of irritable bowel syndrome. Curr Gastroenterol Rep Curr Gastroenterol Rep. 2003;5(4):343-350.

69. Drossman DA. Do psychosocial factors define symptom severity and patient status in irritable bowel syndrome? Am J Med. 1999;107(5):41-50. doi:10.1016/S00029343(99)00081-9

70. Spiller R. Review article: probiotics and prebiotics in irritable bowel syndrome. Aliment Pharmacol Ther. 2008;28(4):385-396. doi:10.1111/j.13652036.2008.03750.x

71. Cauffield JS. The psychosocial aspects of complementary and alternative medicine. Pharmacotherapy. 2000;20(11):1289-1294. 
72. National Center for Complementary and Integrative Health. Complementary, Alternative, or Integrative Health: What's In a Name?

https://nccih.nih.gov/health/integrative-health\#cvsa. Accessed February 21, 2018.

73. van Tilburg MA, Palsson OS, Levy RL, et al. Complementary and alternative medicine use and cost in functional bowel disorders: A six month prospective study in a large HMO. BMC Complement Altern Med. 2008;8(1). doi:10.1186/1472-6882$8-46$

74. Merat S, Khalili S, Mostajabi P, Ghorbani A, Ansari R, Malekzadeh R. The Effect of Enteric-Coated, Delayed-Release Peppermint Oil on Irritable Bowel Syndrome. Dig Dis Sci. 2010;55(5):1385-1390. doi:10.1007/s10620-009-0854-9

75. Grigoleit H-G, Grigoleit P. Peppermint oil in irritable bowel syndrome. Phytomedicine. 2005;12(8):601-606. doi:10.1016/j.phymed.2004.10.005

76. Liu J-H, Chen G-H, Yeh H-Z, Huang C-K, Poon S-K. Enteric-coated peppermint-oil capsules in the treatment of irritable bowel syndrome: A prospective, randomized trial. J Gastroenterol J Gastroenterol. 1997;32(6):765-768.

77. Monsbakken KW, Vandvik PO, Farup PG. Perceived food intolerance in subjects with irritable bowel syndrome - etiology, prevalence and consequences. Eur J Clin Nutr. 2006;60(5):667-672. doi:10.1038/sj.ejcn.1602367

78. Crowell MD, Cheskin LJ, Musial F. Prevalence of gastrointestinal symptoms in obese and normal weight binge eaters. Am J Gastroenterol. 1994;89(3):387-391.

79. Cremonini F, Camilleri M, Clark MM, et al. Associations among binge eating behavior patterns and gastrointestinal symptoms: a population-based study. Int $J$ Obes. 2009;33(3):342-353. doi:10.1038/ijo.2008.272

80. Tigchelaar EF, Mujagic Z, Zhernakova A, et al. Habitual diet and diet quality in Irritable Bowel Syndrome: A case-control study. Neurogastroenterol Motil. 2017;29(12):e13151. doi:10.1111/nmo.13151

81. Prescha A, Pieczyńska J, Ilow R, et al. Assessment of dietary intake of patients with irritable bowel syndrome. Rocz Panstw Zakl Hig. 2009;60(2):185-189.

82. Atkinson W. Food elimination based on IgG antibodies in irritable bowel syndrome: a randomised controlled trial. Gut. 2004;53(10):1459-1464.

doi:10.1136/gut.2003.037697

83. Young E, Stoneham MD, Petruckevitch A, Barton J, Rona R. A population study of food intolerance. Lancet Lond Engl. 1994;343(8906):1127-1130.

84. Gearry RB, Irving PM, Barrett JS, Nathan DM, Shepherd SJ, Gibson PR. Reduction of dietary poorly absorbed short-chain carbohydrates (FODMAPs) improves 
abdominal symptoms in patients with inflammatory bowel disease - a pilot study. $J$ Crohns Colitis. 2009;3(1):8-14. doi:10.1016/j.crohns.2008.09.004

85. Biesiekierski JR, Newnham ED, Irving PM, et al. Gluten Causes Gastrointestinal Symptoms in Subjects Without Celiac Disease: A Double-Blind Randomized Placebo-Controlled Trial. Am J Gastroenterol. 2011;106(3):508-514. doi:10.1038/ajg.2010.487

86. Wahnschaffe U, Schulzke J, Zeitz M, Ullrich R. Predictors of Clinical Response to Gluten-Free Diet in Patients Diagnosed With Diarrhea-Predominant Irritable Bowel Syndrome. Clin Gastroenterol Hepatol. 2007;5(7):844-850. doi:10.1016/j.cgh.2007.03.021

87. Vazquez-Roque MI, Camilleri M, Smyrk T, et al. A Controlled Trial of Gluten-Free Diet in Patients With Irritable Bowel Syndrome-Diarrhea: Effects on Bowel Frequency and Intestinal Function. Gastroenterology. 2013;144(5):903-911.e3. doi:10.1053/j.gastro.2013.01.049

88. Aziz I, Trott N, Briggs R, North JR, Hadjivassiliou M, Sanders DS. Efficacy of a Gluten-Free Diet in Subjects With Irritable Bowel Syndrome-Diarrhea Unaware of Their HLA-DQ2/8 Genotype. Clin Gastroenterol Hepatol. 2016;14(5):696-703.e1. doi:10.1016/j.cgh.2015.12.031

89. Bijkerk CJ, Muris JWM, Knottnerus JA, Hoes AW, De Wit NJ. Systematic review: the role of different types of fibre in the treatment of irritable bowel syndrome. Aliment Pharmacol Ther. 2004;19(3):245-251. doi:10.1111/j.02692813.2004.01862.x

90. McRorie JW. Evidence-Based Approach to Fiber Supplements and Clinically Meaningful Health Benefits, Part 2: What to Look for and How to Recommend an Effective Fiber Therapy. Nutr Today. 2015;50(2):90-97. doi:10.1097/NT.0000000000000089

91. Bijkerk CJ, de Wit NJ, Muris JWM, Whorwell PJ, Knottnerus JA, Hoes AW. Soluble or insoluble fibre in irritable bowel syndrome in primary care? Randomised placebo controlled trial. BMJ. 2009;339(aug27 2):b3154-b3154. doi:10.1136/bmj.b3154

92. Suchy FJ, Brannon PM, Carpenter TO, et al. National Institutes of Health Consensus Development Conference: lactose intolerance and health. Ann Intern Med. 2010;152(12):792-796.

93. Yang J, Deng Y, Chu H, et al. Prevalence and Presentation of Lactose Intolerance and Effects on Dairy Product Intake in Healthy Subjects and Patients With Irritable Bowel Syndrome. Clin Gastroenterol Hepatol. 2013;11(3):262-268.e1. doi:10.1016/j.cgh.2012.11.034 
94. Parker TJ, Woolner JT, Prevost AT, Tuffnell Q, Shorthouse M, Hunter JO. Irritable bowel syndrome: is the search for lactose intolerance justified? Eur J Gastroenterol Hepatol. 2001;13(3):219-225.

95. Pimentel M. Breath testing to evaluate lactose intolerance in irritable bowel syndrome correlates with lactulose testing and may not reflect true lactose malabsorption. Am J Gastroenterol. 2003;98(12):2700-2704. doi:10.1016/S00029270(03)01703-9

96. Turnbull GK. Lactose intolerance and irritable bowel syndrome. Nutr Burbank Los Angel Cty Calif. 2000;16(7-8).

97. Shepherd SJ, Gibson PR. Fructose Malabsorption and Symptoms of Irritable Bowel Syndrome: Guidelines for Effective Dietary Management. J Am Diet Assoc. 2006;106(10):1631-1639. doi:10.1016/j.jada.2006.07.010

98. Vos MB, Kimmons JE, Gillespie C, Welsh J, Blanck HM. Dietary fructose consumption among US children and adults: the Third National Health and Nutrition Examination Survey. Medscape J Med. 2008;10(7).

99. Beyer PL, Caviar EM, McCallum RW. Fructose Intake at Current Levels in the United States May Cause Gastrointestinal Distress in Normal Adults. J Am Diet Assoc. 2005;105(10):1559-1566. doi:10.1016/j.jada.2005.07.002

100. M. Ledochowski BW H Bair,, Richard. Fructose- and Sorbitol-reduced Diet Improves Mood and Gastrointestinal Disturbances in Fructose Malabsorbers. Scand J Gastroenterol Scand J Gastroenterol. 2009;35(10):1048-1052.

101. Murray K, Wilkinson-Smith V, Hoad C, et al. Differential Effects of FODMAPs (Fermentable Oligo-, Di-, Mono-Saccharides and Polyols) on Small and Large Intestinal Contents in Healthy Subjects Shown by MRI. Am J Gastroenterol. 2014;109(1):110-119. doi:10.1038/ajg.2013.386

102. Cherbut C. Inulin and oligofructose in the dietary fibre concept. Br J Nutr. 2002;87(S2):S159. doi:10.1079/BJN2002532

103. Mansueto P, Seidita A, D'Alcamo A, Carroccio A. Role of FODMAPs in Patients With Irritable Bowel Syndrome. Nutr Clin Pract. 2015;30(5):665-682. doi: $10.1177 / 0884533615569886$

104. Lenhart A., Chey W.D. A systematic review of the effects of polyols on gastrointestinal health and irritable bowel syndrome. Adv Nutr Adv Nutr. 2017;8(4):587-596.

105. Yao CK, Tan H-L, van Langenberg DR, et al. Dietary sorbitol and mannitol: food content and distinct absorption patterns between healthy individuals and patients with irritable bowel syndrome. J Hum Nutr Diet. 2014;27:263-275. doi:10.1111/jhn.12144 
106. Magge S, Lembo A. Low-FODMAP Diet for Treatment of Irritable Bowel Syndrome. Gastroenterol Hepatol. 2012;8(11):739-745.

107. Ong DK, Mitchell SB, Barrett JS, et al. Manipulation of dietary short chain carbohydrates alters the pattern of gas production and genesis of symptoms in irritable bowel syndrome: Dietary FODMAPs and IBS symptoms. J Gastroenterol Hepatol. 2010;25(8):1366-1373. doi:10.1111/j.1440-1746.2010.06370.x

108. Staudacher HM, Whelan K. The low FODMAP diet: recent advances in understanding its mechanisms and efficacy in IBS. Gut. 2017;66(8):1517-1527. doi:10.1136/gutjnl-2017-313750

109. Barrett JS, Gearry RB, Muir JG, et al. Dietary poorly absorbed, short-chain carbohydrates increase delivery of water and fermentable substrates to the proximal colon. Aliment Pharmacol Ther. January 2010. doi:10.1111/j.13652036.2010.04237.x

110. Murray K, Wilkinson-Smith V, Hoad C, et al. Differential Effects of FODMAPs (Fermentable Oligo-, Di-, Mono-Saccharides and Polyols) on Small and Large Intestinal Contents in Healthy Subjects Shown by MRI. Am J Gastroenterol. 2014;109(1):110-119. doi:10.1038/ajg.2013.386

111. Major G, Pritchard S, Murray K, et al. Colon Hypersensitivity to Distension, Rather Than Excessive Gas Production, Produces Carbohydrate-Related Symptoms in Individuals With Irritable Bowel Syndrome. Gastroenterology. 2017;152(1):124133.e2. doi:10.1053/j.gastro.2016.09.062

112. Eswaran S. Low FODMAP in 2017: Lessons learned from clinical trials and mechanistic studies. Neurogastroenterol Motil. 2017;29(4):e13055. doi: $10.1111 / \mathrm{nmo} .13055$

113. Halmos EP. When the low FODMAP diet does not work: Treatments for irritable bowel syndrome. J Gastroenterol Hepatol. 2017;32:69-72. doi:10.1111/jgh.13701

114. Halmos EP, Christophersen CT, Bird AR, Shepherd SJ, Gibson PR, Muir JG. Diets that differ in their FODMAP content alter the colonic luminal microenvironment. Gut. 2015;64(1):93-100. doi:10.1136/gutjnl-2014-307264

115. Shepherd S, Parker F, Muir J, Gibson P. Dietary Triggers of Abdominal Symptoms in Patients With Irritable Bowel Syndrome: Randomized PlaceboControlled Evidence. Clin Gastroenterol Hepatol. 2008;6(7):765-771. doi:10.1016/j.cgh.2008.02.058

116. Riggs S. The Low-FODMAP Diet: An Approach for Controlling Irritable Bowel Syndrome. Top Clin Nutr. 2014;29(4):304-312. doi:10.1097/TIN.0000000000000015 
117. Gibson PR. Use of the low-FODMAP diet in inflammatory bowel disease: Low FODMAP diet in IBD. J Gastroenterol Hepatol. 2017;32:40-42.

doi:10.1111/jgh. 13695

118. Schumann D, Klose P, Lauche R, Dobos G, Langhorst J, Cramer H. Low fermentable, oligo-, di-, mono-saccharides and polyol diet in the treatment of irritable bowel syndrome: A systematic review and meta-analysis. Nutrition. 2018;45:24-31. doi:10.1016/j.nut.2017.07.004

119. Marsh A, Eslick EM, Eslick GD. Does a diet low in FODMAPs reduce symptoms associated with functional gastrointestinal disorders? A comprehensive systematic review and meta-analysis. Eur J Nutr. 2016;55(3):897-906. doi:10.1007/s00394-0150922-1

120. Altobelli E, Del Negro V, Angeletti P, Latella G. Low-FODMAP Diet Improves Irritable Bowel Syndrome Symptoms: A Meta-Analysis. Nutrients. 2017;9(9):940. doi:10.3390/nu9090940

121. Varjú P, Farkas N, Hegyi P, et al. Low fermentable oligosaccharides, disaccharides, monosaccharides and polyols (FODMAP) diet improves symptoms in adults suffering from irritable bowel syndrome (IBS) compared to standard IBS diet: A meta-analysis of clinical studies. Stengel A, ed. PLOS ONE. 2017;12(8):e0182942. doi:10.1371/journal.pone. 0182942

122. Halmos EP, Power VA, Shepherd SJ, Gibson PR, Muir JG. A Diet Low in FODMAPs Reduces Symptoms of Irritable Bowel Syndrome. Gastroenterology. 2014;146(1):67-75.e5. doi:10.1053/j.gastro.2013.09.046

123. Staudacher HM, Lomer MCE, Anderson JL, et al. Fermentable Carbohydrate Restriction Reduces Luminal Bifidobacteria and Gastrointestinal Symptoms in Patients with Irritable Bowel Syndrome. J Nutr. 2012;142(8):1510-1518. doi:10.3945/jn.112.159285

124. Peters SL, Yao CK, Philpott H, Yelland GW, Muir JG, Gibson PR. Randomised clinical trial: the efficacy of gut-directed hypnotherapy is similar to that of the low FODMAP diet for the treatment of irritable bowel syndrome. Aliment Pharmacol Ther. 2016;44(5):447-459. doi:10.1111/apt.13706

125. Eswaran SL, Chey WD, Han-Markey T, Ball S, Jackson K. A Randomized Controlled Trial Comparing the Low FODMAP Diet vs. Modified NICE Guidelines in US Adults with IBS-D. Am J Gastroenterol. 2016;111(12):1824-1832. doi:10.1038/ajg.2016.434

126. Böhn L, Störsrud S, Liljebo T, et al. Diet Low in FODMAPs Reduces Symptoms of Irritable Bowel Syndrome as Well as Traditional Dietary Advice: A Randomized Controlled Trial. Gastroenterology. 2015;149(6):1399-1407.e2. doi:10.1053/j.gastro.2015.07.054 
127. Pedersen N, Andersen NN, Végh Z, et al. Ehealth: Low FODMAP diet vs Lactobacillus rhamnosus GG in irritable bowel syndrome. World J Gastroenterol. 2014;20(43):16215. doi:10.3748/wjg.v20.i43.16215

128. Chumpitazi BP, Cope JL, Hollister EB, et al. Randomised clinical trial: gut microbiome biomarkers are associated with clinical response to a low FODMAP diet in children with the irritable bowel syndrome. Aliment Pharmacol Ther. 2015;42(4):418-427. doi:10.1111/apt.13286

129. McIntosh K, Reed DE, Schneider T, et al. FODMAPs alter symptoms and the metabolome of patients with IBS: a randomised controlled trial. Gut. 2017;66(7):1241-1251. doi:10.1136/gutjnl-2015-311339

130. O'Keeffe M, Jansen C, Martin L, et al. Long-term impact of the low-FODMAP diet on gastrointestinal symptoms, dietary intake, patient acceptability, and healthcare utilization in irritable bowel syndrome. Neurogastroenterol Motil. 2018;30(1):e13154. doi:10.1111/nmo.13154

131. Harvie RM, Chisholm AW, Bisanz JE, et al. Long-term irritable bowel syndrome symptom control with reintroduction of selected FODMAPs. World J Gastroenterol. 2017;23(25):4632. doi:10.3748/wjg.v23.i25.4632

132. de Roest RH, Dobbs BR, Chapman BA, et al. The low FODMAP diet improves gastrointestinal symptoms in patients with irritable bowel syndrome: a prospective study. Int J Clin Pract. 2013;67(9):895-903. doi:10.1111/ijcp.12128

133. Valeur J, Smastuen MC, Knudsen T, Lied GA, Roseth AG. Microbial DNA Markers Associated with Response to a Low Fodmap Diet in Patients with Irritable Bowel Syndrome. Gastroenterology. 2017;152(5):44.

134. Staudacher HM, Lomer MC., Farquharson FM, et al. A Diet Low in FODMAPs Reduces Symptoms in Patients With Irritable Bowel Syndrome and A Probiotic Restores Bifidobacterium Species: A Randomized Controlled Trial. YGAST Gastroenterol. 2017;153(4).

135. Scott KP, Antoine J-M, Midtvedt T, van Hemert S. Manipulating the gut microbiota to maintain health and treat disease. Microb Ecol Health Dis. 2015;26(0). doi:10.3402/mehd.v26.25877

136. Vincenzi M, Del Ciondolo I, Pasquini E, Gennai K, Paolini B. Effects of a Low FODMAP Diet and Specific Carbohydrate Diet on Symptoms and Nutritional Adequacy of Patients with Irritable Bowel Syndrome: Preliminary Results of a Single-blinded Randomized Trial. J Transl Intern Med. 2017;5(2):120-126.

137. Staudacher H, Ross F, Briscoe Z, Irving P, Whelan K, Lomer M. PTU-183 Advice from a dietitian regarding the low fodmap diet broadly maintains nutrient 
intake and does not alter fibre intake. Gut. 2015;64(Suppl 1):A143.2-A144. doi:10.1136/gutjnl-2015-309861.298

138. Yang YJ, Kim MK, Hwang SH, Ahn Y, Shim JE, Kim DH. Relative validities of 3-day food records and the food frequency questionnaire. Nutr Res Pract. 2010;4(2):142. doi:10.4162/nrp.2010.4.2.142

139. Zia JK, Chung C-F, Schroeder J, et al. The feasibility, usability, and clinical utility of traditional paper food and symptom journals for patients with irritable bowel syndrome. Neurogastroenterol Motil. 2017;29(2):e12935. doi:10.1111/nmo.12935

140. National Cancer Institute. Food Frequency Questionnaire at a Glance. https://dietassessmentprimer.cancer.gov/profiles/questionnaire/. Accessed January 3, 2018.

141. National Cancer Institute. 24-Hour Dietary Recall (24HR) At a Glance. https://dietassessmentprimer.cancer.gov/profiles/recall/. Accessed January 3, 2018.

142. National Cancer Institute. Food Record at a Glance. https://dietassessmentprimer.cancer.gov/profiles/record/. Accessed August 24, 2017.

143. Whigham L, Joyce T, Harper G, et al. Clinical effectiveness and economic costs of group versus one-to-one education for short-chain fermentable carbohydrate restriction (low FODMAP diet) in the management of irritable bowel syndrome. $J$ Hum Nutr Diet. 2015;28(6):687-696. doi:10.1111/jhn.12318

144. National Academy of Sciences. Dietary Reference Intakes (DRIs): Acceptable Macronutrient Distribution Ranges. https://www.ncbi.nlm.nih.gov/books/NBK56068/table/summarytables.t5/?report=obj ectonly. Accessed February 19, 2018.

145. US Department of Health and Human Services, US Department of Agriculture. Dietary Guidelines for Americans 2015-2020. https://health.gov/dietaryguidelines/2015/resources/20152020_Dietary_Guidelines.pdf. Accessed February 15, 2018.

146. United States Department of Agriculture, Center for Nutrition Policy and Promotion. The Food Supply and Dietary Fiber: Its Availability and Effect on Health. https://www.cnpp.usda.gov/sites/default/files/nutrition_insights_uploads/Insight36.pd f. Accessed February 12, 2018.

147. Böhn L, Störsrud S, Simrén M. Nutrient intake in patients with irritable bowel syndrome compared with the general population: Nutrient intake in IBS. Neurogastroenterol Motil. 2013;25(1):23-e1. doi:10.1111/nmo.12001 
148. Williams EA, Nai X, Corfe BM. Dietary intakes in people with irritable bowel syndrome. BMC Gastroenterol. 2011;11:9.

149. US Department of Health and Human Services, US Department of Agriculture. Scientific Report of the 2015 Dietary Guidelines Advisory Committee.

https://health.gov/dietaryguidelines/2015-scientific-report/PDFs/Scientific-Report-ofthe-2015-Dietary-Guidelines-Advisory-Committee.pdf. Accessed February 15, 2018.

150. Fulgoni VL, Keast DR, Bailey RL, Dwyer J. Foods, Fortificants, and Supplements: Where Do Americans Get Their Nutrients? J Nutr. 2011;141(10):18471854. doi:10.3945/jn.111.142257

151. Staudacher HM, Kurien M, Whelan K. Nutritional implications of dietary interventions for managing gastrointestinal disorders: Curr Opin Gastroenterol. December 2017:1. doi:10.1097/MOG.0000000000000421

152. Ortega RM. Dietary assessment methods: dietary records. Nutr Hosp. 2015;(3):38-45. doi:10.3305/nh.2015.31.sup3.8749

153. Monash University. CNAQ. http://www.cnaq.com.au/\#. Accessed March 1, 2018.

154. Barrett JS, Gibson PR. Development and validation of a comprehensive semiquantitative food frequency questionnaire that includes FODMAP intake and glycemic index. J Am Diet Assoc. 2010;110(10):1469-1476.

155. Patrick DL, Drossman DA, Frederick IO, Dicesare J, Puder KL. Quality of Life in Persons with Irritable Bowel Syndrome (Development and Validation of a New Measure). Dig Sci Dig Dis Sci. 1998;43(2):400-411.

156. Bijkerk CJ, de Wit NJ, Muris JW, Jones RH, Knottnerus JA, Hoes AW. Outcome measures in irritable bowel syndrome: comparison of psychometric and methodological characteristics. Am J Gastroenterol. 2003;98(1):122-127. 


\section{Appendix A: Class Outline}

Lesson Topic: The Low-FODMAP Diet

Lesson Overview: The intent of this lesson is to introduce the FODMAP diet to individuals with IBS. Subjects will be educated about its concept, and instructed on its application.

Lesson Objectives: By the end of this lesson, participants will be able to:

1. Explain the concept of the low FODMAP diet.

2. Identify high and low FODMAP foods.

3. Evaluated products and menus based on their FODMAP content.

4. Modify meals to contain low FODMAP options.

\section{Materials Needed:}

1. Handouts

2. Lesson PowerPoint

3. Class Evaluation 


\section{Post-Lesson Packet}

\section{Lesson Outline:}

1. Distribute binder with materials.

2. Introduction.

Good evening everyone! My name is Julie Richards, and I'm a graduate student in the Medical Dietetics Program at The Ohio State University.

\section{Introduce Research Study}

We are so pleased that you have chosen to attend our IBS Nutrition Education class. An important part of our work with Irritable Bowel Syndrome is our research that we are conducting regarding the nutrition therapy for IBS. We hope that you will consider being part of our research group. After the education session, we will be discussing the research and we hope that you will stay to learn more about it.

\section{Begin Explaining IBS}

You are all here today because you were referred to the nutrition department for dietary advice regarding a diagnosis, or suspected underlying issue, of irritable bowel syndrome, or IBS.

Your primary care providers may have already gone over some information about IBS with you, so we won't spend too much time on that today. Briefly, IBS is a functional 
disorder. This means that those affected with the disorder experience unpleasant symptoms without having any abnormal occurrences or changes within the structure of the body. These symptoms may include nausea, bloating, abdominal pain or discomfort, fatigue, diarrhea, constipation, or a mixture of both.

ASK: Can any of you relate to these symptoms?

Researchers are still trying to understand the mechanisms behind the disease. It appears to be very complex, and we still don't know exactly what causes it, which is why current treatments focus on controlling symptoms rather than providing a cure. There have been several theories about the cause, however, and one of the theories is that the intestines (or bowels) of individuals with IBS are hypersensitive. This means that they have a stronger sense or reaction to certain ingested foods than people who don't have the disorder experience. There are foods that everyone has difficulty digesting, but for individuals with IBS they are more sensitive to this process. There are also changes in motility experienced by IBS patients, causing IBS patients to feel pain when a person without IBS would not normally.

In the past several years, diets that exclude "irritating" food items have been suggested for individuals with IBS.

ASK: Has anyone tried following a specific diet or restricting certain foods for their IBS? 
For years, we have been trying to establish a concrete nutritional treatment for IBS. Today we will be discussing a diet that research has consistently demonstrated to be effective in reducing IBS symptoms. In most studies, up to $75 \%$ of patients show satisfying symptom relief and improvement in quality of life by following the diet, which is an impressive statistic! It is called the low FODMAP diet, and it was first developed in Monash University in Australia.

I want to talk a little about what this class will be setup like. This class will be divided into a lecture section, an activity, and a Q\&A section about the diet. Please feel free to write down any questions you come up with as we go through the lecture section, and we will provide plenty of time after the lecture for all questions to be answered.

Our objectives for the rest of the class are: to explain the concept of the low FODMAP diet, to identify high and low FODMAP foods, to evaluate products and menus based on FODMAP content, and to modify meals and snacks to contain low FODMAP options.

As I previously stated, the low FODMAP diet was first developed by scientists at Monash University in Australia. We will start with a video they have put together to help explain how foods containing FODMAPs cause IBS symptoms. We will go into detail about the ideas explained after the video, but sometimes it is helpful to have a visual!

FODMAP is an acronym for fermentable oligosaccharides, disaccharides, monosaccharides, and polyols. It basically stands for a group of sugars, otherwise known 
as carbohydrates, that are known for being poorly digested and absorbed in humans. Typically, carbohydrates are broken down and absorbed in our small intestines. However, the carbohydrates called FODMAPs do not follow this pattern because there is an inability to completely digest these foods in the small intestine.

When carbohydrates are not broken down and absorbed properly, they continue through the GI tract to the large intestine. The large intestine does not have the same absorptive abilities of the small intestine, because carbohydrates are not expected to flow there in high quantities. As they continue to the large intestine, they draw excess water into the intestine, which can result in diarrhea. In addition, the bacteria naturally found in the large intestine will consume the carbohydrates for energy in a process called fermentation. Because of fermentation, gas is released, which distends (or expands) the intestines, resulting in pain or discomfort. Having to pass gas is also a frequent consequence of fermentation. Everyone who consumes FODMAPs experience this process. However, not everyone will experience the symptoms of the process, because people with IBS have the hypersensitivity that we discussed earlier, which exaggerate the responses to the FODMAPs.

\section{Introduce Low-FODMAP Diet}

So now that we know why FODMAPs increases symptoms of IBS, it is important to talk about which foods are high in FODMAPs, and how to adopt a low FODMAP diet to reduce these symptoms. 
The individual types of carbohydrates that are known to be FODMAPs are: fructose, lactose, fructans, galactans, and polyols. They are found in many fruits, vegetables, and whole grains, as well as dairy products.

There is a wide variation in how individuals with IBS react to these FODMAPs. Some people are sensitive to only one or two types of FODMAPs, while others do not tolerate any of them. Sensitivity is also dose dependent, so eating a large amount of FODMAPs in one sitting can also cause symptoms. Therefore, the first phase of the low FODMAP diet is based on restricting your intake of all the foods considered high in FODMAPs and replacing them with foods lower in FODMAPs for a specified period. Research has shown different lengths of time that the elimination phase is necessary, but it would be somewhere between 2-8 weeks typically. After the elimination phase, there will be a reintroduction phase, where FODMAPs are reintroduced a group at a time to test individual tolerance.

The first phase of the low FODMAP diet is based on restricting your intake of all the foods considered high in FODMAPs and replacing them with foods lower in FODMAPs. Although there are a lot of high FODMAP foods, there are even more low FODMAP options, so the diet is definitely achievable!

6. Review high-FODMAP food list in detail.

7. Review low-FODMAP grocery list 
I want to note that some foods, such as apples, are found in more than one section because they contain more than one FODMAP. You'll see that there are no meats or oils on this list, so that means that you can still enjoy meats and oils as you would normally. Once you identify the high FODMAP foods that you typically consume, replacing those foods with similar foods that are low in FODMAPs becomes a crucial step of the diet. As you can see, many common foods that many of you may eat every day are high in FODMAPs, including garlic, onions, tomato paste, and bread. If you stop eating all these foods without properly replacing them, your diet will quickly become nutritionally inadequate. This puts you at greater risk for becoming deficient in energy, vitamins, and minerals, and we don't want that!

\section{Combinations}

Patients with IBS are not only bothered by the type of FODMAP in a meal, but also how much they consume of these FODMAPs in one setting. FODMAPs have an additive effect, which means that the greater amount you consume, the more likely you will induce symptoms. Most people, even those with IBS, can tolerate a certain quantity of FODMAPs, but once they pass that threshold, they experience symptoms. So, how can you avoid this? If you look at your low-FODMAP grocery list, you will see that moderate FODMAP choices have a serving size beside them. When you prepare or purchase your meal, avoid combining more than one "moderate" FODMAP food. Also, avoid having more than one serving of fruit per meal.

9. Label Reading 
Now we'll talk about reading food labels. You may find you want to purchase a packaged item, but you aren't sure whether it is ok on the diet. Take a look at the ingredient list. You now know the fruits, vegetables, and grains that are high in FODMAPs, but what about other ingredients? In your folder, you'll find a handout to take with you when you do your shopping that has some other common ingredients that are low in FODMAPs. Those ingredients should be ok! What if you find a label that includes something high in FODMAPs? You may not automatically need to avoid it. For example, if garlic is that high FODMAP ingredient, and it is the second on the list, you should probably avoid it. The higher up on the list the item occurs, the greater amount of that ingredient there is in that food. If it is several items down on the list, it may be a small enough amount that it won't cause you trouble. However, we'd like to stress that you should try to avoid all high FODMAP foods for at least 6 weeks.

So, now we will practice replacing high FODMAP foods with low FODMAP foods in some example meals and snacks. For each meal and snack, try to point out which foods are high in FODMAPs, and suggest what low FODMAP item you might use to replace it. Remember, we want to make sure our meals are varied and balanced, so try replacing fruits with fruits, vegetables with vegetables, and grains with grains.

10. Review two example food labels.

11. Meal replacement practice.

For our sample breakfast, we have a bowl of wheat bran cereal with $1 \%$ milk and a banana. 
ASK: Which parts of this breakfast are high in FODMAPs?

The wheat bran would be high in fructans and galactans, and the $1 \%$ milk would be high in lactose, all high in FODMAPs.

ASK: What low FODMAP food might you replace those items with?

One example that we came up with would be replacing the wheat bran with either oatmeal or an cereal made from oats like Cheerios, because oats are low in FODMAPs, and using almond milk in place of $1 \%$ milk.

For a morning snack, we might try peanut butter with some apple slices.

ASK: Is anything in this snack high in FODMAPs?

Apples are high in fructose and polyols, so it is a good fruit to avoid.

ASK: What fruit might you replace the apple with?

Any fruit that is low in FODMAPs, like strawberries, would be an appropriate replacement!

For lunch we will try a turkey sandwich on whole wheat bread with lettuce, Swiss cheese, and mustard; a side salad made with cauliflower, broccoli, and carrots; and an orange. ASK: Which components of our lunch should we change?

The whole wheat bread in our sandwich is high in fructans and galactans, and the cauliflower is high in polyols.

ASK: What would you replace these food items with? 
Any bread made without grains that are high in FODMAPs would suffice, such as a corn tortilla wrap. Cauliflower could be replaced with any low FODMAP veggie like bell peppers. It is also important to evaluate the dressing used fore the side salad!

We'll have some almonds for an afternoon snack.

ASK: Is this a low or high FODMAP snack?

Almonds are low in FODMAPs! There is nothing we need to change about this snack.

Finally, for dinner we will have lemon garlic baked chicken, mashed potatoes, and asparagus.

ASK: What in this meal would induce IBS symptoms?

The garlic in our baked chicken is high in fructans and galactans, and the asparagus in high in fructose.

ASK: How might you change this dinner?

One good thing to note for people who love garlic is that it is the actually plant that causes the trouble. You can buy or make garlic-infused oil, and as long as the garlic is removed from the oil before consuming it, this shouldn't cause any symptoms. You could also use other herbs to season the chicken. You can replace the asparagus with any low FODMAP veggie, like green beans. It is also important to evaluate how the mashed potatoes are being made - make sure they don't have milk or garlic added to them!

Section 4 of your binder is "how to make a low-FODMAP meal" if you would like to refer to that during this slide. Have you all ever seen MyPlate? It was created by the 
USDA to help people adopt a healthier lifestyle. So as you can see, the low FODMAP diet does require some extra thought and planning, and may require you to avoid some fruits, vegetables, and whole grains that you like, but it is not too challenging to find replacements! In addition, our meal examples resemble the healthy plate recommendations, where we are encouraged to have 3-4 food groups represented per meal, and 1-2 food groups per snack. It is important to strive for this pattern in as many meals and snacks as you can to ensure that you are eating a balanced diet, with a good variety of carbohydrates, proteins, healthy fats, vitamins, and minerals.

One example of a meal you could make that would include all the different groups would be stir fry (gives examples of what could be put in it using "how to make a lowFODMAP meal sheet").

12. Special occasions when on the low-FODMAP diet Let's say you have a special occasion coming up, or you want to spend time with friends or family at your favorite restaurant. You can definitely do that on a low FODMAP diet! We'll give you a few tips for how to do that!

With parties and special occasions, just ask whoever made a food what they put in it. Have your food lists with you or keep pictures of them on your phone so you can be confident you're making a good food choice!

Many restaurants now display their menus online. If you know where you are planning to eat, look at the menu online and find a few dishes that will suit the diet. If you aren't sure, 
you can try calling a restaurant during a non-peak hour (many restaurants are pretty slow after they first open or sometime after the lunch rush and before dinnertime), and chefs may be able to help you out with any questions.

Another helpful tip is to look for restaurants that offer gluten-free items or menus. We want to stress this-you do NOT have to follow a gluten-free diet. However, gluten-free grains like rice and quinoa are naturally low in FODMAPs. Since gluten-free foods are becoming more and more available, this can be helpful for you when you go out to eat! We have included in your folders a list of some Columbus restaurants that offer lots of gluten-free items.

\section{Review of recommended online resources}

Here are some online resources that you can refer to for further guidance on the lowFODMAP diet. Kate Scarlata is who has made a lot of our teaching material in your binder, and she is one of the leading dietitians for the low-FODMAP diet. Her blog would be a great resource for you. Monash University is the university that created the low-FODMAP diet, and it would be a great resource to refer to as well. There is an app created by Monash University.

14. Explanation of research study 


\section{Appendix B: Demographics and Health Questionnaire}

\section{Demographics and Health Questionnaire}

The following questionnaire will help us evaluate your health status. Please fill out and mail back to us in the stamped envelope we provided or bring it with you on day of your class session.

If you have any questions, please contact: Marcia Nahikian-Nelms, PhD, RDN 614292-4758.

1. Name:

2. Gender: M F Other

3. Age:

4. Weight:

5. Height:

6. When were you diagnosed with IBS?:

7. Have you previously received information about nutrition therapy for your IBS from a health care provider or met with a Registered Dietitian to discuss diet for managing your IBS symptoms?

Yes No

If yes, please indicate the type of nutrition recommendation made for you: 
8. Do you currently follow a special diet to help with your IBS symptoms? Yes No

If yes, please specify or describe the diet:

9. Do you currently exclude any foods to help with your IBS symptoms? Yes No

If yes, please indicate which foods you avoid:

10. Do you engage in physical activity? Yes No

If yes, please indicate:

Type of exercise (example: walking, running, etc.):

Length of physical activity period (example: 10 minutes):

How often do you engage in such activity per week? (once, twice, etc.)

11. Do you smoke? Yes No Previously, not anymore. Year quit:

12.

12. Do you consume alcohol? Yes No

If yes, how many drinks per week on average?

13. Are you currently taking any prescription medications for your IBS? Yes No Check all that apply.

Anti cholinergic and antispasmodics: hyoscyamine (Levsin), Bentyl (dicylomine) colesevelam (Welchol)

Antidepressants:

fluoxetine(Prozac, Serafem)
Bile acid binders: Cholestyramine colestipol (Colestid)

Alosetron (Lotronex) 
imipramine (Tofranil)

Lubiprostone (Amitiza) nortriptyline (Pamelor) paroxetine (Paxil)

Others, please specify:

14. Do you take any of the following non-prescription medications to improve your IBS symptoms?

If yes, please state the name and how often you take these medications:

\begin{tabular}{|l|l|l|l|}
\hline Type of Medication & Yes or No & $\begin{array}{l}\text { Name of } \\
\text { Medication }\end{array}$ & Frequency \\
\hline Laxative & & & \\
\hline Stool Softener & & & \\
\hline Anti-diarrheal & & & \\
\hline Gas Relief & & & \\
\hline Pain Relief & & & \\
\hline Anti-Spasmodic & & & \\
\hline
\end{tabular}

15. Do you take any of the following supplements?

If yes, please state the name and how often you take these supplements:

\begin{tabular}{|l|l|l|l|}
\hline Type of Supplement & Yes or No & $\begin{array}{l}\text { Name of } \\
\text { Supplement }\end{array}$ & Frequency \\
\hline Vitamin or Mineral & & & \\
\hline Fiber & & & \\
\hline $\begin{array}{l}\text { Probiotics or } \\
\text { Prebiotics }\end{array}$ & & & \\
\hline Other & & & \\
\hline
\end{tabular}

16. Do you take any herbal supplements/teas/oils or juices to help with your IBS symptoms?

Yes No

\begin{tabular}{|l|l|l|}
\hline $\begin{array}{c}\text { Type of } \\
\text { Supplement }\end{array}$ & Yes or No & Frequency \\
\hline Ginger & & \\
\hline Turmeric & & \\
\hline Senna & & \\
\hline Fennel seed & & \\
\hline Evening primrose oil & & \\
\hline Peppermint oil & & \\
\hline Castor oil & & \\
\hline
\end{tabular}




\begin{tabular}{|l|l|l|}
\hline Aloe & & \\
\hline
\end{tabular}

Other (please specify)

17. Do you use any alternative therapies to improve your IBS symptoms? Yes No

\begin{tabular}{|l|l|l|}
\hline \multicolumn{1}{|c|}{ Type of } & Yes or No & Frequency per week \\
\hline $\begin{array}{l}\text { Therapy } \\
\text { Cognitive behavioral }\end{array}$ & & \\
\hline Yoga & & \\
\hline Massage therapy & & \\
\hline $\begin{array}{l}\text { Hypnotherapy } \\
\text { (hypnosis) }\end{array}$ & & \\
\hline Acupuncture & & \\
\hline
\end{tabular}

Other (please specify) 
Appendix C: IBS-Symptom Severity Score (IBS-SSS) and Bristol Stool Form Scale

\section{IBS Severity Score}

Place an $\mathrm{X}$ anywhere on the line between 0 and 100 to indicate as accurately as possible the severity of your symptoms

1. How severe is your abdominal pain?

0

100

No pain Not very severe Quite severe $\quad$ Severe $\quad$ Very severe

2. Please enter the number of days that you get this abdominal pain over a 10 day period.

Number of days with abdominal pain:

$\mathrm{x} 100$

3. If you currently have abdominal distention, how severe is it?

0

100

$\begin{array}{lllll}\text { No distention } & \text { Not very } & \text { severe } & \text { Quit severe } & \text { Severe }\end{array}$ severe 


\title{
4. How satisfied are you with your bowel habits?
}

0

100

Very happy

Quite happy

Unhappy

Very unhappy

5. How much does your IBS affect or interfere with your life in general?

0

1 o

Not at all

Not much

Quite a lot

Completely

6. Currently, how often do you pass a bowel action? (please check one box)

\author{
Once a week \\ Once every 4-6 days \\ Once every 2-3 days \\ Once a day \\ 2-3 times a day \\ 4-6 times a day \\ 7 or more times a day
}

7. Please check the box that best describes your current stool: 


\section{Bristol Stool Chart}

\begin{tabular}{|l|l|}
\hline Type I & $\begin{array}{l}\text { Separate hard lumps, like nuts } \\
\text { (hard to pass) }\end{array}$ \\
\hline Type 2 & $\begin{array}{l}\text { Like a sausage but with cracks on } \\
\text { its surface }\end{array}$ \\
\hline Type 3 & $\begin{array}{l}\text { Like a sausage or snake, smooth } \\
\text { and soft }\end{array}$ \\
\hline Type 4 & $\begin{array}{l}\text { Soft blobs with clear-cut edges } \\
\text { (passed easily) }\end{array}$ \\
\hline Type 6 & $\begin{array}{l}\text { Fluffy pieces with ragged edges, a } \\
\text { mushy stool }\end{array}$ \\
\hline Type 7 & Watery, no solid pieces. \\
\hline
\end{tabular}


Appendix D: Three-Day Diet Record 


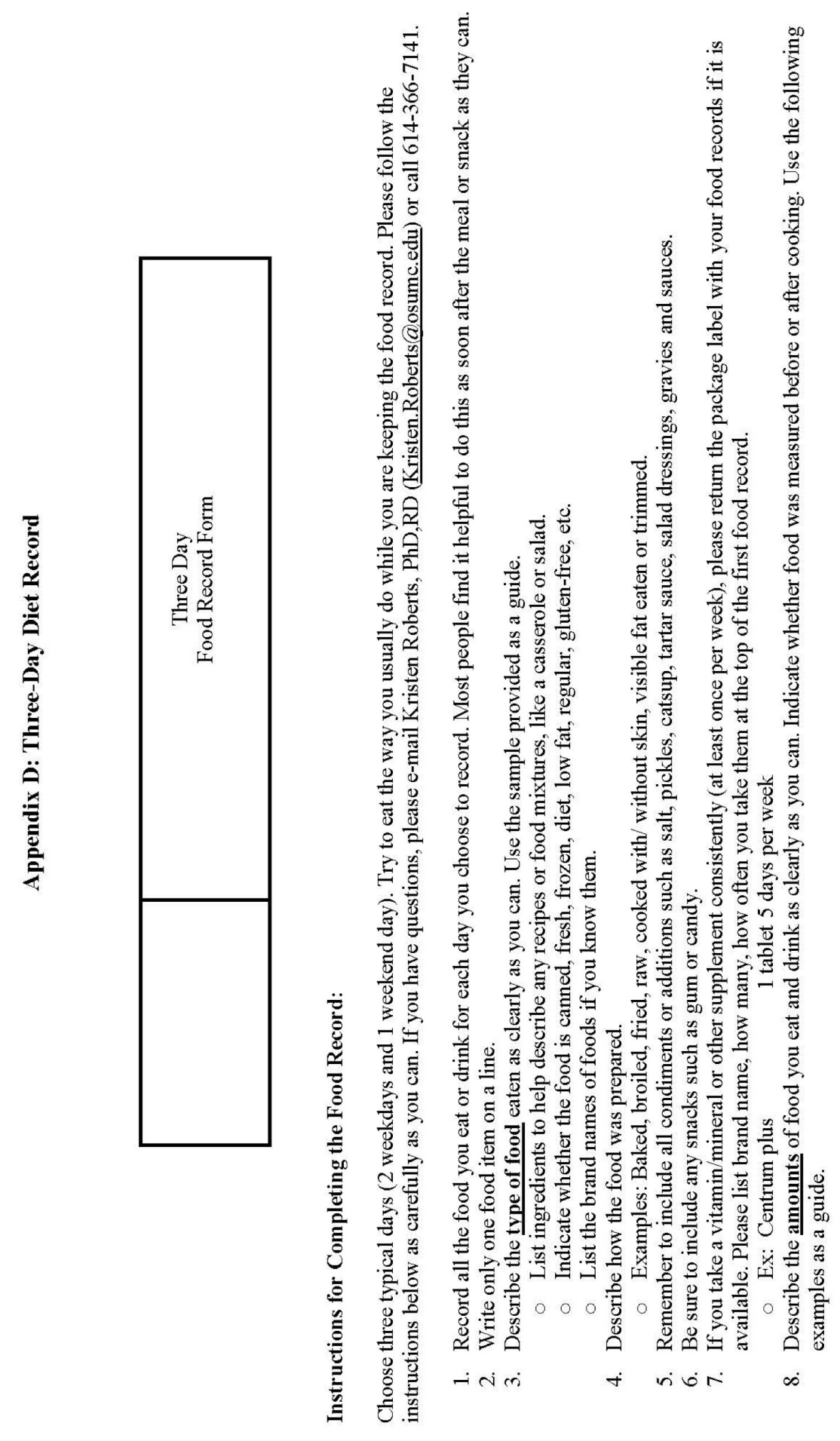




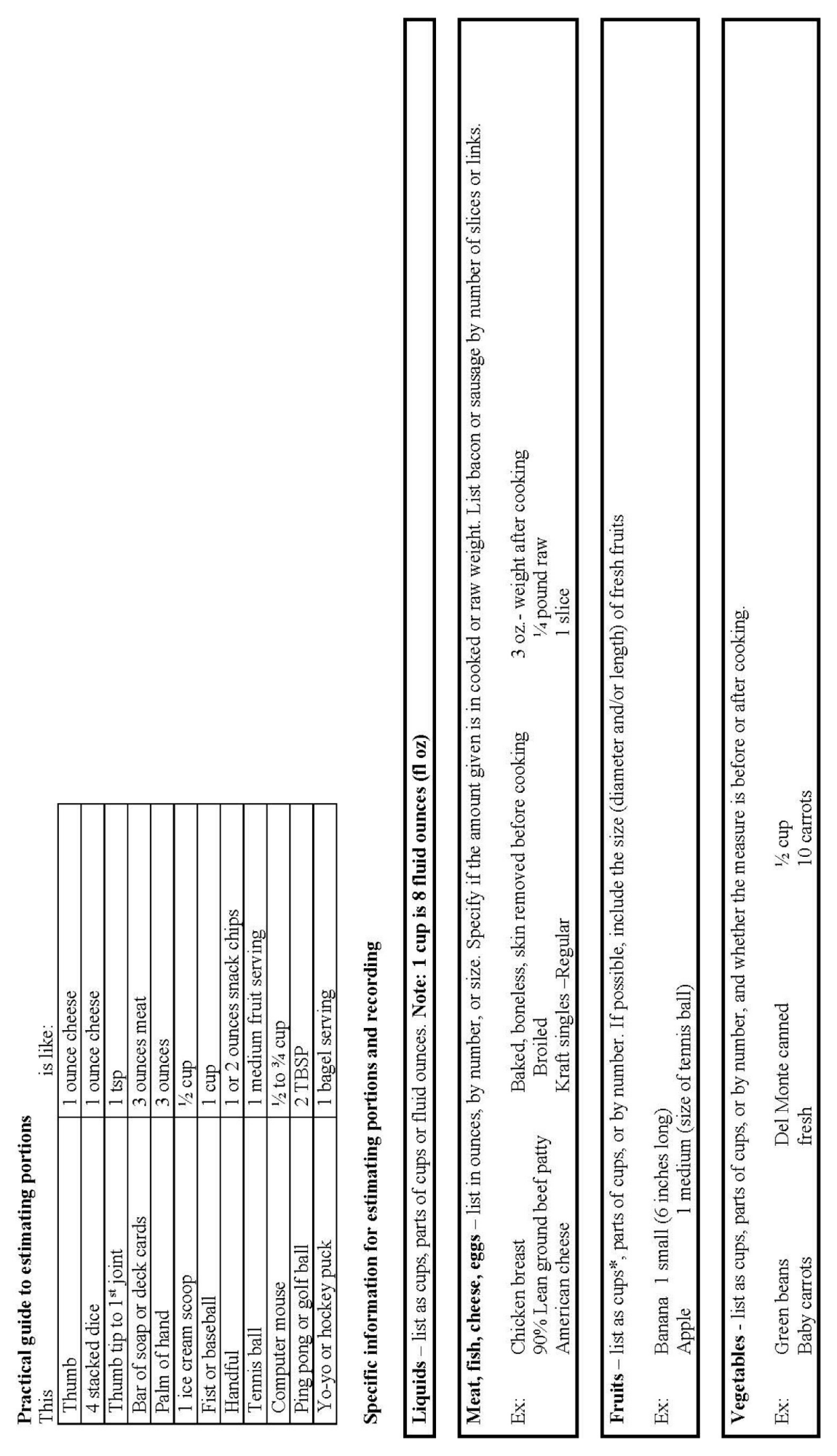




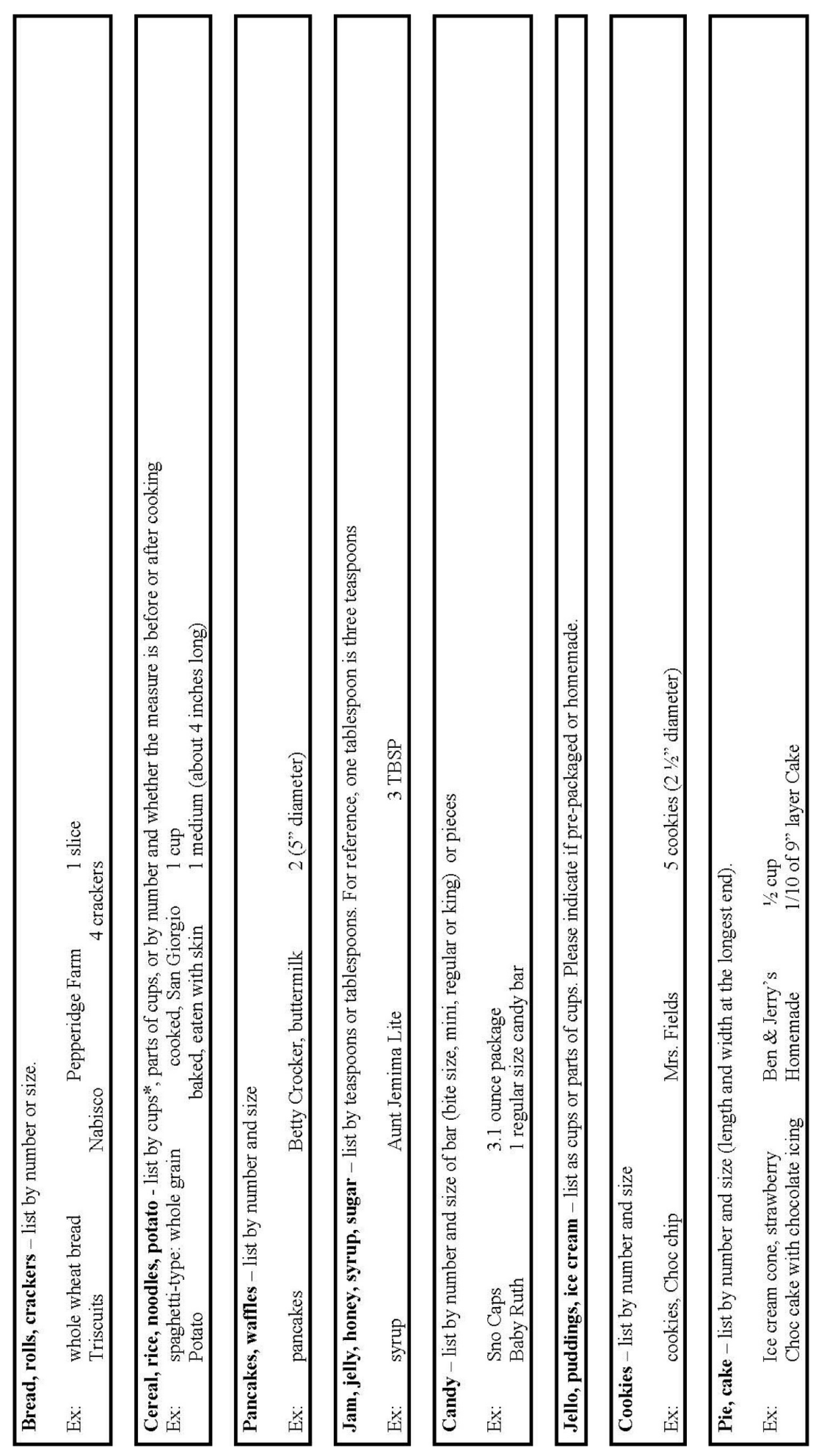



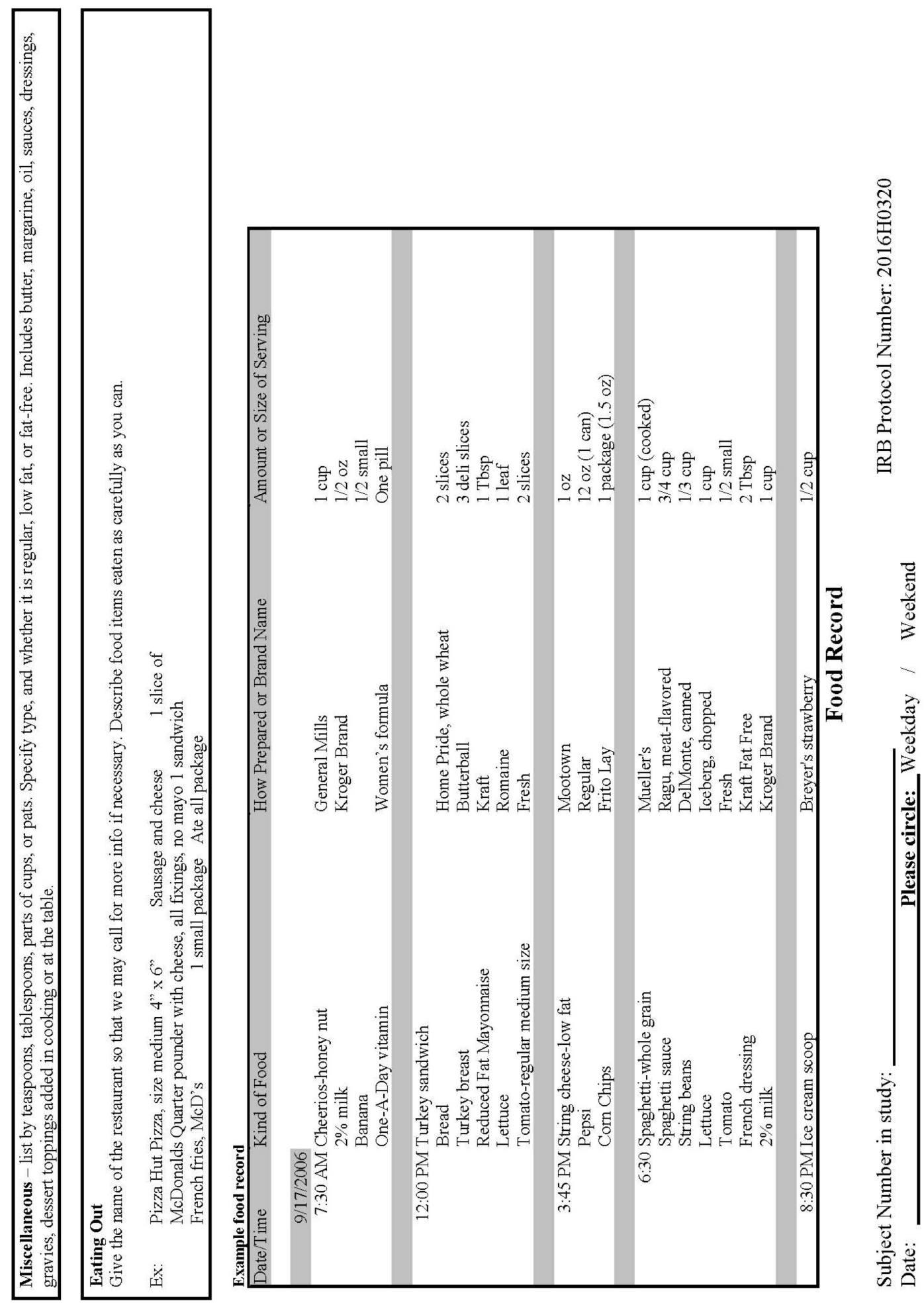


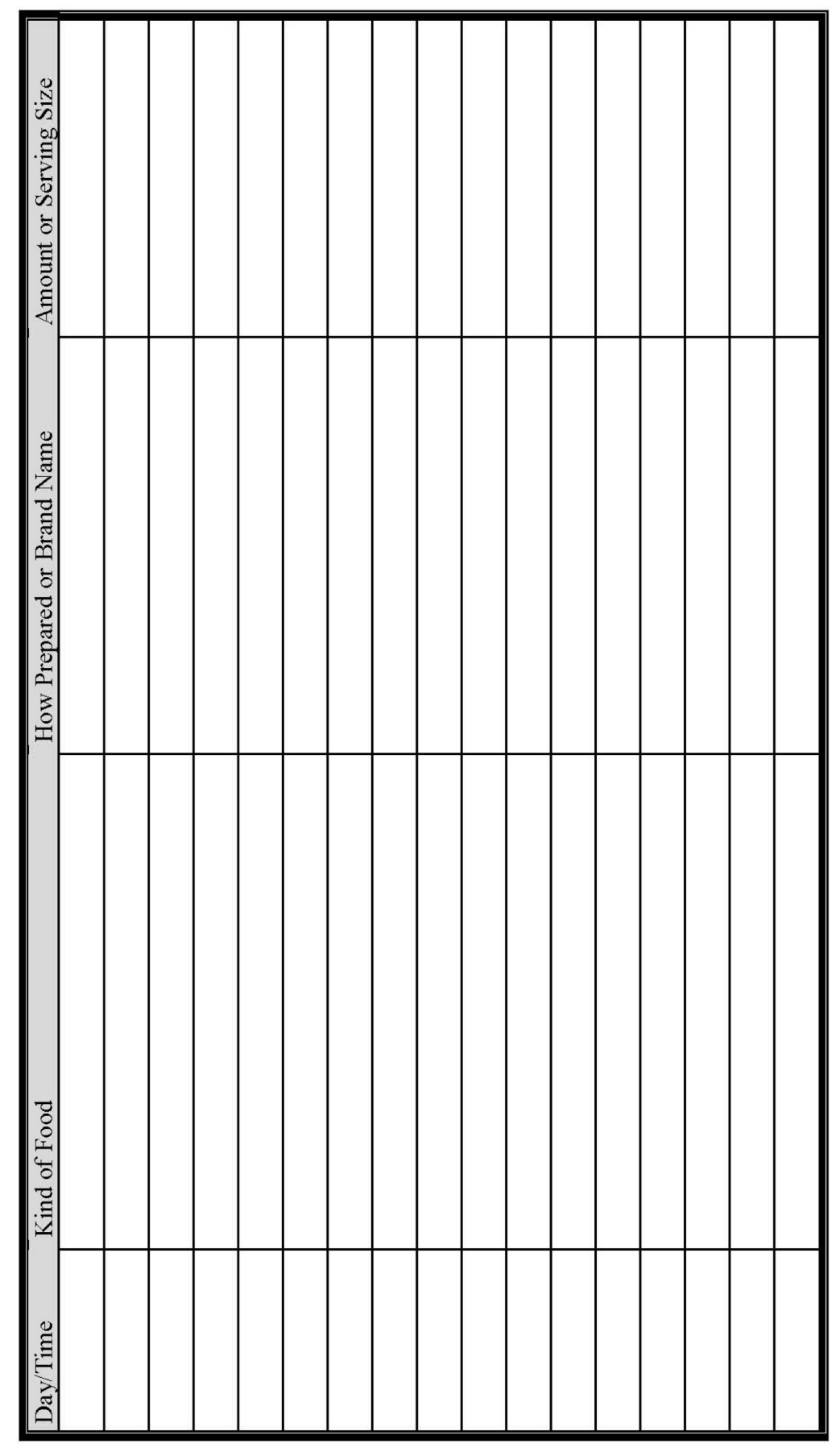




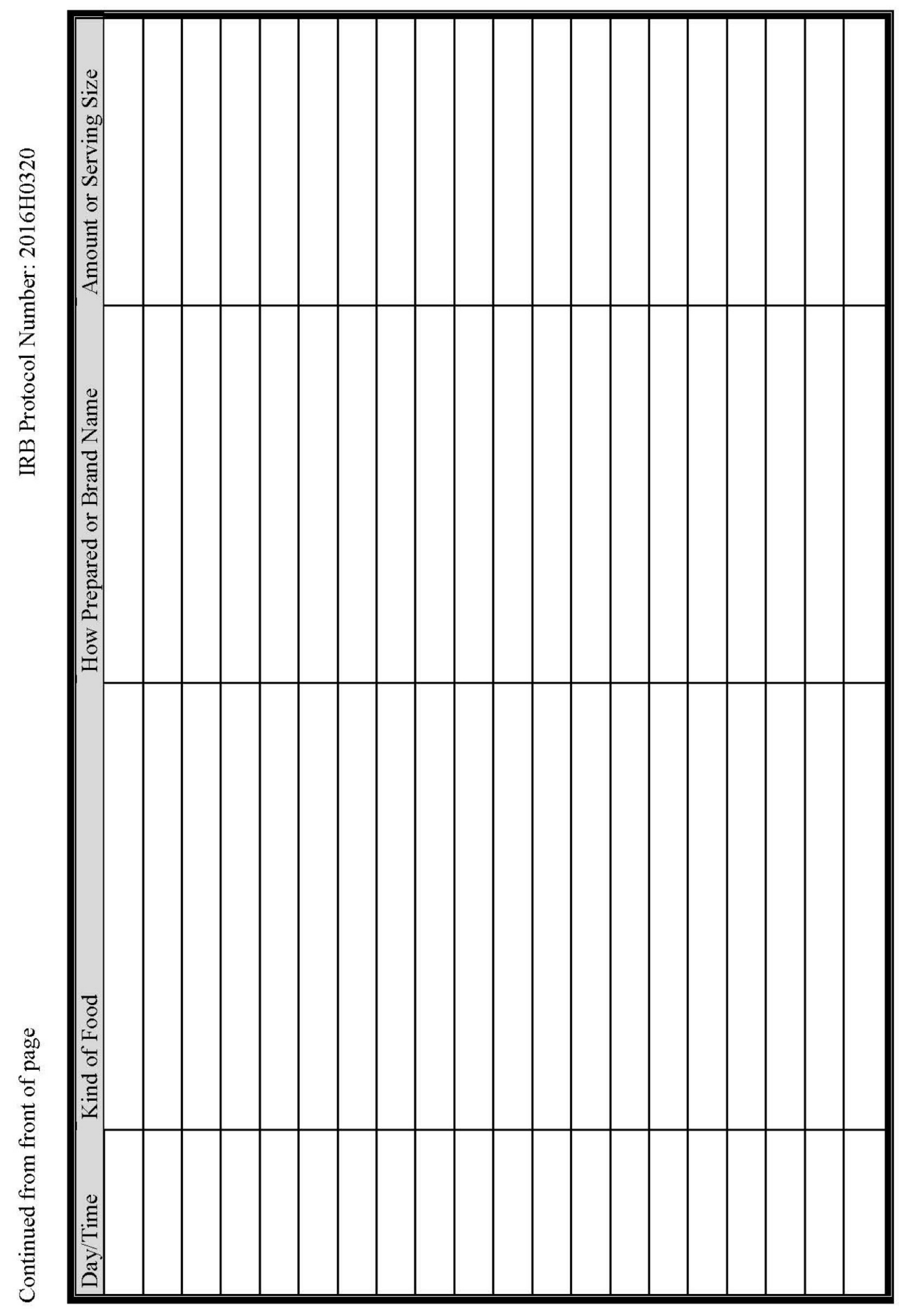




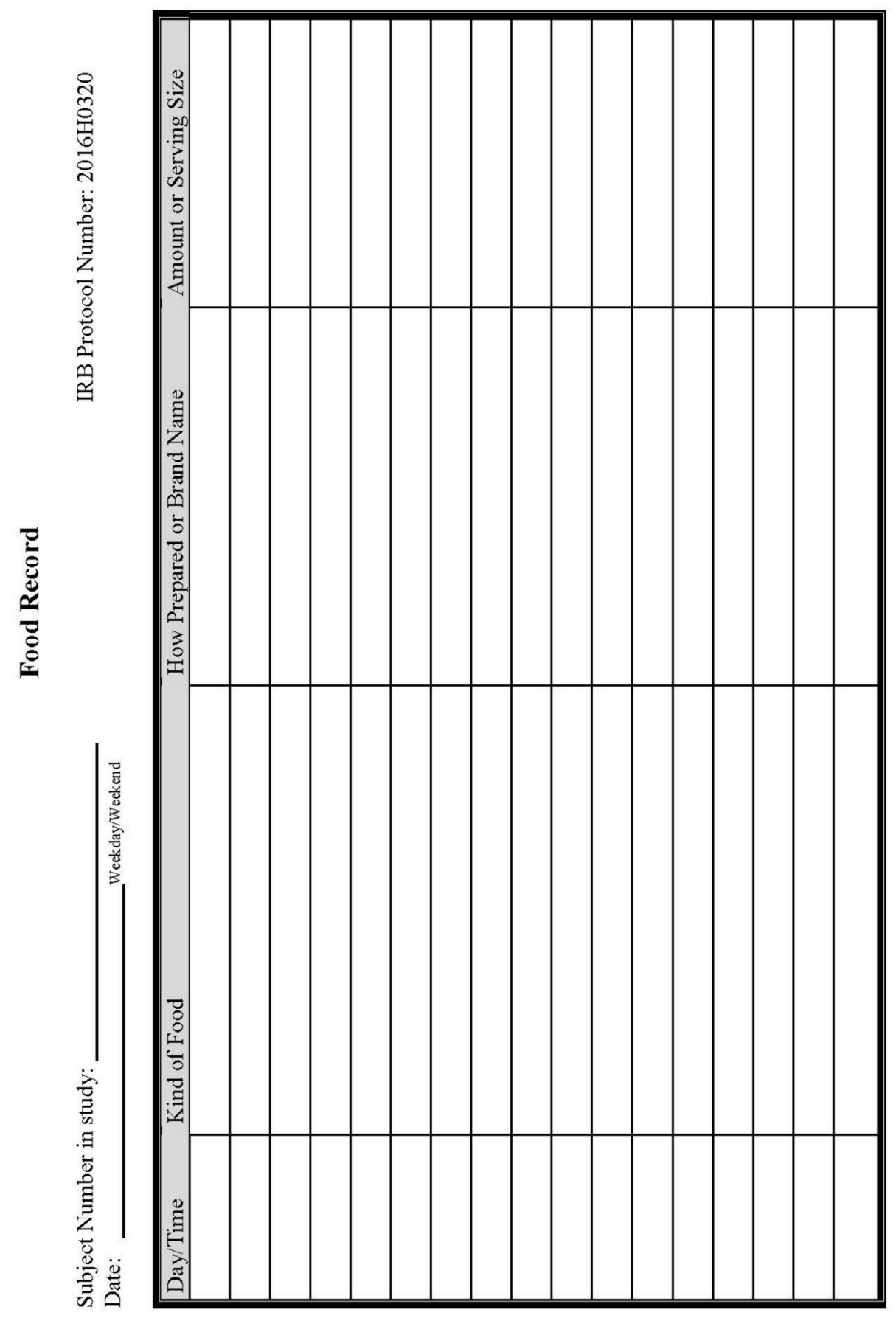




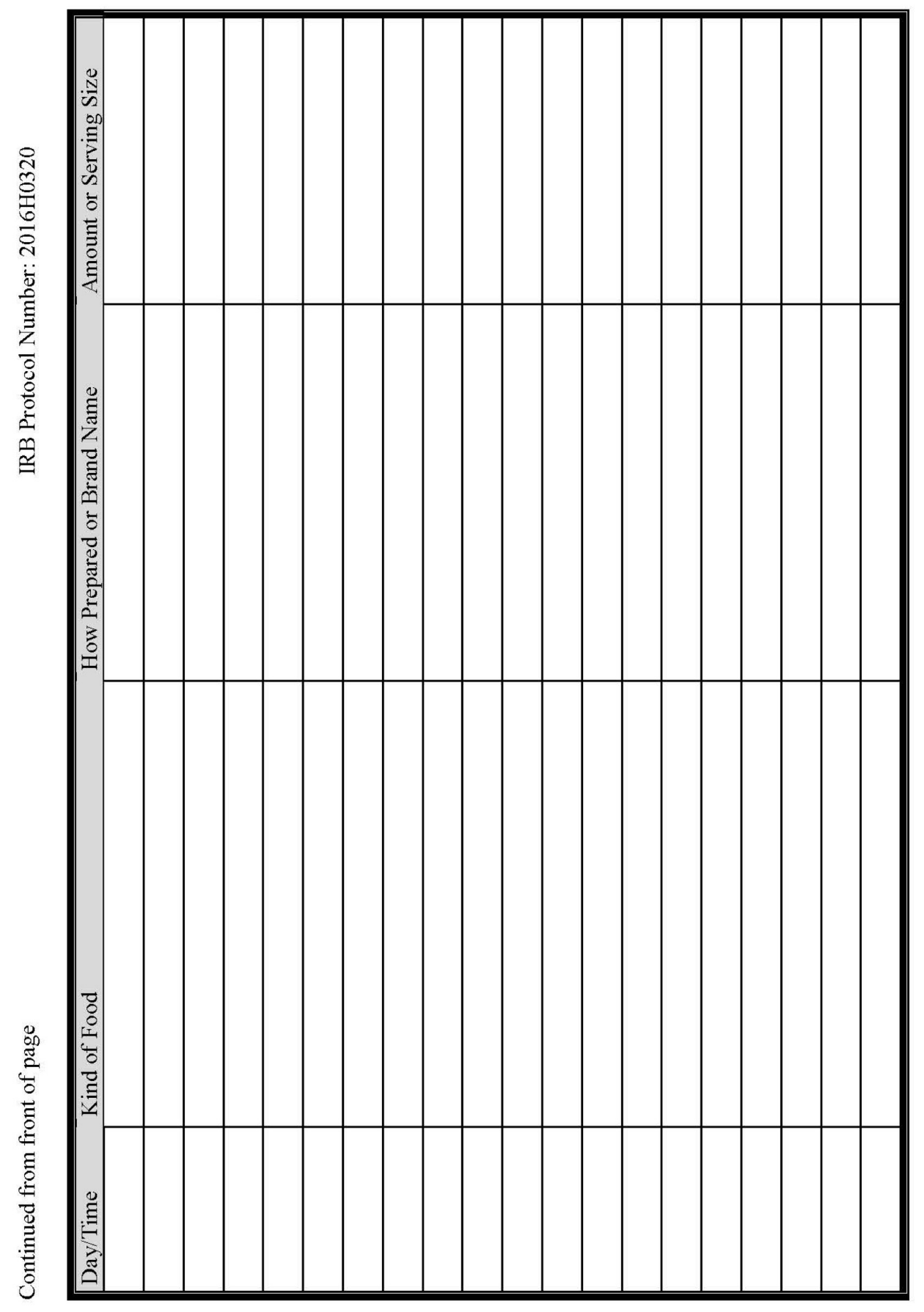




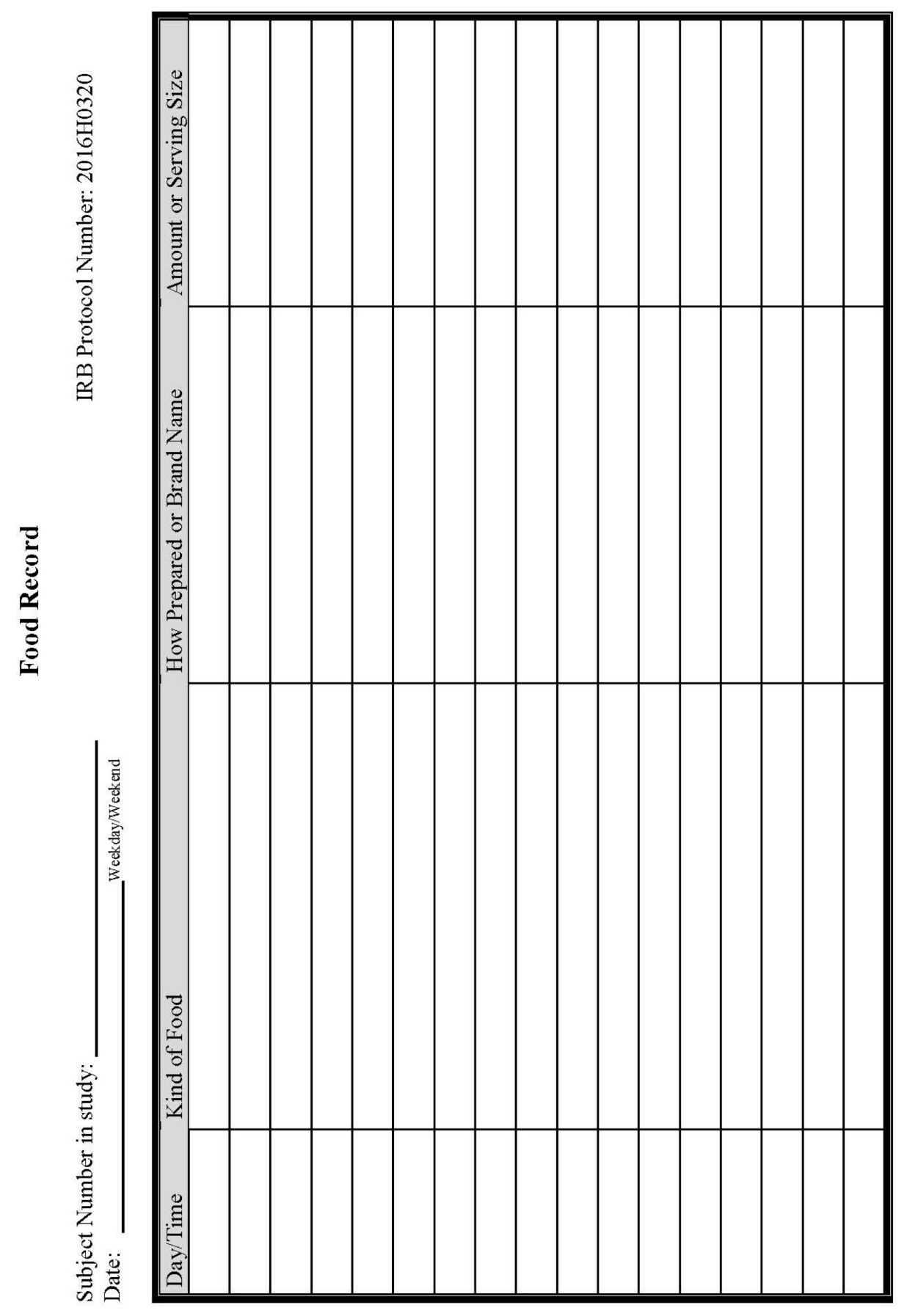




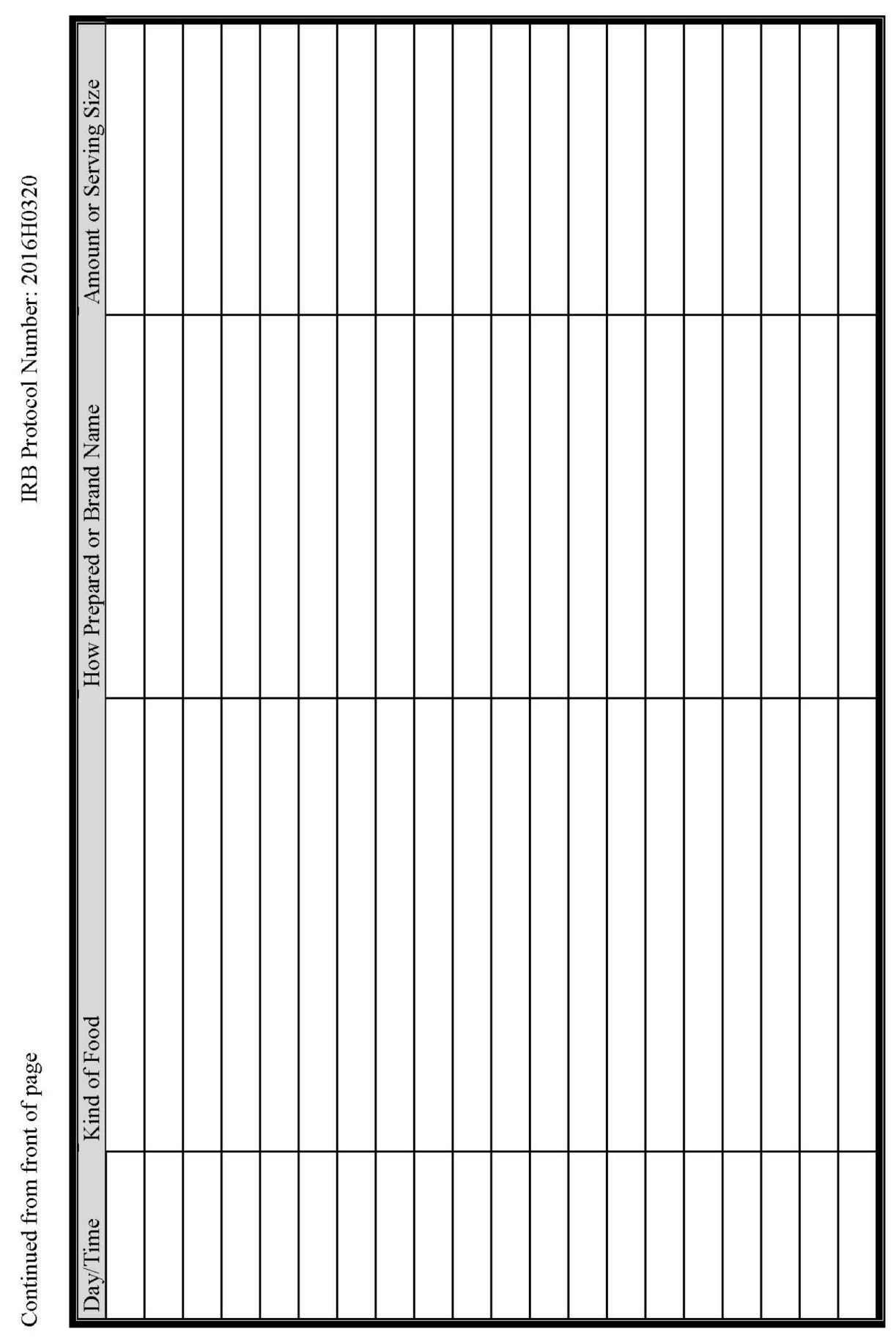




\section{Appendix E: High-FODMAP Checklist}

IRB Protocol Number: 2016H0320

Date:

\section{High FODMAP Checklist}

If you consume a high FODMAP food from this list, please circle the item and list the date of consumption. In the blank, please add how much of that food was consumed.

- Agave

- Apples

- Apricots

- Artichokes

- Asparagus

- Baked Beans

- Barley

- Blackberries

- Boysenberry

- Carob > 2 tsp

- Cashews

- Cauliflower

- Cherries

- Chickpeas $>1 / 4$ c canned

- Cottage Cheese

- Currants

- Custard

- Dates

- Evaporated Milk

- Figs (Fresh or Dried)

- Fructo-oligosaccharides (FOS)

- Garlic or Garlic Seasoning

- Garlic Powder/Salt

- Grapefruit

- High Fructose Corn Syrup (HCFS

- Honey

- Ice Cream

- Isomalt

- Inulin

- Leeks

- Lentils

- Maltitol
- Mango

- Mannitol

- Milk

- Mushrooms

- Nectarine

- Onion

- Onion Powder/Salt

- Peaches (including White)

- Pears

- Persimmons

- Pistachios

- Plums

- Prunes

- Red Kidney Beans

- Ricotta Cheese

- Rum

- Rye

- Shallot

- Sorbitol

- Soybeans \& Soybean Products

- Snow Peas

- Sugar-Snap Peas

- Sun-Dried Tomatoes

- Sweet Corn

- Tamarillo

- Teas (Chamomille, Fennel, Oolong)

- Tomato Paste

- Watermelon

- Wheat

- Sugar Free Gum (Xylitol)

- Yogurt 


\section{Appendix F: Class Evaluation}

Evaluation of Low Fermentable Oligosaccharides, Disaccharides, Monosaccharides and Polyols (FODMAP) Diet Class

1. What do you think about the length of today's session (please circle)? too long just right too short

Comments

2. What do you think about the content of today's session (please circle)?

too basic just right too complex

Comments

3. What did you think about the balance of lecturing and group discussion (please circle)?

too much lecturing just right too much patient

involvement

Comments 
4. Were you able to talk about everything you wanted to in the group session?

$$
\text { Yes No }
$$

Comments

5. Did the group sessions provide you with enough information for you to now manage your symptoms without further advice?
Yes
No

Comments

6. Now that you have been to this session, if you were to get dietary advice again what sort of session would you prefer (please circle)? group session one-to-one appointment either Comments:

Any additional comments? 


\section{Thank you for completing our survey.}

